Supplement of Clim. Past, 16, 1043-1059, 2020

https://doi.org/10.5194/cp-16-1043-2020-supplement

(c) Author(s) 2020. This work is distributed under

the Creative Commons Attribution 4.0 License.

(c) (1)

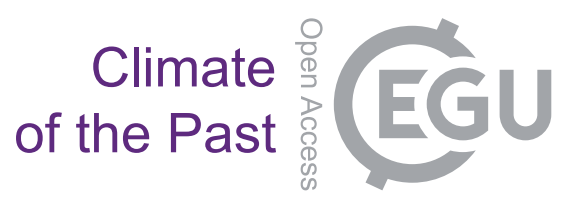

Supplement of

\title{
Application and evaluation of the dendroclimatic process-based model MAIDEN during the last century in Canada and Europe
}

\section{Jeanne Rezsöhazy et al.}

Correspondence to: Jeanne Rezsöhazy (jeanne.rezsohazy@uclouvain.be)

The copyright of individual parts of the supplement might differ from the CC BY 4.0 License. 


\section{S1: Supplementary materials}

Table S1. Main constants linked to site conditions and control parameters in the MAIDEN model.

\begin{tabular}{l|l|l}
\hline Parameter & Meaning & Units \\
\hline exp_site & $\begin{array}{l}\text { Indicates if the species at the } \\
\text { site is a deciduous (1) or ev- } \\
\text { ergreen (2) tree }\end{array}$ & no unit (1 or 2) \\
& $\begin{array}{l}\text { Station elevation } \\
\text { base_elev_cst }\end{array}$ & meters \\
base_isoh_cst & Station isohyet & centimeters \\
site_lat_cst & Site latitude & degrees \\
site_elev_cst & Site elevation & meters \\
site_slp_cst & Site slope & degrees \\
site_asp_cst & Site aspect & degrees \\
site_isoh_cst & Site isohyet & centimeters \\
site_ehoriz_cst & Site East slope & degrees \\
site_whoriz_cst & Site West slope & degrees \\
thick1-2-3 or 4 & Soil layer thickness & meters \\
finefracl-2-3 or 4 & $\%$ of fine roots in the soil & Coeff. between 0-1 \\
layer & $\%$ of clay in the soil layer & $\%$ \\
clayl-2-3 or 4 & $\%$ of sand in the soil layer & $\%$ \\
sand1-2-3 or 4 &
\end{tabular}

Table S2. GHCN (Table 2) stations used for daily climate data at the European sites (Fig. 2).

\begin{tabular}{rlccc}
\hline Site & Time period & Station name & Station lat/lon & Station elevation \\
\hline \multirow{2}{*}{ FINL } & $1900-1944 / 1950-2000$ & Sodankyla & $67.37 \mathrm{~N} 26.65 \mathrm{E}$ & $179 \mathrm{~m}$ \\
EALP & $1950-2000$ & Zugspitze & $47.42 \mathrm{~N} 10.99 \mathrm{E}$ & $2964 \mathrm{~m}$ \\
& $1910-1949$ & Innsbruck & $47.27 \mathrm{~N} 11.4 \mathrm{E}$ & $577 \mathrm{~m}$ \\
SWIT179 & $1910-2000$ & Saentis & $47.25 \mathrm{~N} 9.35 \mathrm{E}$ & $2502 \mathrm{~m}$ \\
\hline
\end{tabular}


Table S3. Calibrated parameters of the MAIDEN model (Gennaretti et al., 2017).

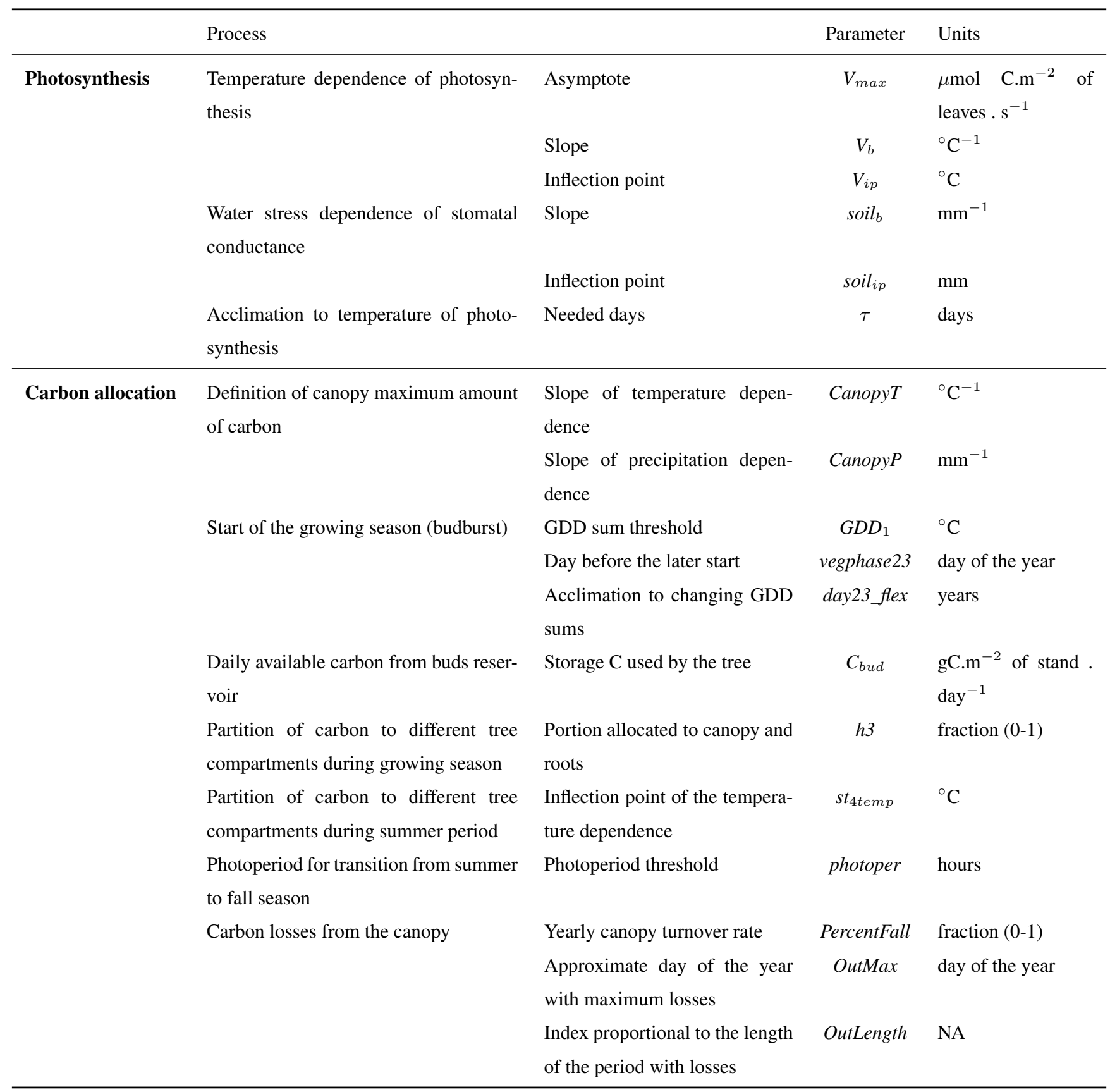


Table S4. MAIDEN calibrated parameters values (Table S3) over the 1950-2000 period for the twenty-one Eastern Canadian taiga sites, five aggregated Eastern Canadian taiga sites (NRCAN (5') climatic dataset, Fig. 1, Table 2) and three European sites (GHCN station data, Fig. 2, Table 2).

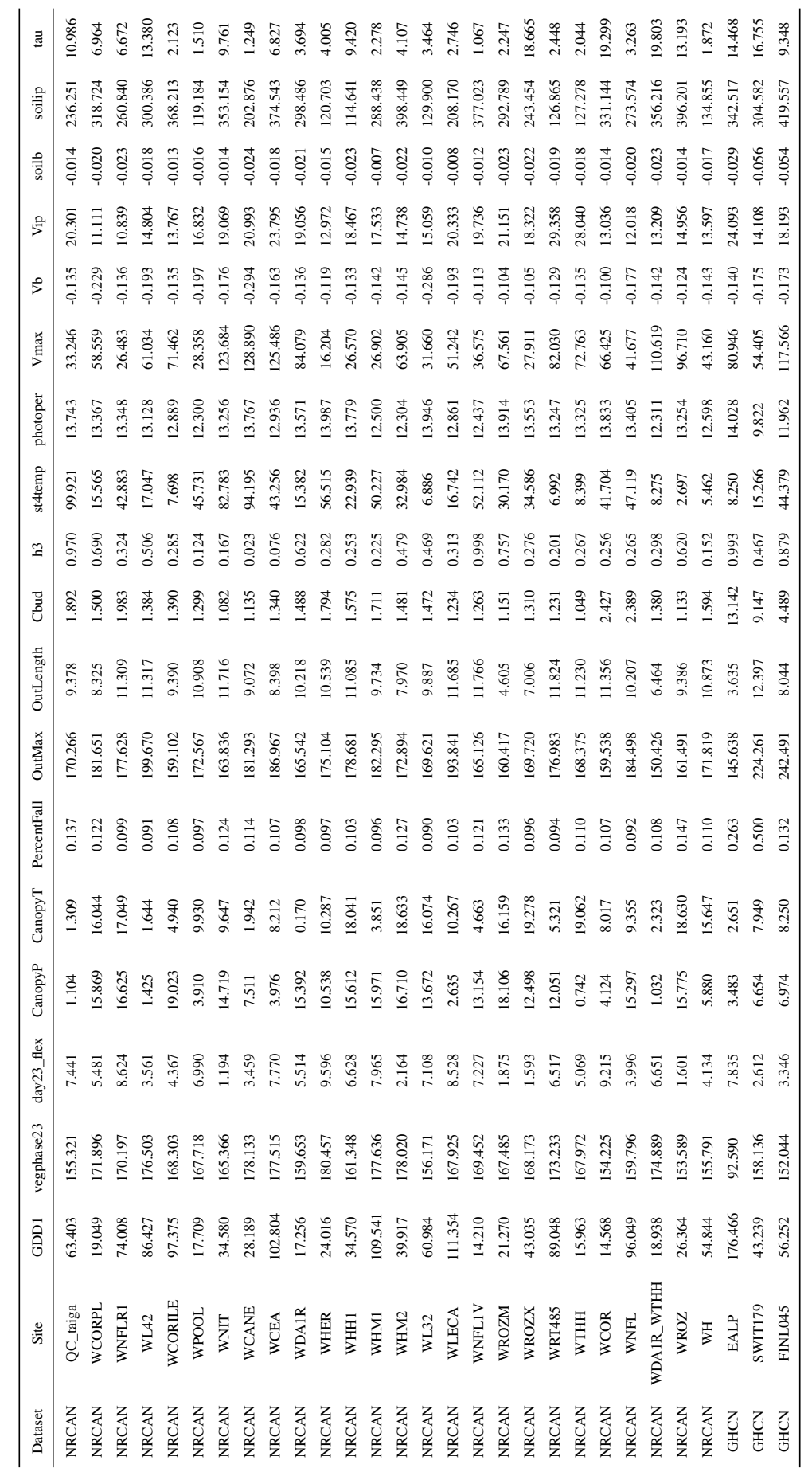


Table S5. MAIDEN calibrated parameters values (Table S3) over the 1950-2000 period for the twenty-one Eastern Canadian taiga sites and five aggregated Eastern Canadian taiga sites $\left(\operatorname{GMF}\left(1^{\circ}\right)\right.$ climatic dataset, Fig. 1, Table 2).

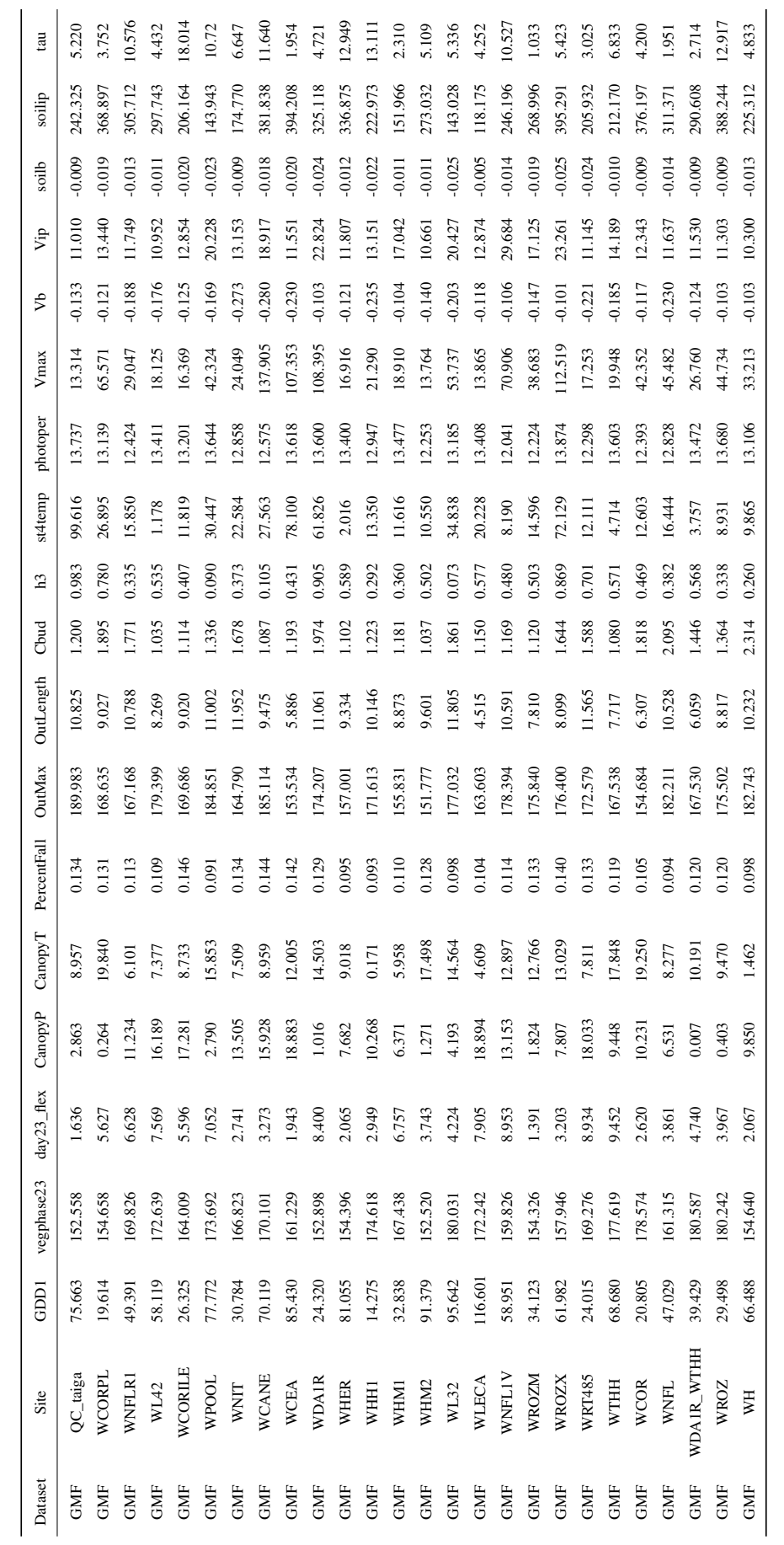


Table S6. MAIDEN calibrated parameters values (Table S3) over the 1950-2000 period for the twenty-one Eastern Canadian taiga sites and five aggregated Eastern Canadian taiga sites (20CRv2c corr. $\left(2^{\circ}\right)$ climatic dataset, Fig. 1, Table 2).

\begin{tabular}{|c|c|}
\hline & 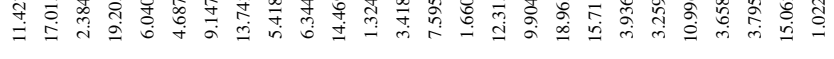 \\
\hline & 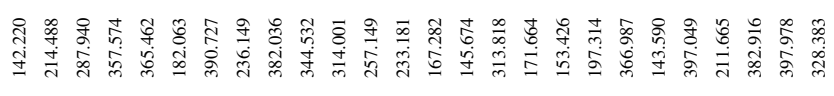 \\
\hline$\overline{\bar{\sigma}}$ & 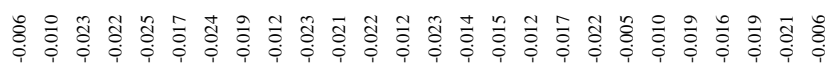 \\
\hline & 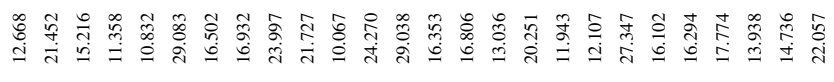 \\
\hline 8 & 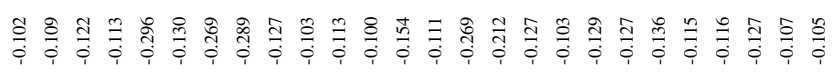 \\
\hline 尊 & 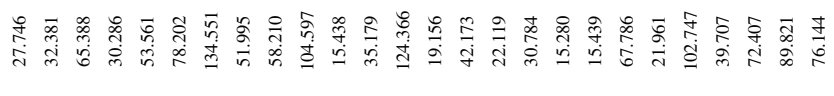 \\
\hline & 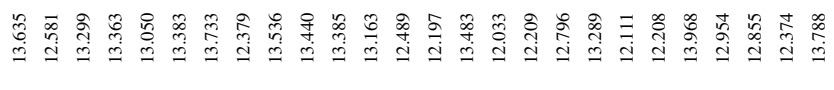 \\
\hline 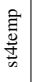 & 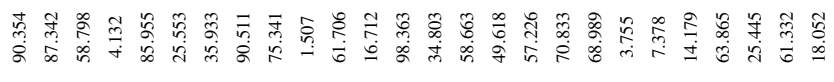 \\
\hline$\cong$ & \\
\hline 言 & $\stackrel{-1}{7}$ \\
\hline & \\
\hline 諝 & 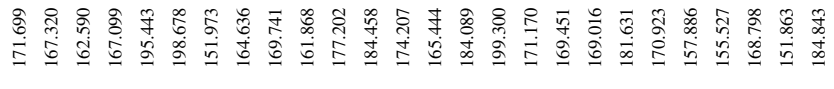 \\
\hline & 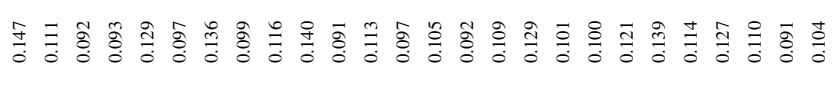 \\
\hline 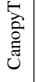 & 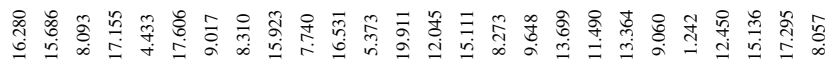 \\
\hline & 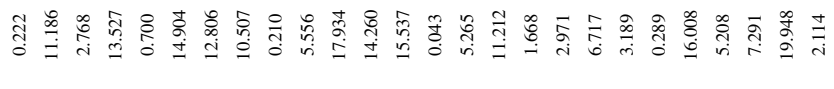 \\
\hline & 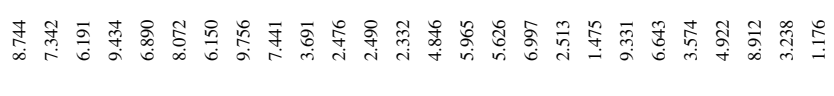 \\
\hline 美 & 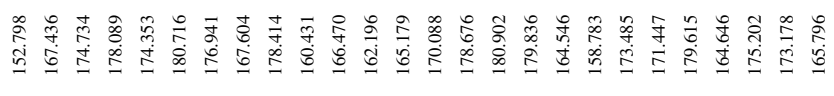 \\
\hline 5 & 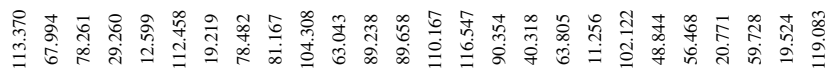 \\
\hline & 号居点 \\
\hline & \\
\hline & 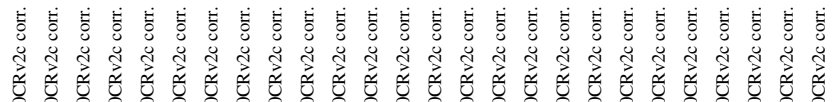 \\
\hline
\end{tabular}


Table S7. MAIDEN calibrated parameters values (Table S3) over the 1950-2000 period for the twenty-one Eastern Canadian taiga sites and five aggregated Eastern Canadian taiga sites (20CRv2c $\left(2^{\circ}\right)$ climatic dataset, Fig. 1, Table 2).

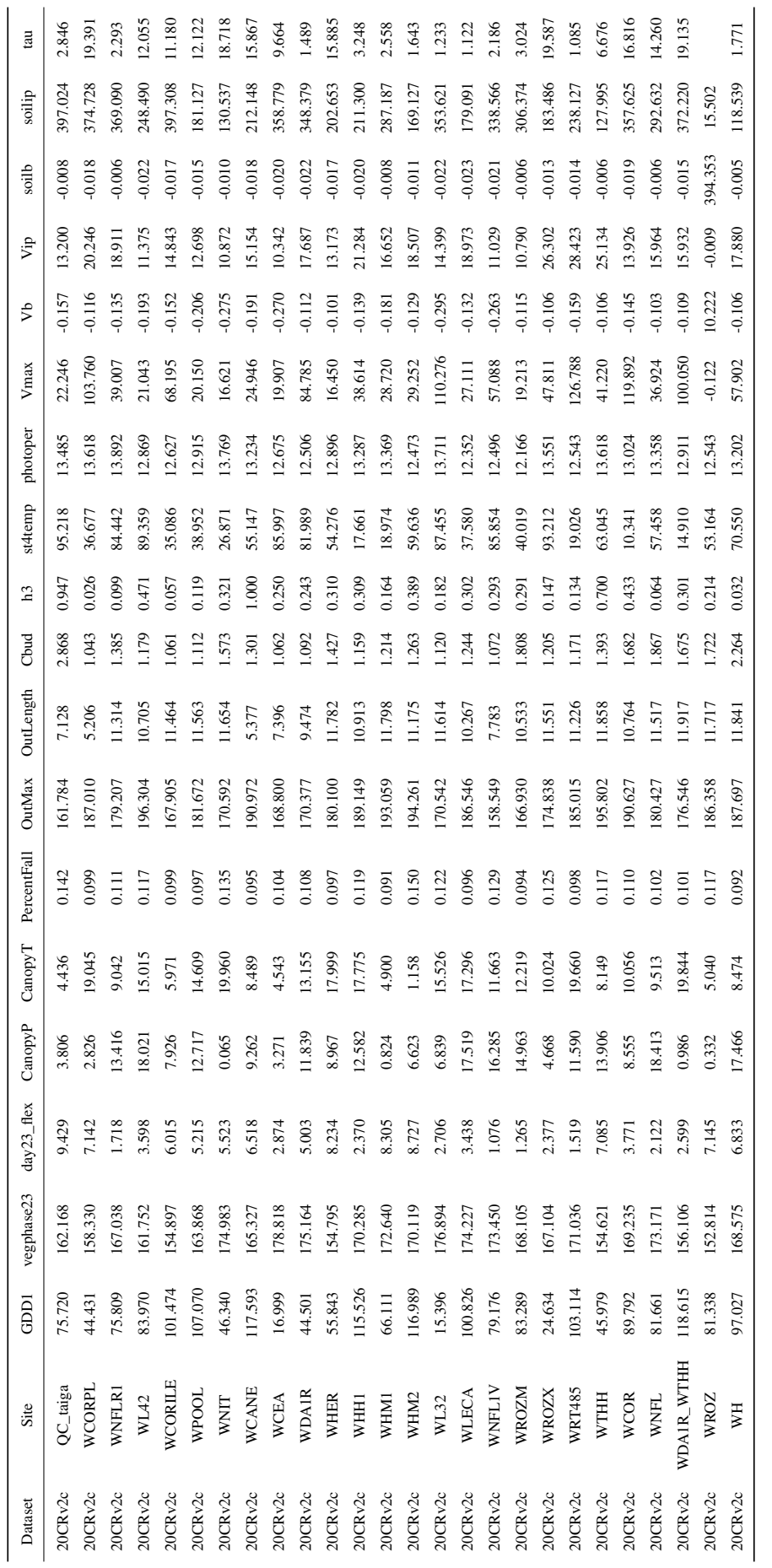


Table S8. VS-Lite calibrated parameters values (Sect. 2.3.2) over the 1950-2000 period for the twenty-one Eastern Canadian taiga sites (NRCAN (5') climatic dataset, Fig. 1a, Table 2) and three European sites (GHCN station data, Fig. 2, Table 2).

\begin{tabular}{lccccc}
\hline Dataset & Sites & T1 & T2 & M1 & M2 \\
& & & & & \\
\hline NRCAN & QC_taiga & 2.430 & 15.727 & 0.053 & 0.429 \\
NRCAN & WCORPL & 4.612 & 12.497 & 0.035 & 0.275 \\
NRCAN & WNFLR1 & 4.914 & 11.493 & 0.033 & 0.357 \\
NRCAN & WL42 & 7.259 & 11.658 & 0.070 & 0.457 \\
NRCAN & WCORILE & 3.058 & 12.002 & 0.032 & 0.194 \\
NRCAN & WPOOL & 7.899 & 11.514 & 0.066 & 0.194 \\
NRCAN & WNIT & 7.876 & 12.118 & 0.016 & 0.230 \\
NRCAN & WCANE & 7.264 & 11.557 & 0.077 & 0.171 \\
NRCAN & WCEA & 5.745 & 12.363 & 0.074 & 0.443 \\
NRCAN & WDA1R & 1.316 & 14.399 & 0.053 & 0.183 \\
NRCAN & WHER & 2.795 & 19.393 & 0.058 & 0.258 \\
NRCAN & WHH1 & 7.490 & 11.677 & 0.007 & 0.190 \\
NRCAN & WHM1 & 7.660 & 12.939 & 0.017 & 0.220 \\
NRCAN & WHM2 & 8.843 & 12.165 & 0.040 & 0.168 \\
NRCAN & WL32 & 7.642 & 13.785 & 0.013 & 0.231 \\
NRCAN & WLECA & 8.389 & 12.148 & 0.032 & 0.169 \\
NRCAN & WNFL1V & 3.575 & 11.542 & 0.086 & 0.465 \\
NRCAN & WROZM & 1.726 & 11.656 & 0.027 & 0.153 \\
NRCAN & WROZX & 6.170 & 11.382 & 0.070 & 0.473 \\
NRCAN & WRT485 & 2.014 & 17.012 & 0.001 & 0.158 \\
NRCAN & WTHH & 3.996 & 13.065 & 0.020 & 0.119 \\
GHCN & EALP & 8.242 & 22.117 & 0.058 & 0.277 \\
GHCN & SWIT179 & 1.480 & 21.912 & 0.052 & 0.294 \\
GHCN & FINL045 & 2.517 & 19.159 & 0.007 & 0.120 \\
\hline & & & & &
\end{tabular}


Table S9. VS-Lite calibrated parameters values (Sect. 2.3.2) over the 1950-2000 period for the twenty-one Eastern Canadian taiga sites $\left(\operatorname{GMF}\left(1^{\circ}\right)\right.$ climatic dataset, Fig. 1a, Table 2).

\begin{tabular}{cccccc}
\hline Dataset & Sites & T1 & T2 & M1 & M2 \\
& & & & & \\
\hline GMF & QC_taiga & 7.934 & 20.259 & 0.036 & 0.210 \\
GMF & WCORPL & 2.574 & 12.366 & 0.027 & 0.233 \\
GMF & WNFLR1 & 3.124 & 10.795 & 0.018 & 0.404 \\
GMF & WL42 & 6.973 & 10.861 & 0.036 & 0.378 \\
GMF & WCORILE & 2.585 & 12.279 & 0.025 & 0.132 \\
GMF & WPOOL & 8.036 & 11.556 & 0.042 & 0.266 \\
GMF & WNIT & 8.193 & 13.365 & 0.028 & 0.219 \\
GMF & WCANE & 7.517 & 12.862 & 0.089 & 0.482 \\
GMF & WCEA & 6.072 & 11.476 & 0.080 & 0.469 \\
GMF & WDA1R & 1.613 & 22.429 & 0.003 & 0.318 \\
GMF & WHER & 4.808 & 12.558 & 0.040 & 0.439 \\
GMF & WHH1 & 7.303 & 11.754 & 0.061 & 0.259 \\
GMF & WHM1 & 2.750 & 13.427 & 0.009 & 0.223 \\
GMF & WHM2 & 5.479 & 12.363 & 0.023 & 0.185 \\
GMF & WL32 & 8.300 & 15.367 & 0.007 & 0.355 \\
GMF & WLECA & 7.638 & 11.770 & 0.017 & 0.464 \\
GMF & WNFL1V & 3.241 & 11.483 & 0.080 & 0.468 \\
GMF & WROZM & 1.867 & 15.193 & 0.060 & 0.386 \\
GMF & WROZX & 1.470 & 14.070 & 0.055 & 0.154 \\
GMF & WRT485 & 1.141 & 17.046 & 0.075 & 0.386 \\
GMF & WTHH & 3.033 & 13.675 & 0.012 & 0.138 \\
\hline & & & & &
\end{tabular}


Table S10. VS-Lite calibrated parameters values (Sect. 2.3.2) over the 1950-2000 period for the twenty-one Eastern Canadian taiga sites (20CRv2c corr. $\left(2^{\circ}\right)$ climatic dataset, Fig. 1a, Table 2$)$.

\begin{tabular}{|c|c|c|c|c|c|}
\hline Dataset & Sites & $\mathrm{T} 1$ & $\mathrm{~T} 2$ & M1 & M2 \\
\hline 20CRv2c corr. & QC_taiga & 7.000 & 14.214 & 0.094 & 0.436 \\
\hline 20CRv2c corr. & WCORPL & 1.996 & 11.968 & 0.043 & 0.276 \\
\hline 20CRv2c corr. & WNFLR1 & 2.443 & 19.159 & 0.011 & 0.246 \\
\hline 20CRv2c corr. & WL42 & 7.672 & 11.259 & 0.080 & 0.447 \\
\hline 20CRv2c corr. & WCORILE & 3.102 & 12.325 & 0.056 & 0.254 \\
\hline 20CRv2c corr. & WPOOL & 6.812 & 10.631 & 0.005 & 0.221 \\
\hline 20CRv2c corr. & WNIT & 8.347 & 12.275 & 0.055 & 0.201 \\
\hline 20CRv2c corr. & WCANE & 8.277 & 12.194 & 0.017 & 0.200 \\
\hline 20CRv2c corr. & WCEA & 2.681 & 12.493 & 0.043 & 0.410 \\
\hline 20CRv2c corr. & WDA1R & 3.382 & 18.603 & 0.013 & 0.295 \\
\hline 20CRv2c corr. & WHER & 4.768 & 12.783 & 0.027 & 0.196 \\
\hline 20CRv2c corr. & WHH1 & 7.464 & 11.322 & 0.058 & 0.116 \\
\hline 20CRv2c corr. & WHM1 & 8.472 & 15.277 & 0.082 & 0.258 \\
\hline 20CRv2c corr. & WHM2 & 8.383 & 18.934 & 0.053 & 0.218 \\
\hline 20CRv2c corr. & WL32 & 8.446 & 14.245 & 0.011 & 0.108 \\
\hline 20CRv2c corr. & WLECA & 7.556 & 13.389 & 0.023 & 0.446 \\
\hline 20CRv2c corr. & WNFL1V & 3.803 & 15.342 & 0.011 & 0.168 \\
\hline 20CRv2c corr. & WROZM & 8.262 & 14.324 & 0.001 & 0.256 \\
\hline 20CRv2c corr. & WROZX & 8.633 & 14.984 & 0.017 & 0.262 \\
\hline 20CRv2c corr. & WRT485 & 8.381 & 15.478 & 0.016 & 0.189 \\
\hline 20CRv2c corr. & WTHH & 3.802 & 15.778 & 0.033 & 0.105 \\
\hline
\end{tabular}


Table S11. VS-Lite calibrated parameters values (Sect. 2.3.2) over the 1950-2000 period for the twenty-one Eastern Canadian taiga sites (20CRv2c $\left(2^{\circ}\right)$ climatic dataset, Fig. 1a, Table 2).

\begin{tabular}{lccccc}
\hline Dataset & Sites & T1 & T2 & M1 & M2 \\
& & & & & \\
\hline 20CRv2c & QC_taiga & 8.378 & 13.382 & 0.036 & 0.319 \\
20CRv2c & WCORPL & 7.532 & 18.410 & 0.036 & 0.270 \\
20CRv2c & WNFLR1 & 8.399 & 19.795 & 0.014 & 0.110 \\
20CRv2c & WL42 & 6.012 & 10.591 & 0.031 & 0.314 \\
20CRv2c & WCORILE & 7.629 & 10.677 & 0.047 & 0.262 \\
20CRv2c & WPOOL & 7.219 & 10.537 & 0.076 & 0.281 \\
20CRv2c & WNIT & 7.990 & 12.538 & 0.035 & 0.267 \\
20CRv2c & WCANE & 7.118 & 10.445 & 0.015 & 0.279 \\
20CRv2c & WCEA & 5.313 & 15.658 & 0.019 & 0.238 \\
20CRv2c & WDA1R & 8.167 & 19.349 & 0.088 & 0.194 \\
20CRv2c & WHER & 3.440 & 17.681 & 0.062 & 0.366 \\
20CRv2c & WHH1 & 6.951 & 19.205 & 0.051 & 0.366 \\
20CRv2c & WHM1 & 7.395 & 22.139 & 0.031 & 0.266 \\
20CRv2c & WHM2 & 7.551 & 18.823 & 0.024 & 0.212 \\
20CRv2c & WL32 & 8.308 & 14.045 & 0.008 & 0.234 \\
20CRv2c & WLECA & 6.798 & 14.509 & 0.050 & 0.391 \\
20CRv2c & WNFL1V & 8.604 & 15.787 & 0.042 & 0.153 \\
20CRv2c & WROZM & 8.131 & 12.693 & 0.060 & 0.133 \\
20CRv2c & WROZX & 8.645 & 16.846 & 0.035 & 0.205 \\
20CRv2c & WRT485 & 7.555 & 20.034 & 0.019 & 0.210 \\
20CRv2c & WTHH & 6.906 & 20.691 & 0.014 & 0.240 \\
\hline
\end{tabular}




\section{Carbon allocation parameters for QC_taiga}
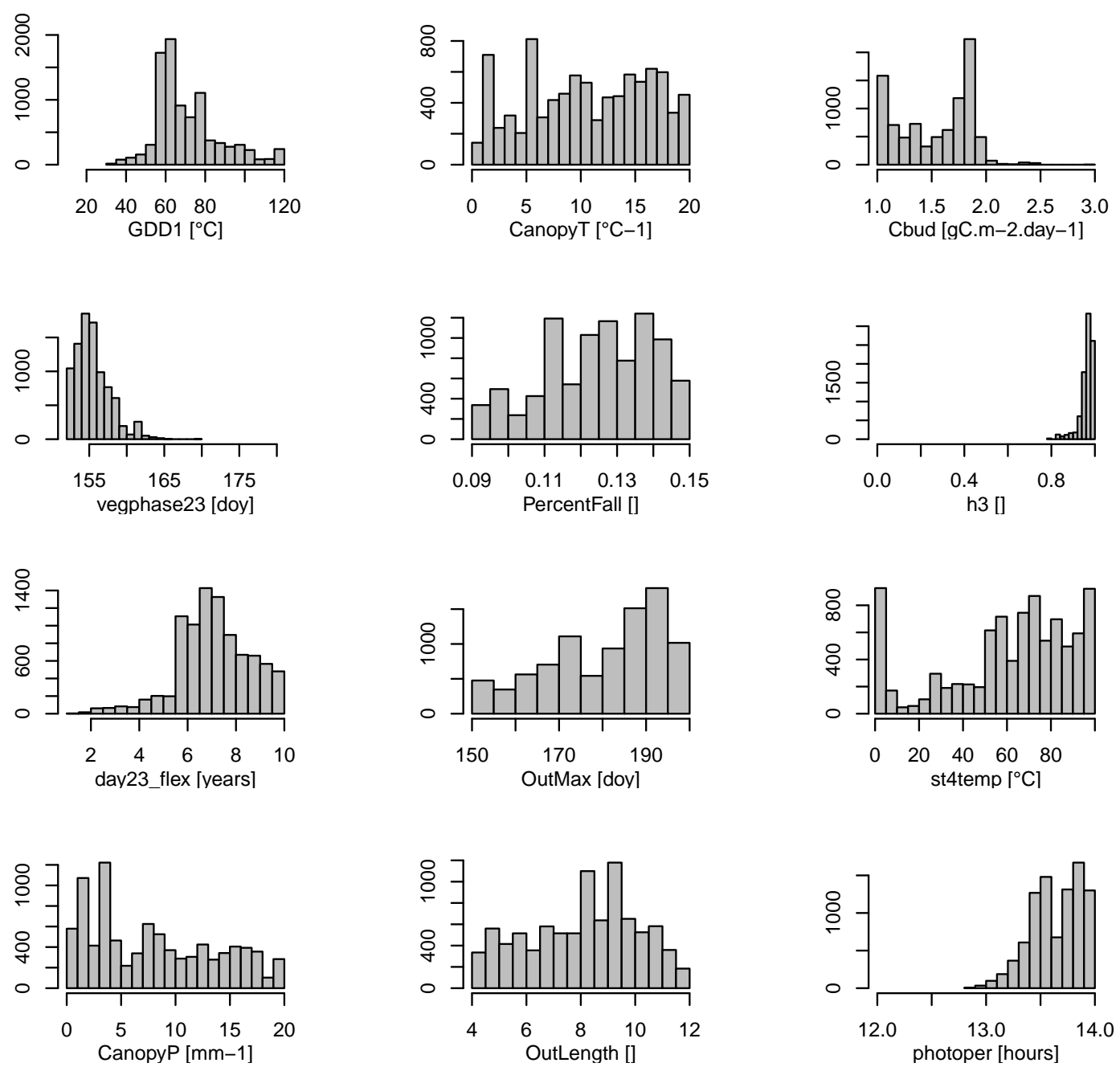

Figure S1. Posterior frequency distributions of carbon allocation parameters (Table S3) at QC_taiga site (NRCAN (5') climatic dataset) (Fig. 1a, Table 2) for the 1950-2000 calibration period. 


\section{Photosynthesis parameters for QC_taiga}
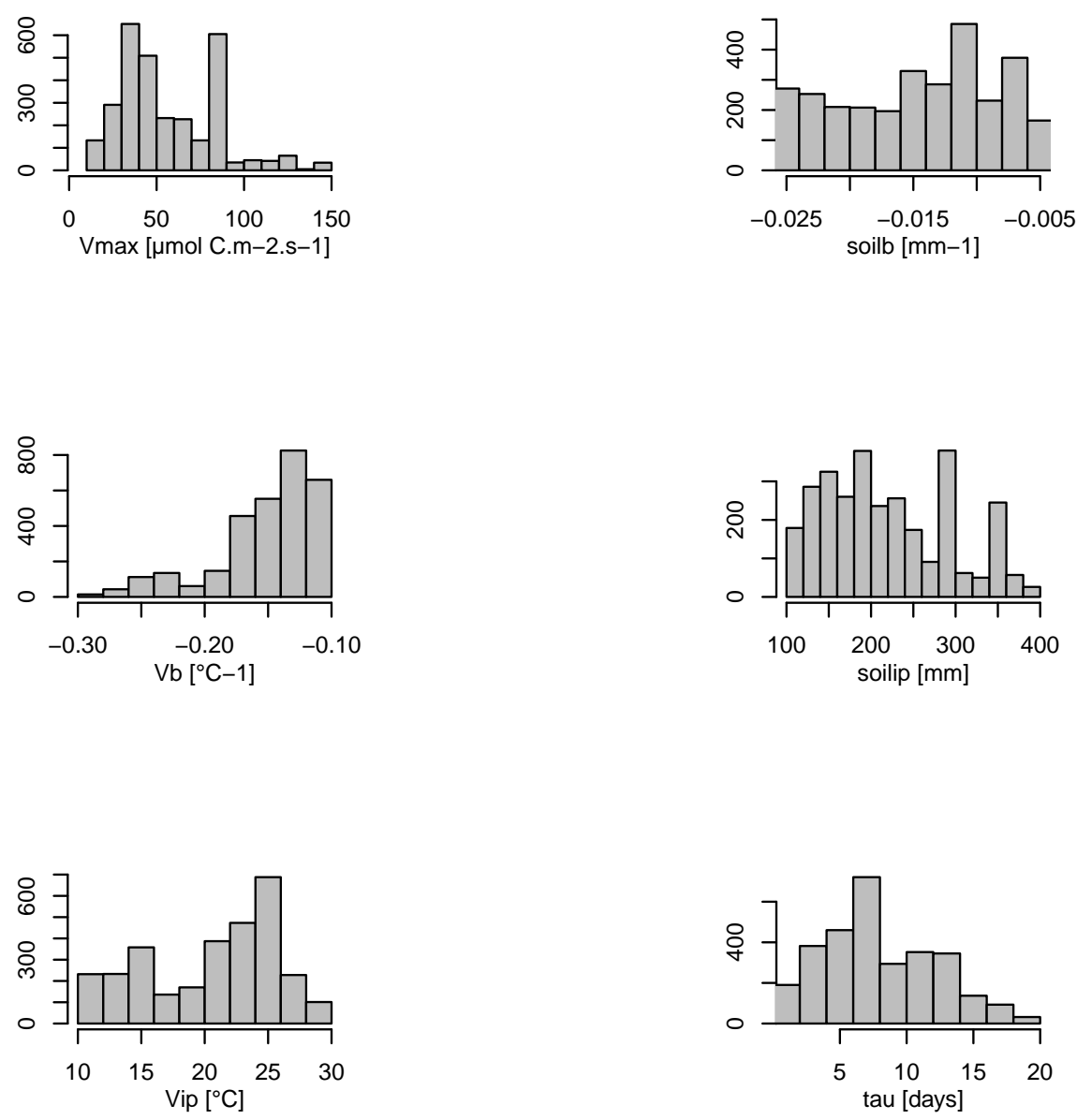

Figure S2. Posterior frequency distributions of photosynthesis parameters (Table S3) at QC_taiga site (NRCAN (5') climatic dataset) (Fig. 1a, Table 2) for the 1950-2000 calibration period. 


\section{Carbon allocation parameters for WCORPL}
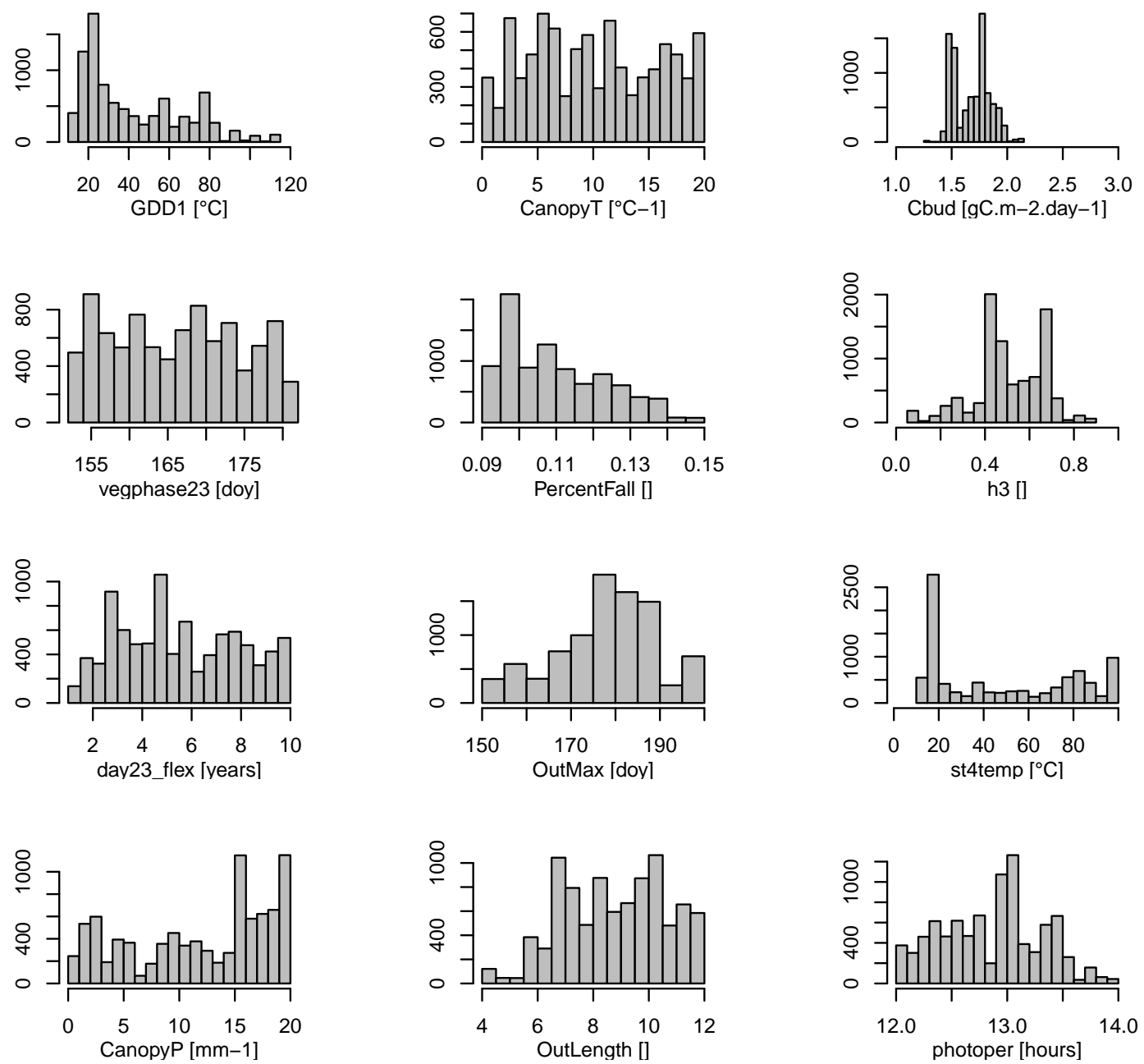

Figure S3. As in Fig. S1 at WCORPL site. 


\section{Photosynthesis parameters for WCORPL}
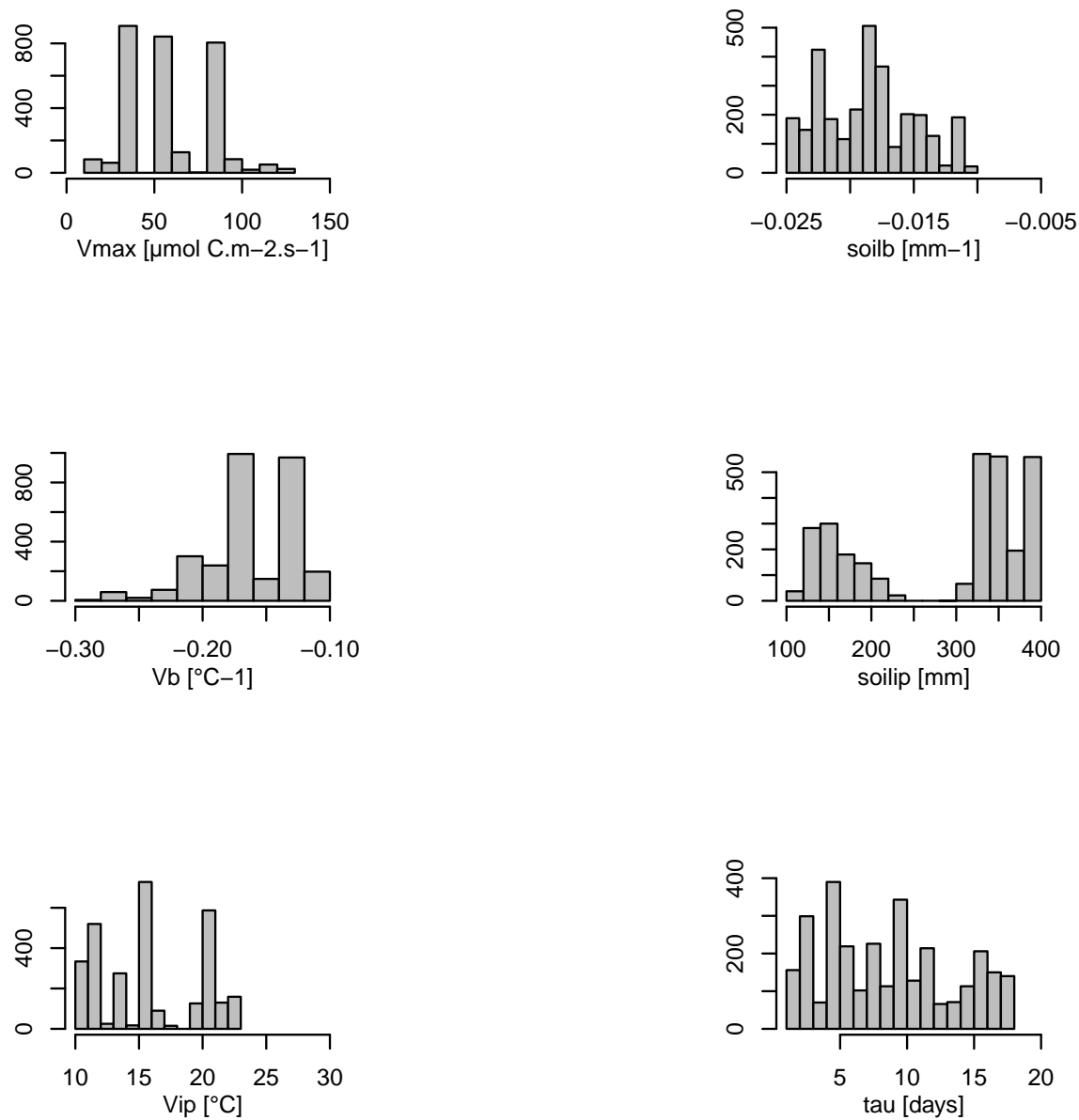

Figure S4. As in Fig. S2 at WCORPL site. 
Carbon allocation parameters for WCANE
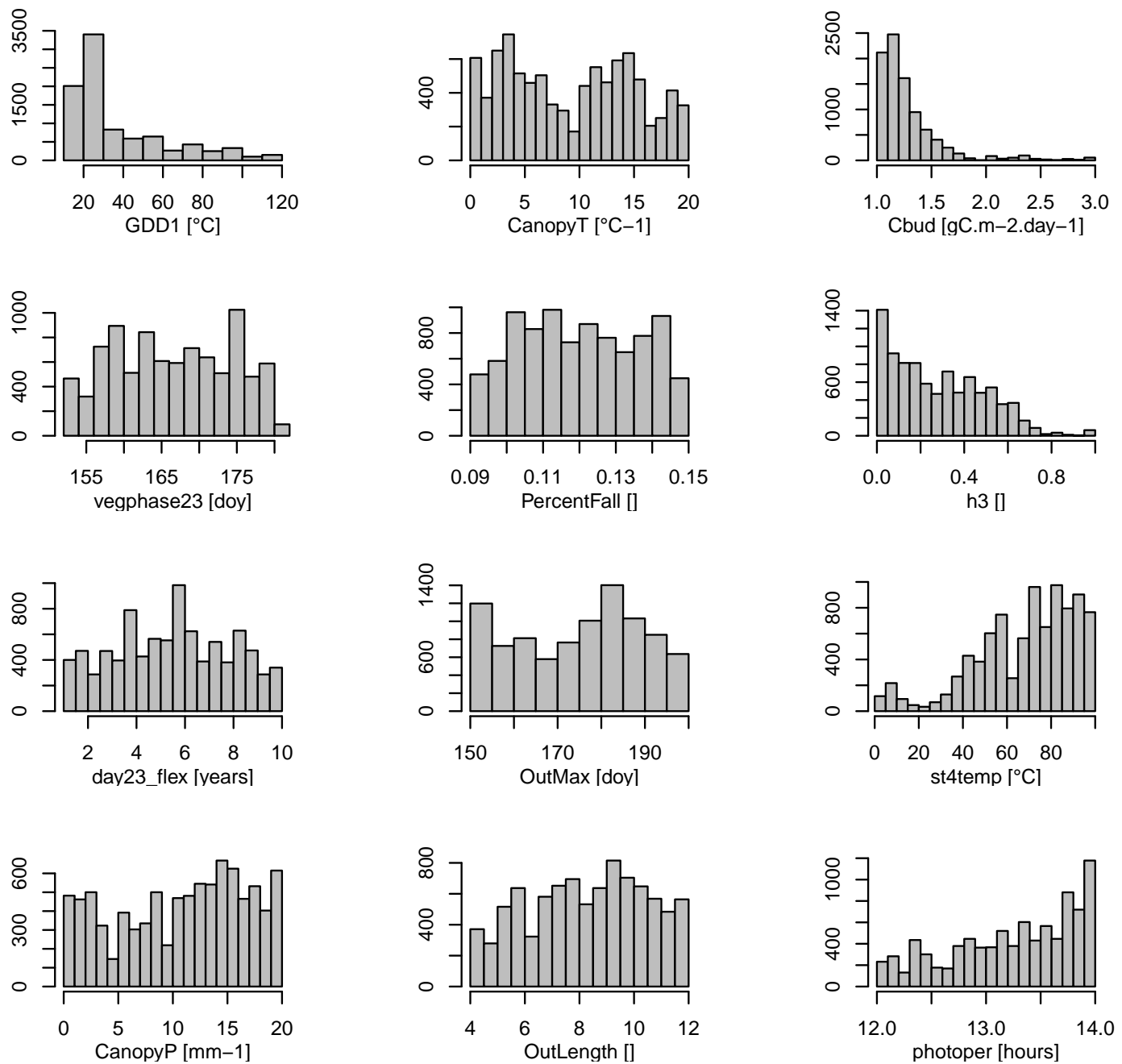

Figure S5. As in Fig. S1 at WCANE site. 

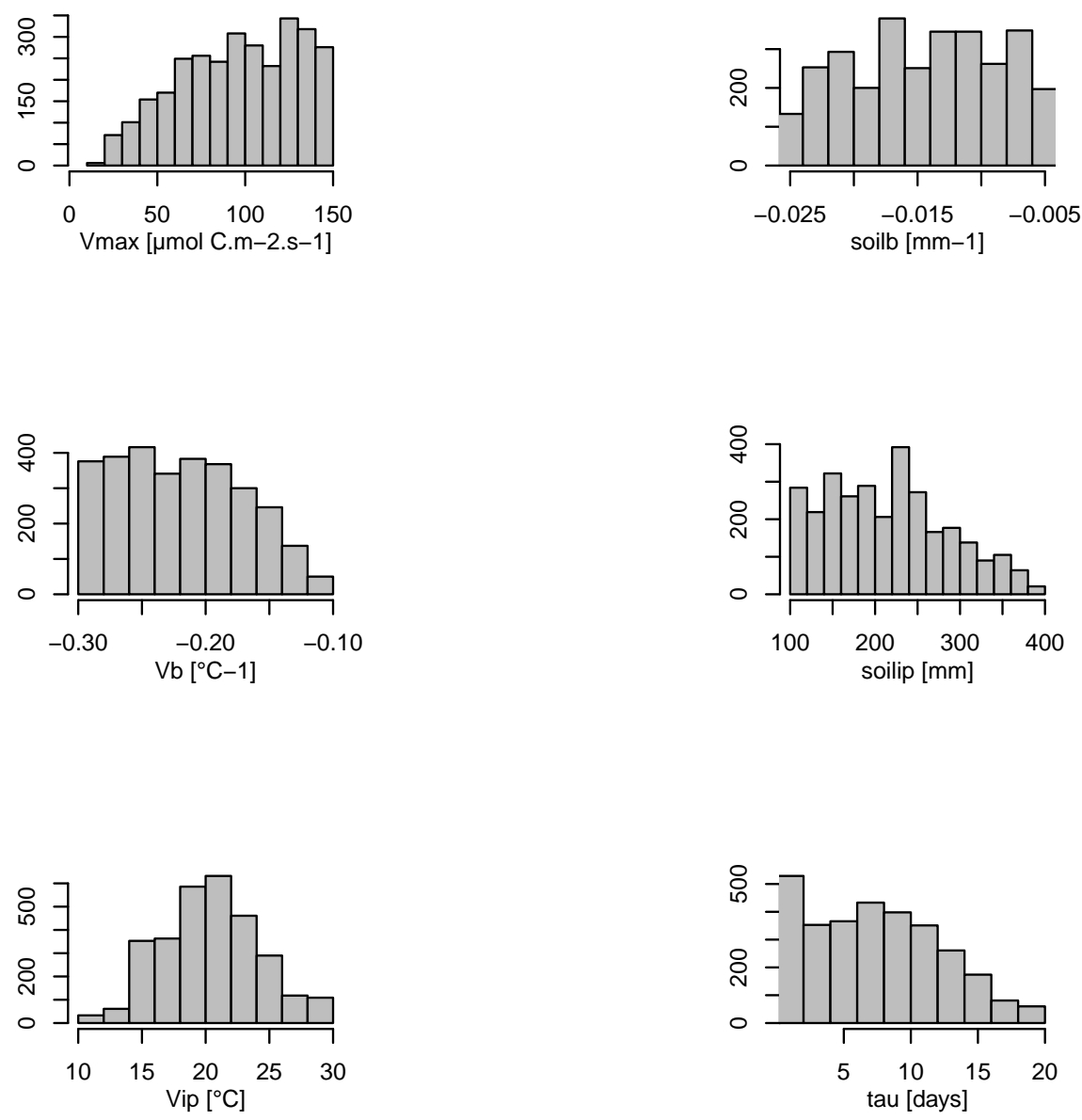

Figure S6. As in Fig. S2 at WCANE site. 


\section{Carbon allocation parameters for WCEA}
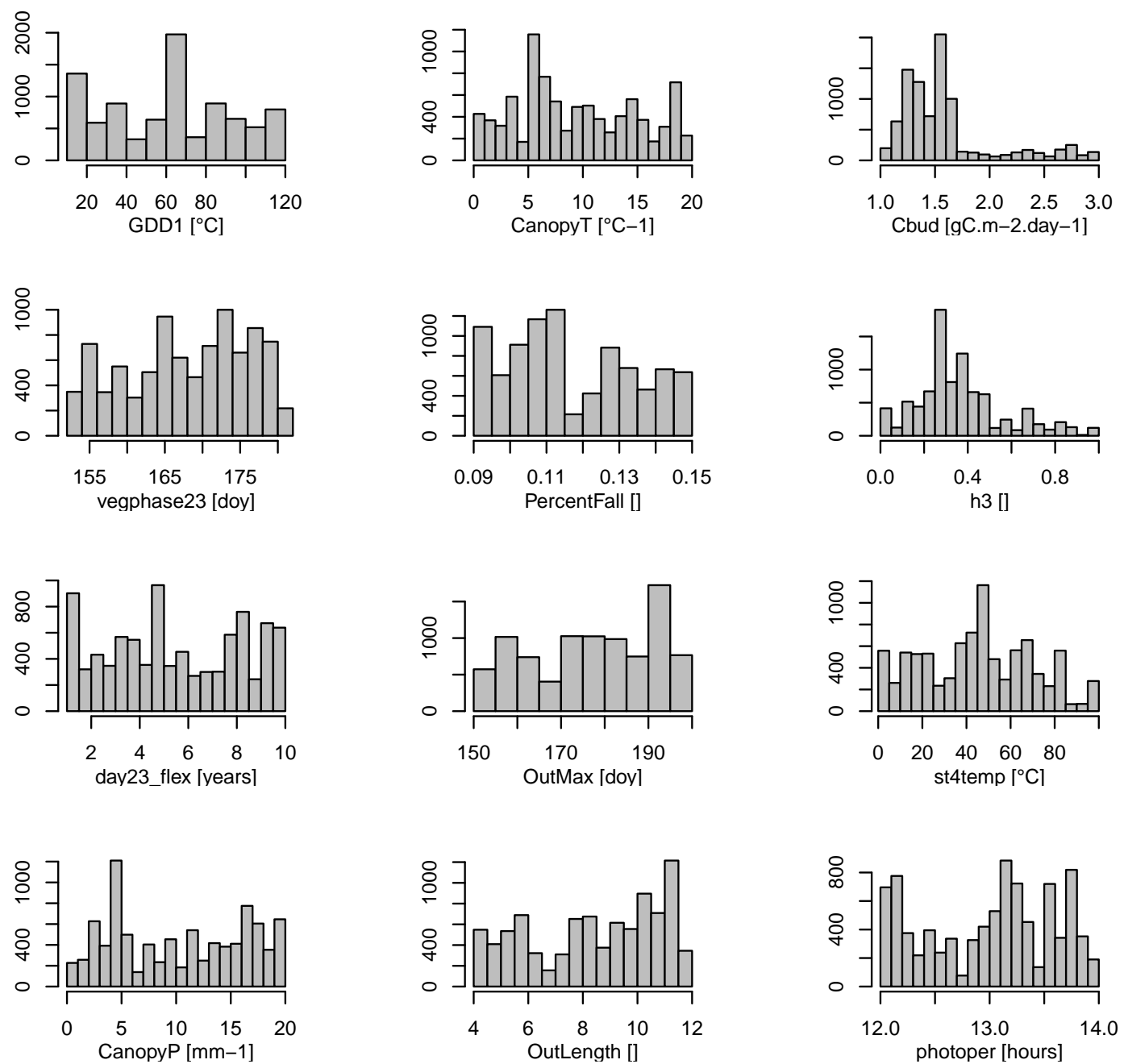

Figure S7. As in Fig. S1 at WCEA site. 


\section{Photosynthesis parameters for WCEA}
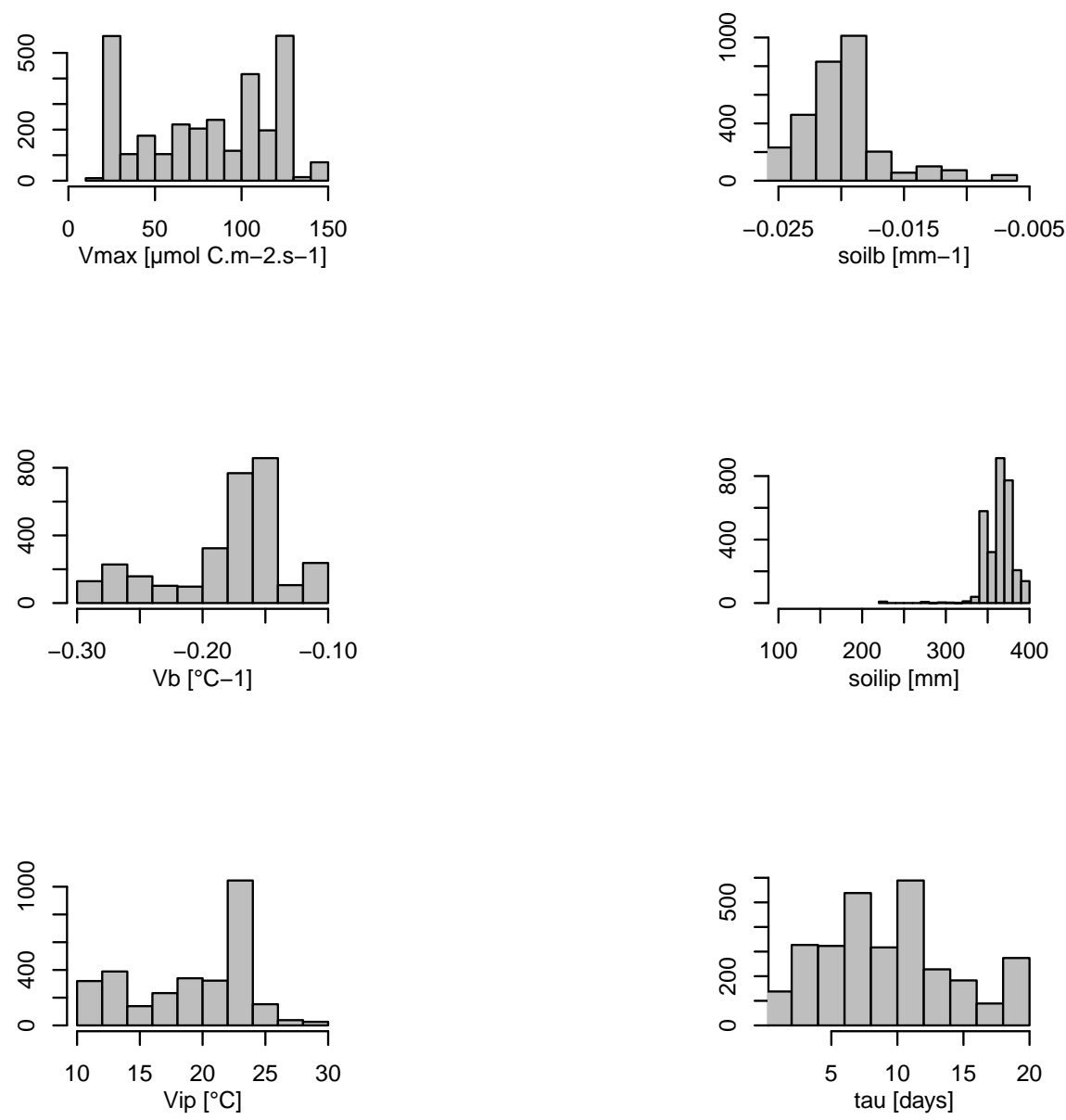

Figure S8. As in Fig. S2 at WCEA site. 
Carbon allocation parameters for WCORILE
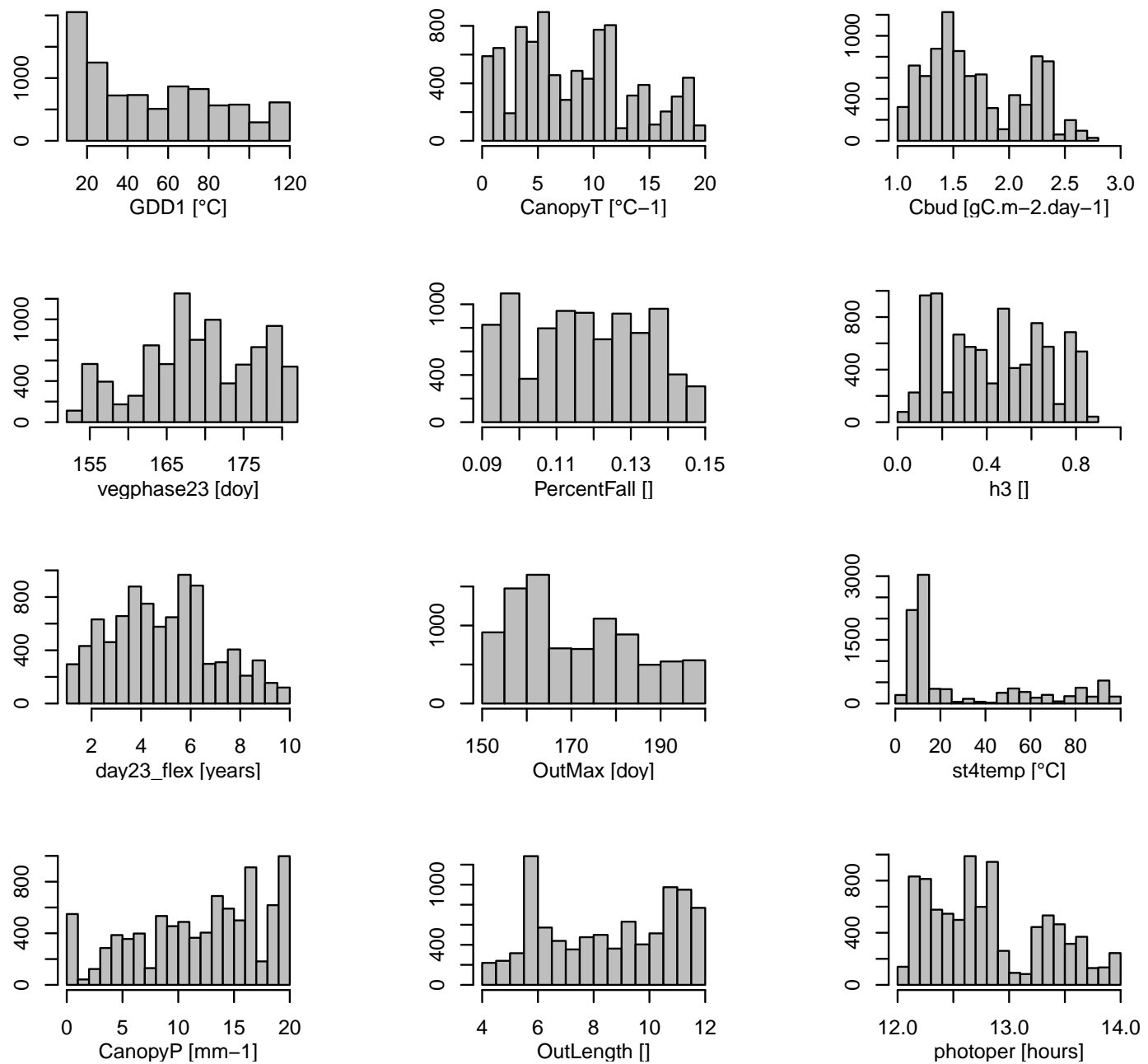

Figure S9. As in Fig. S1 at WCORILE site. 
Photosynthesis parameters for WCORILE
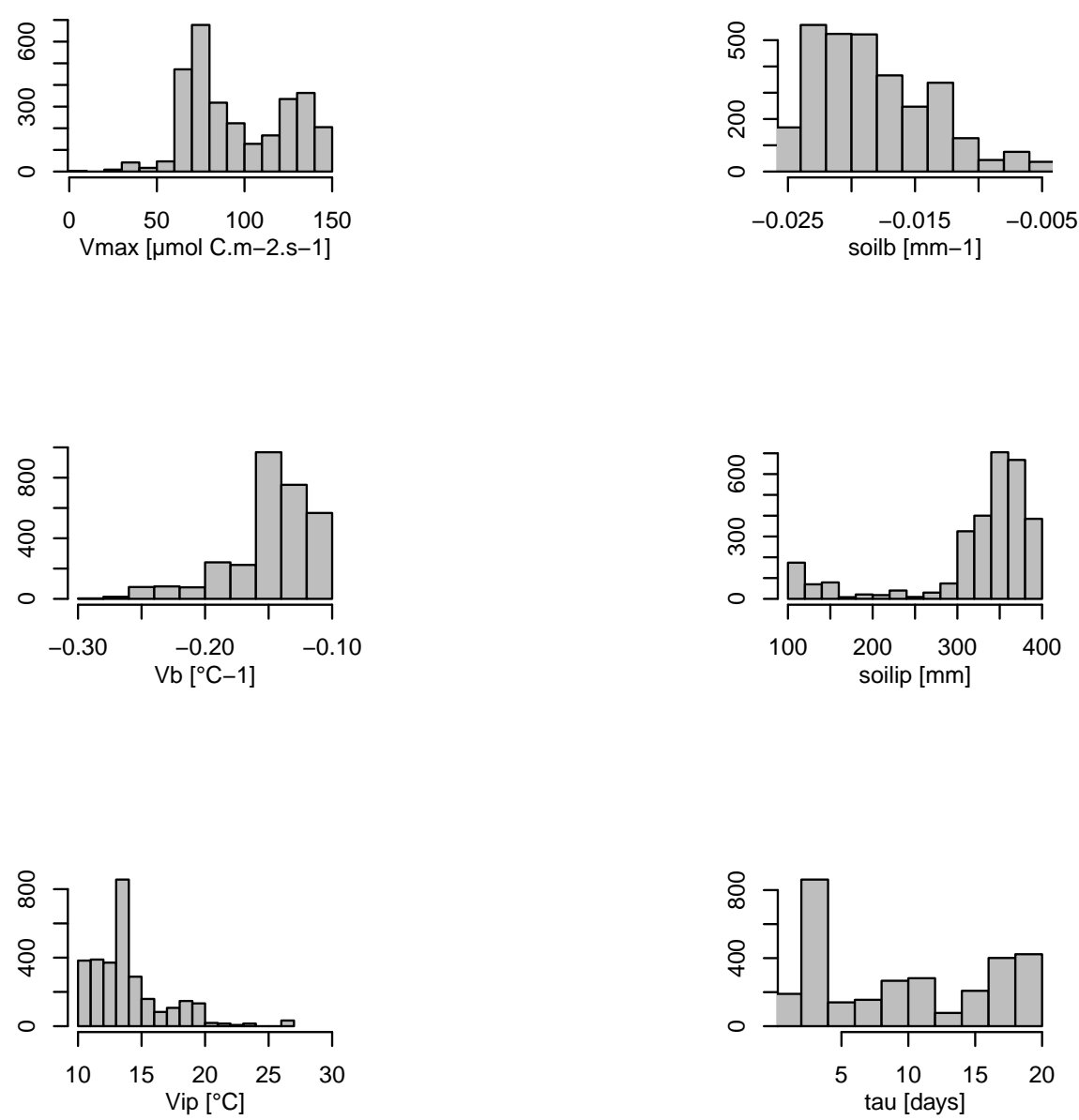

Figure S10. As in Fig. S2 at WCORILE site. 
Carbon allocation parameters for WDA1R
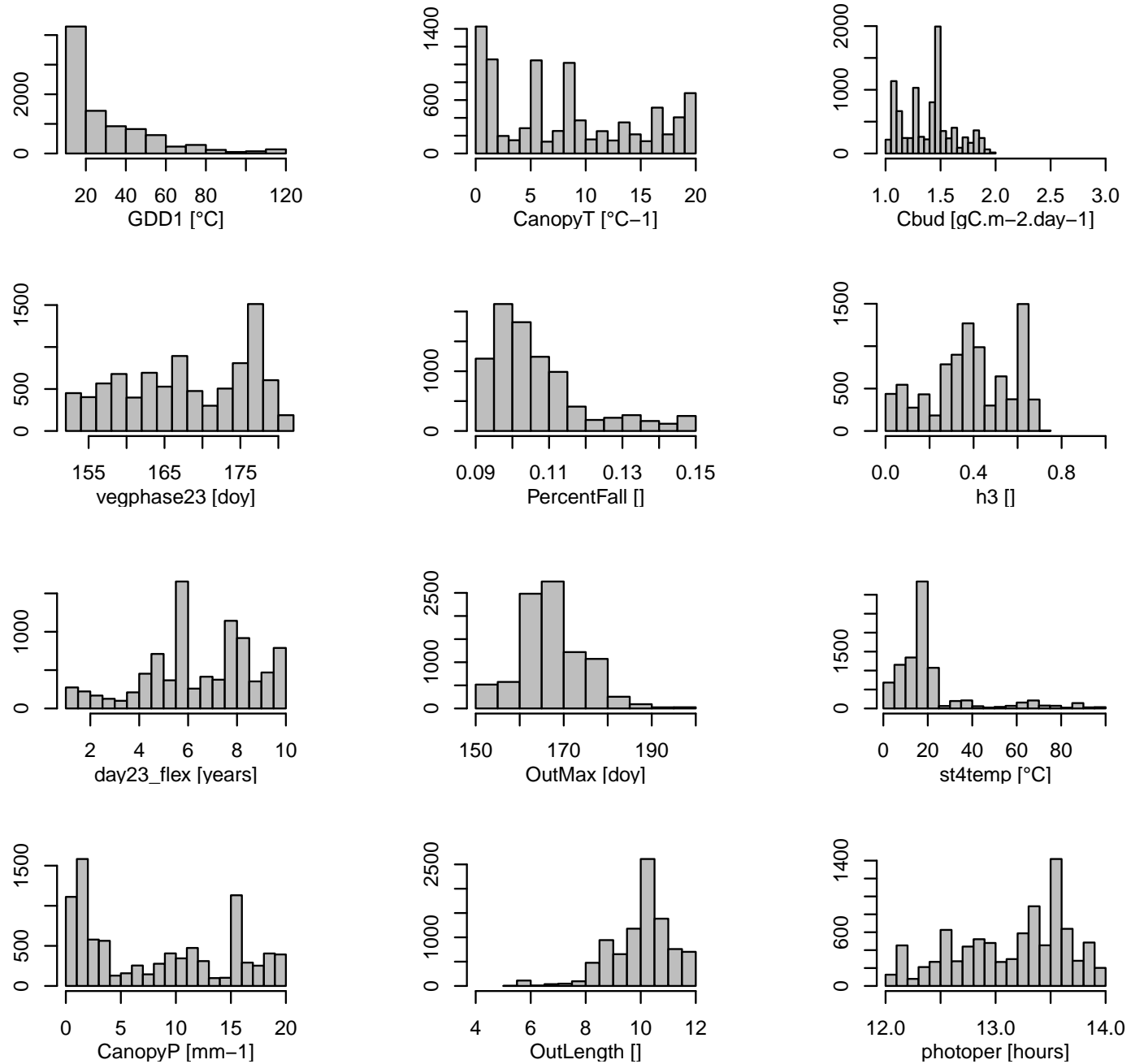

Figure S11. As in Fig. S1 at WDA1R site. 
Photosynthesis parameters for WDA1R
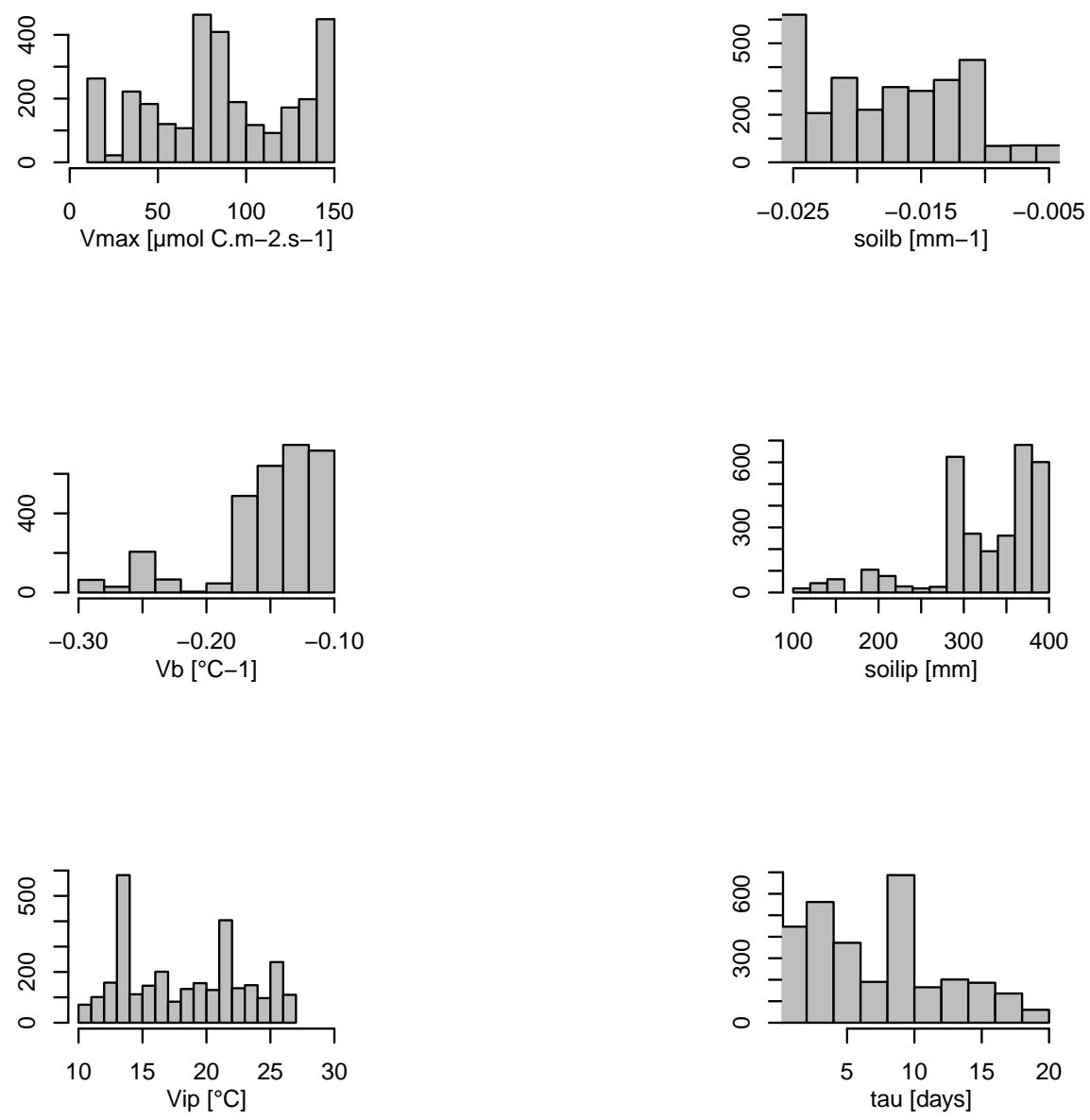

Figure S12. As in Fig. S2 at WDA1R site. 


\section{Carbon allocation parameters for WHER}
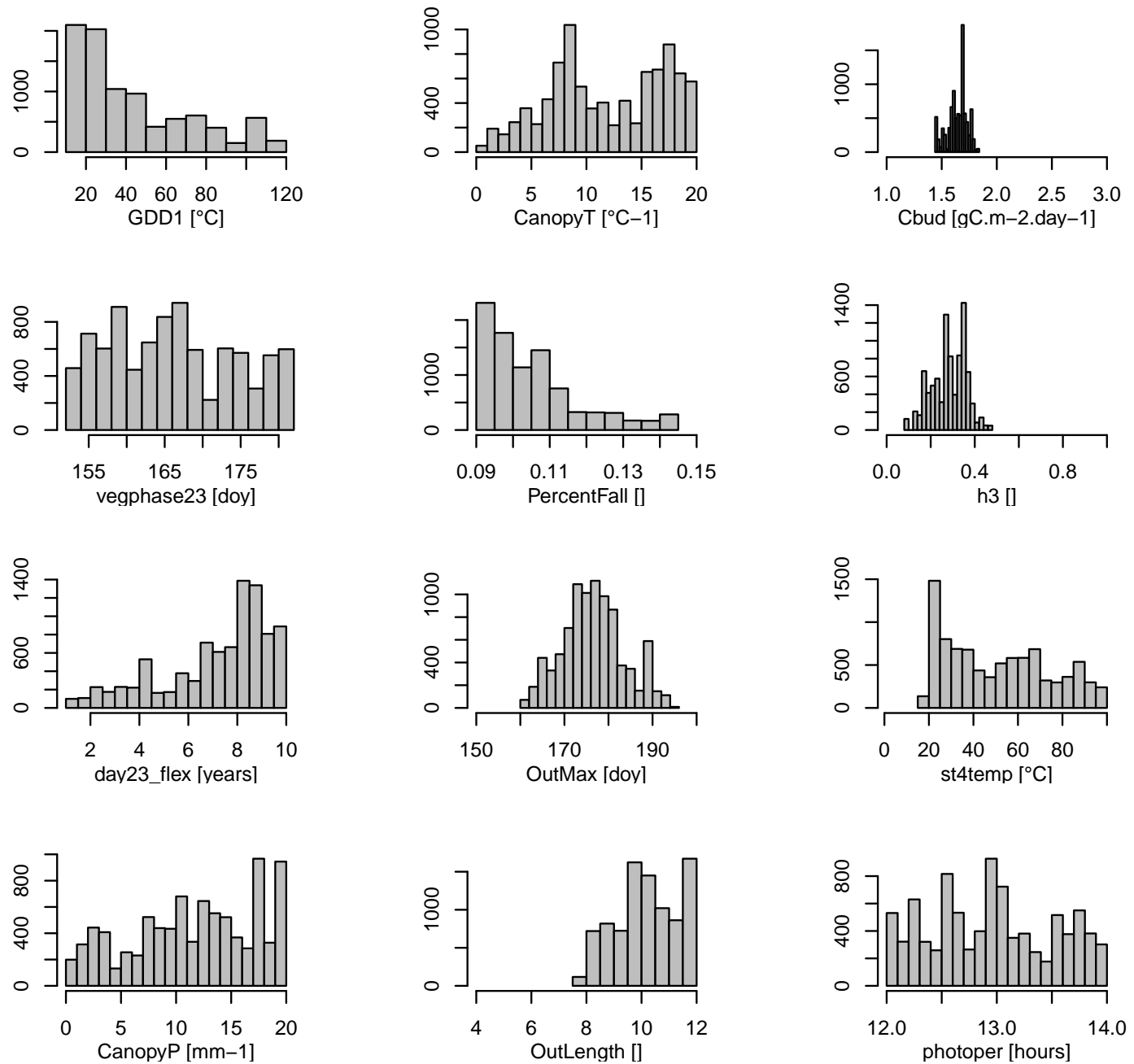

Figure S13. As in Fig. S1 at WHER site. 
Photosynthesis parameters for WHER
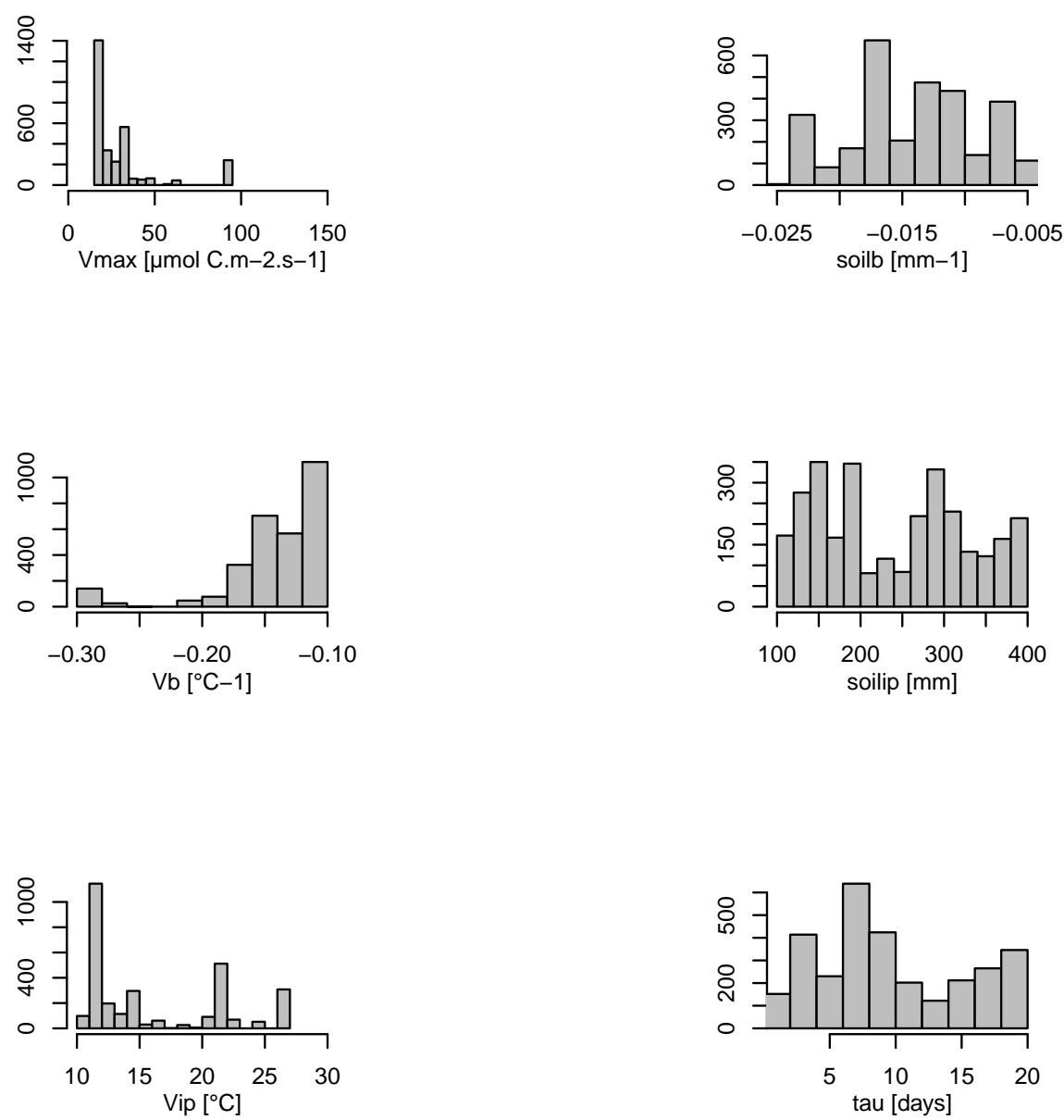

Figure S14. As in Fig. S2 at WHER site. 


\section{Carbon allocation parameters for WHH1}
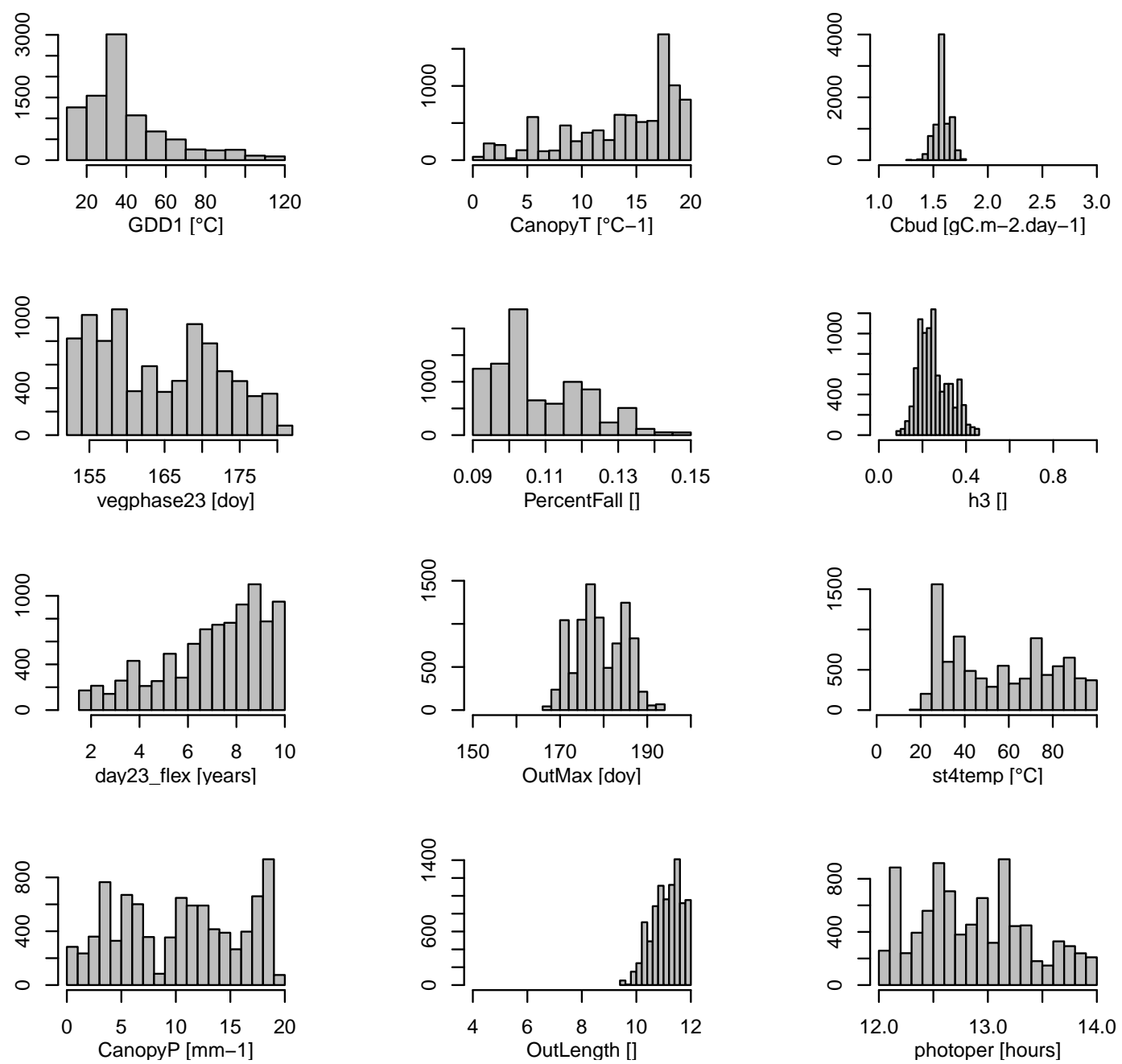

Figure S15. As in Fig. S1 at WHH1 site. 

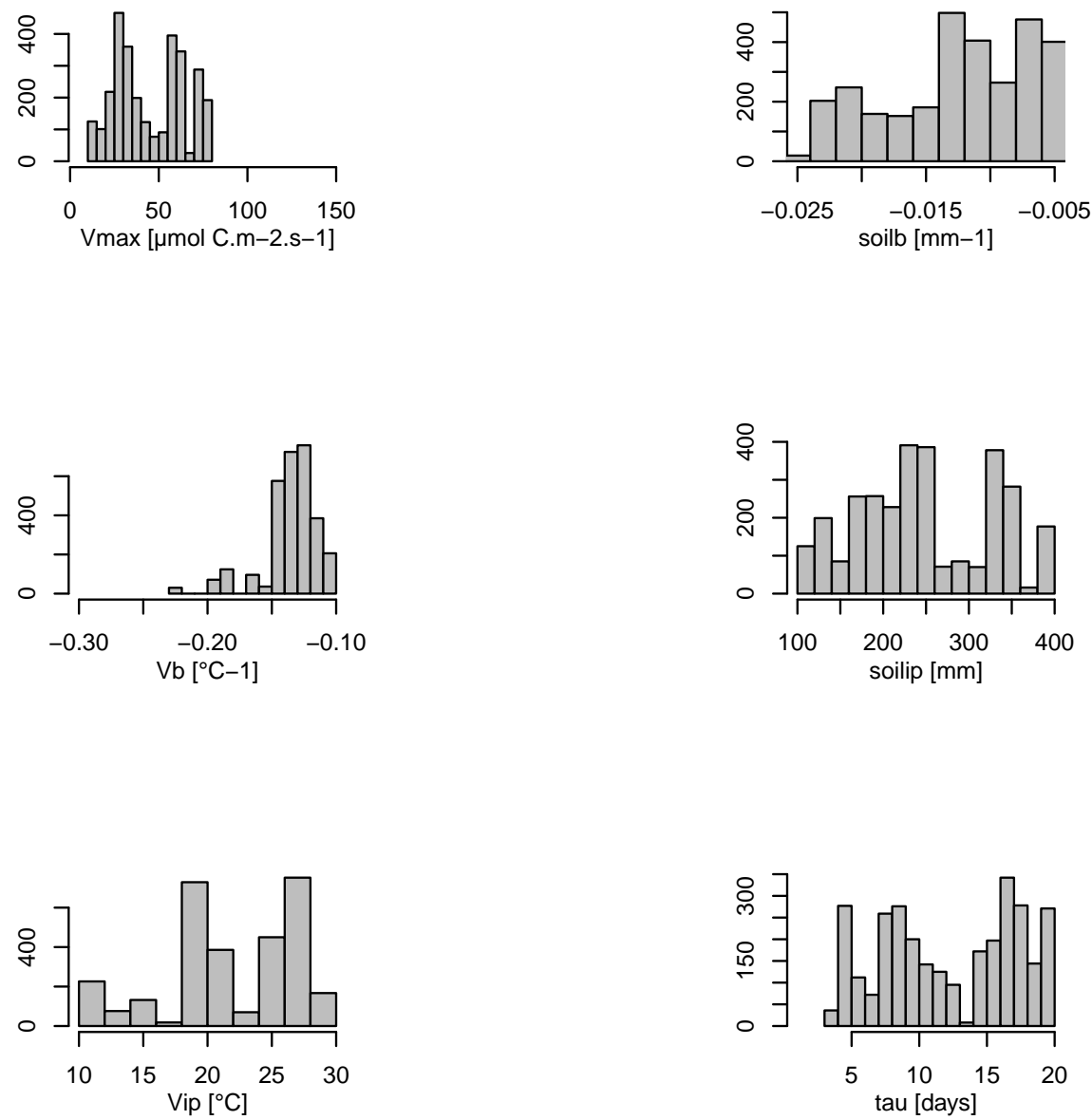

Figure S16. As in Fig. S2 at WHH1 site. 


\section{Carbon allocation parameters for WHM1}
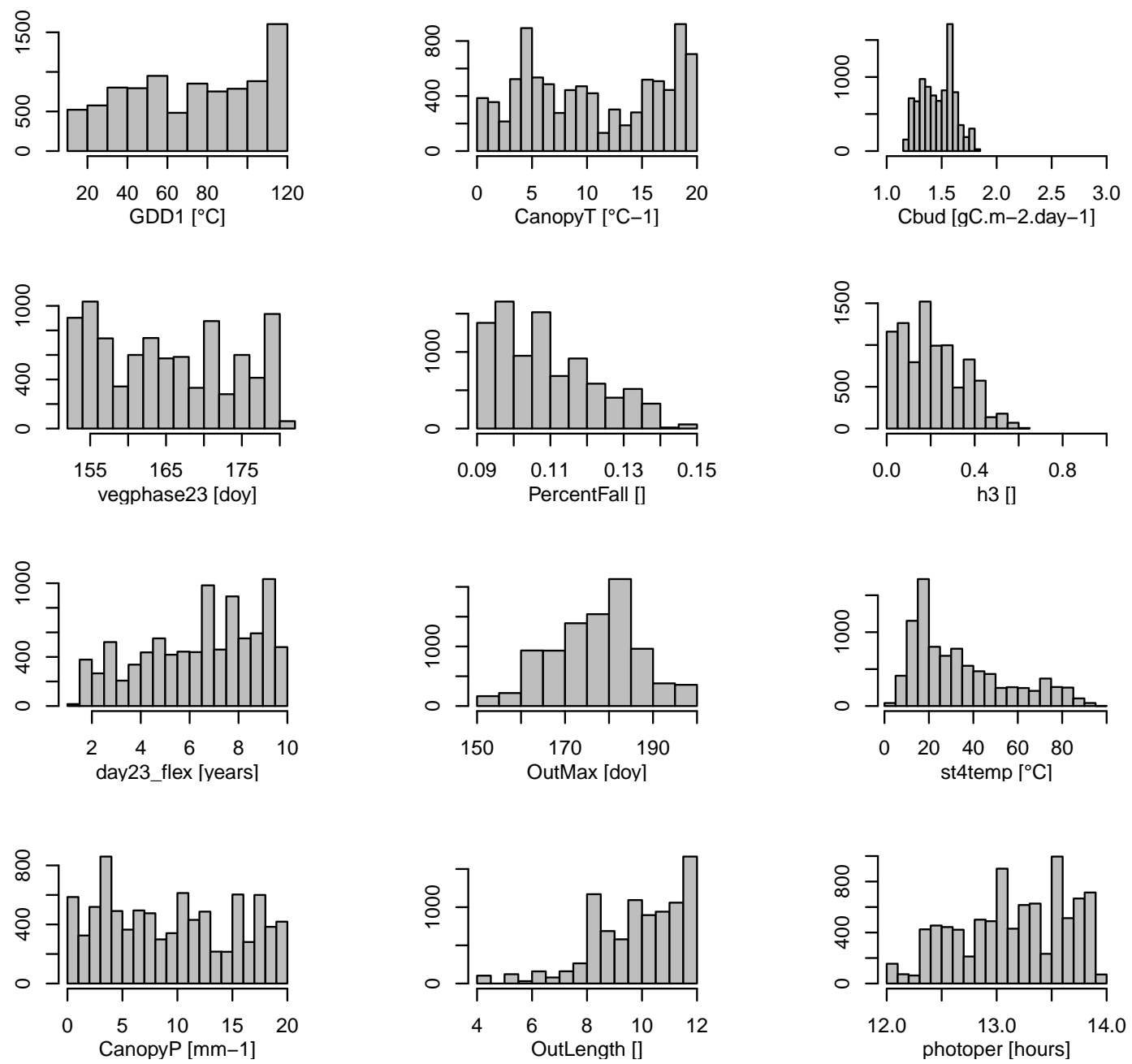

Figure S17. As in Fig. S1 at WHM1 site. 

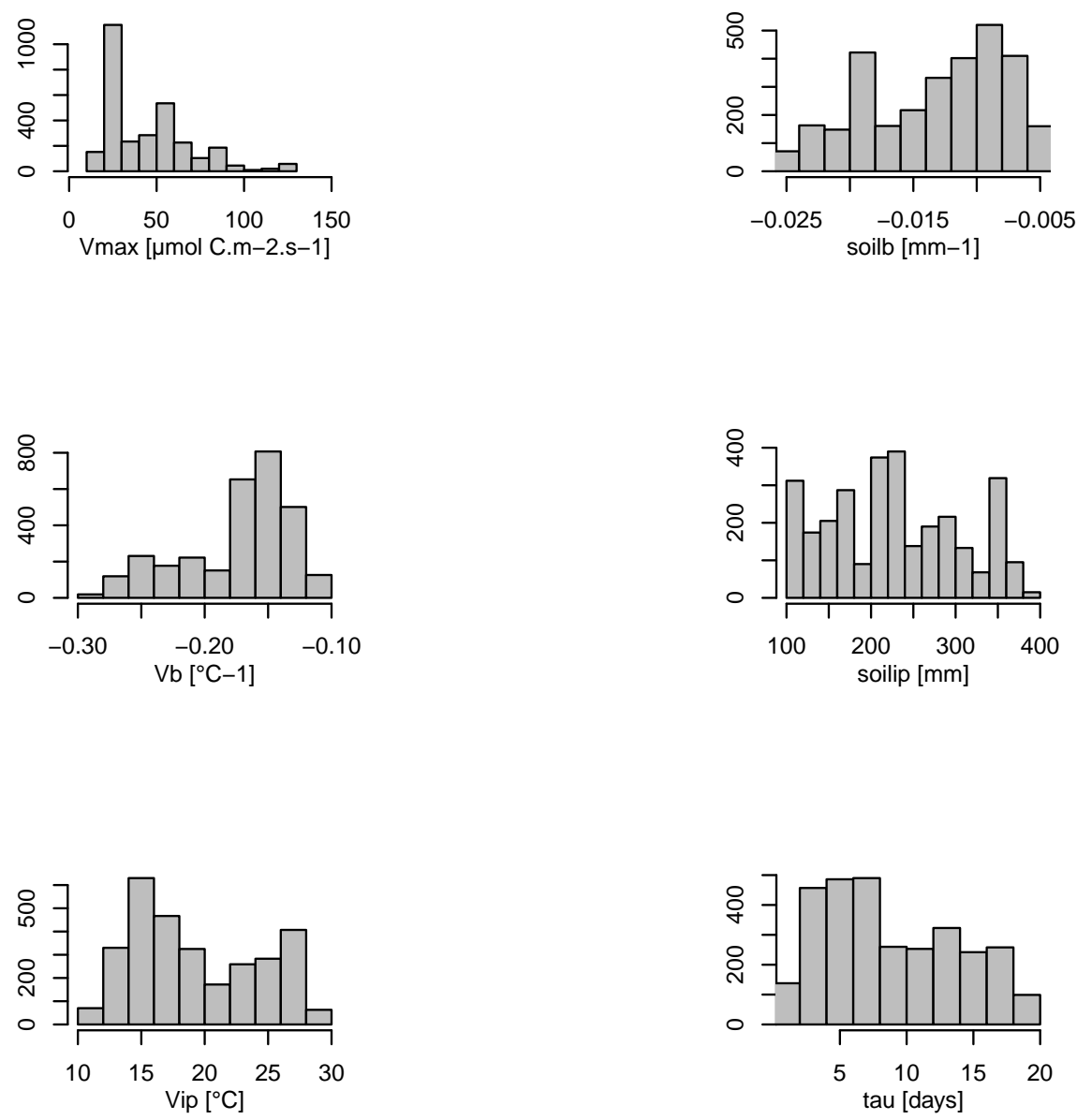

Figure S18. As in Fig. S2 at WHM1 site. 


\section{Carbon allocation parameters for WHM2}
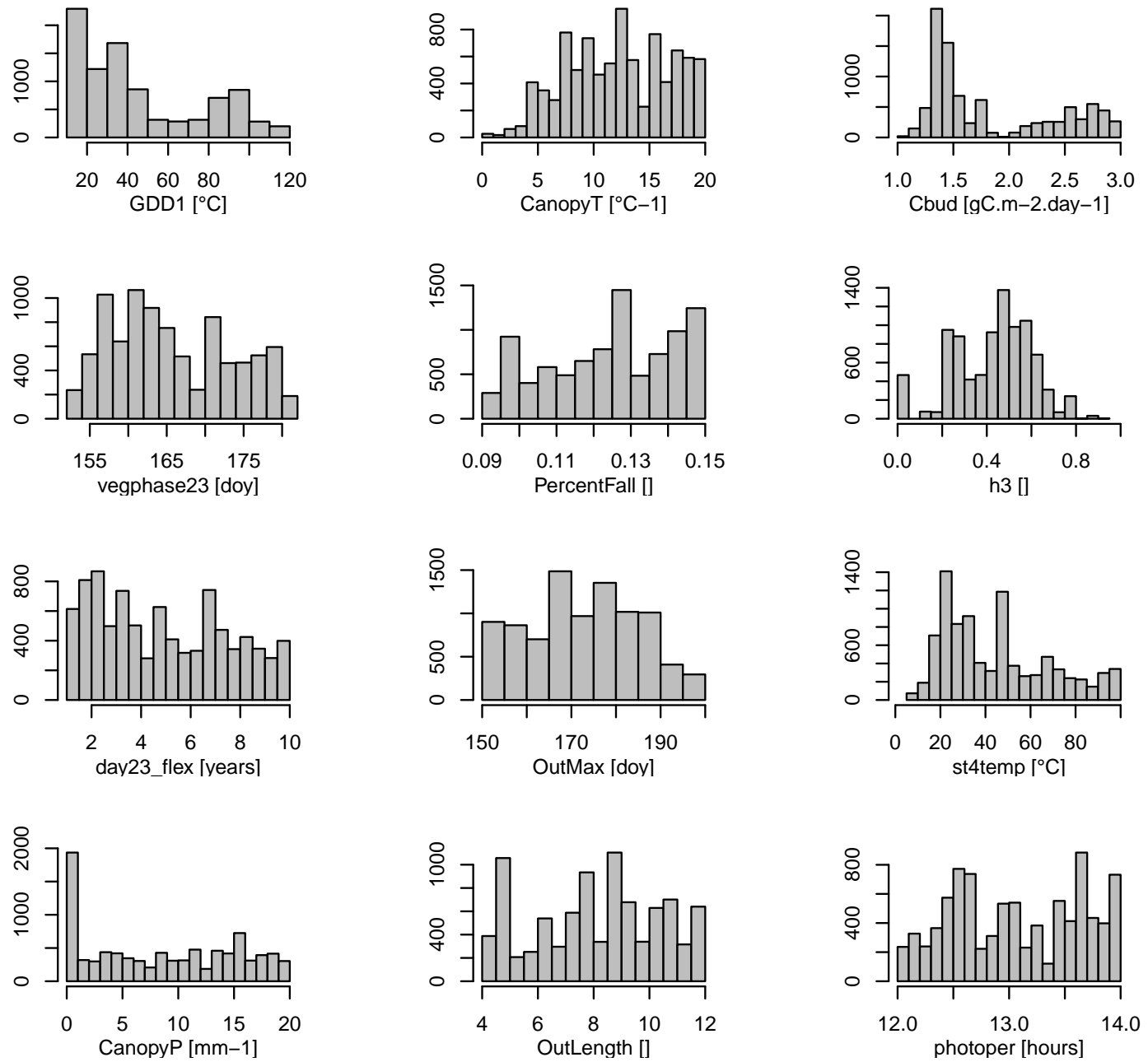

Figure S19. As in Fig. S1 at WHM2 site. 

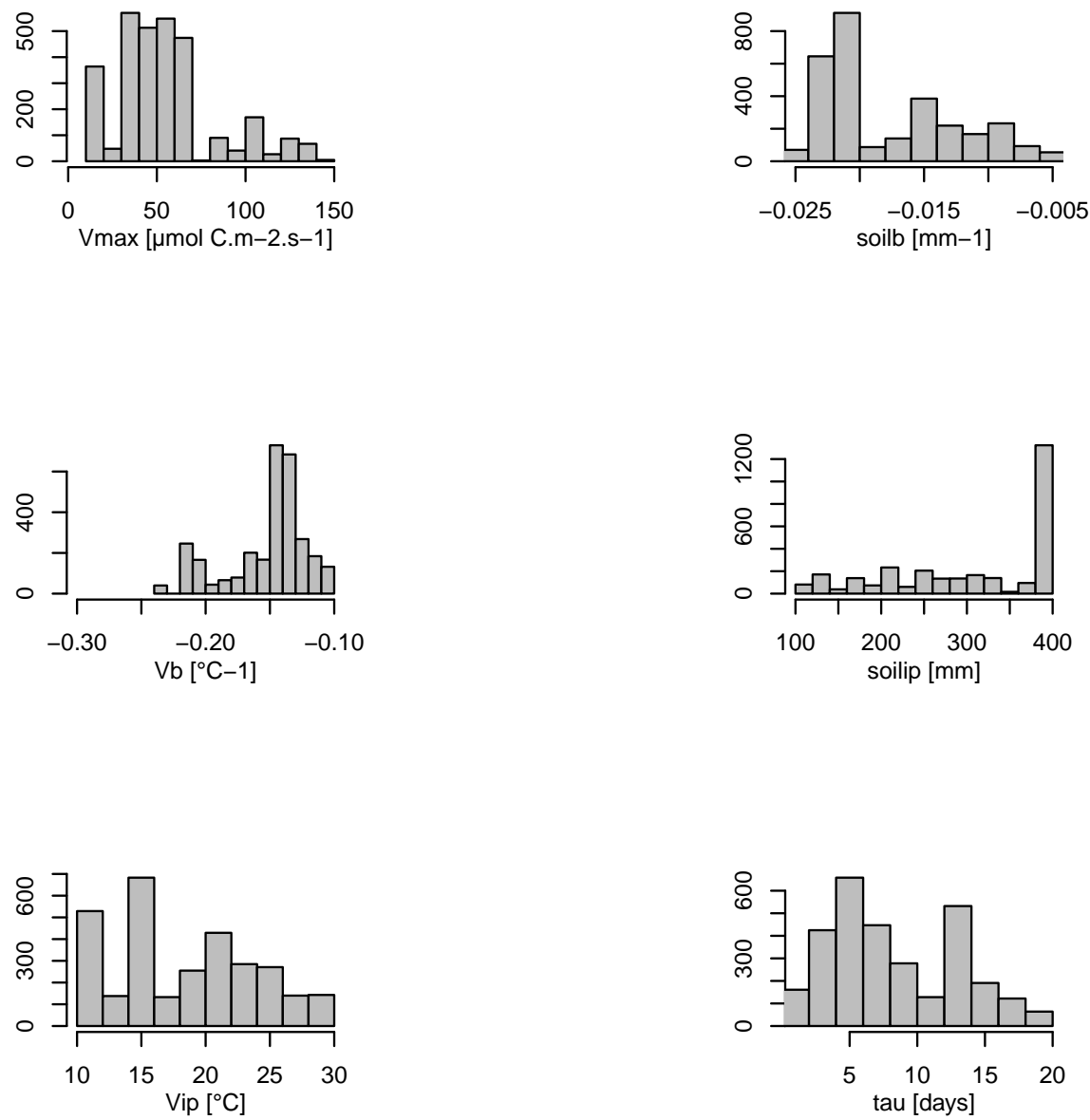

Figure S20. As in Fig. S2 at WHM2 site. 


\section{Carbon allocation parameters for WL32}
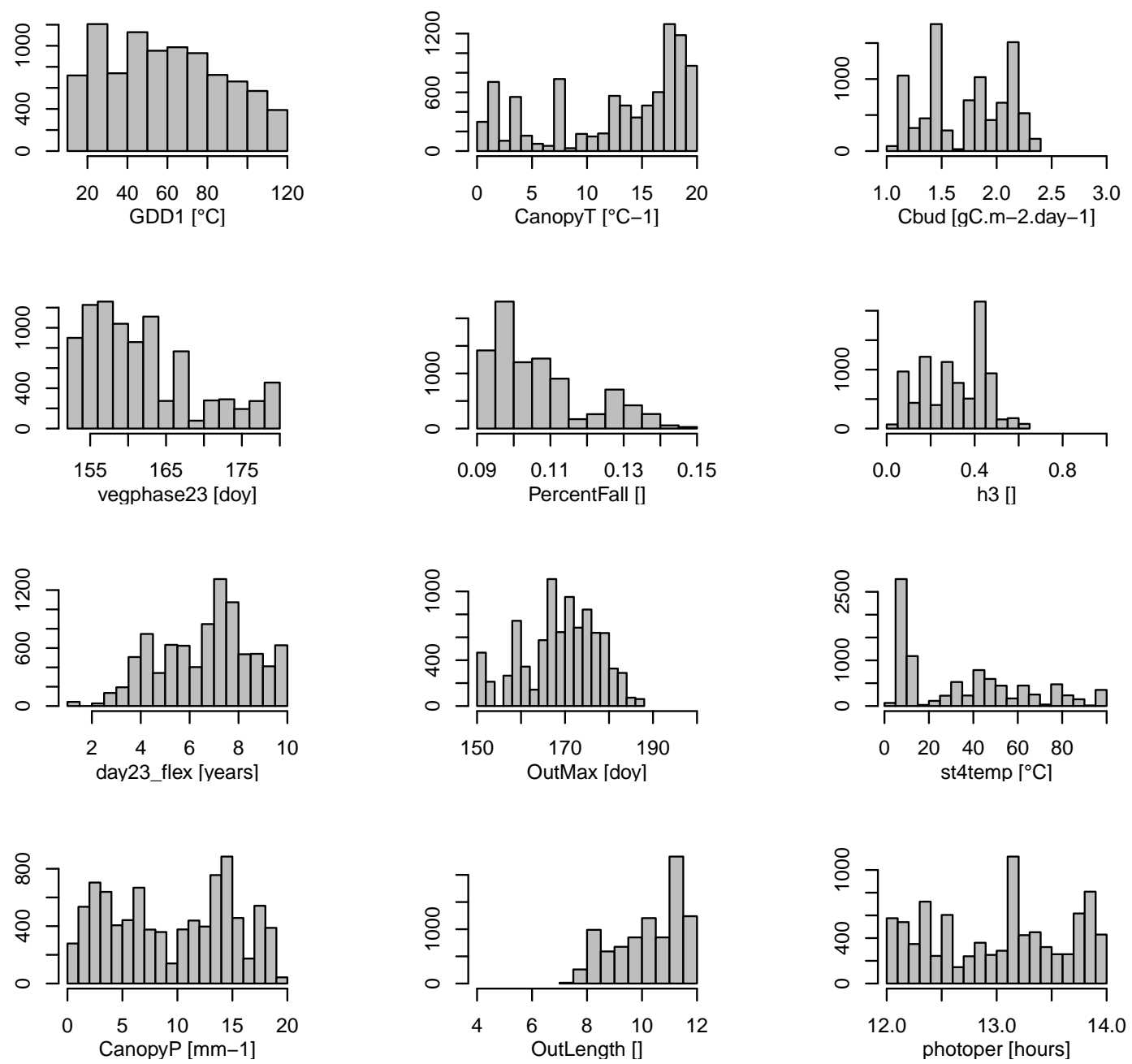

Figure S21. As in Fig. S1 at WL32 site. 
Photosynthesis parameters for WL32
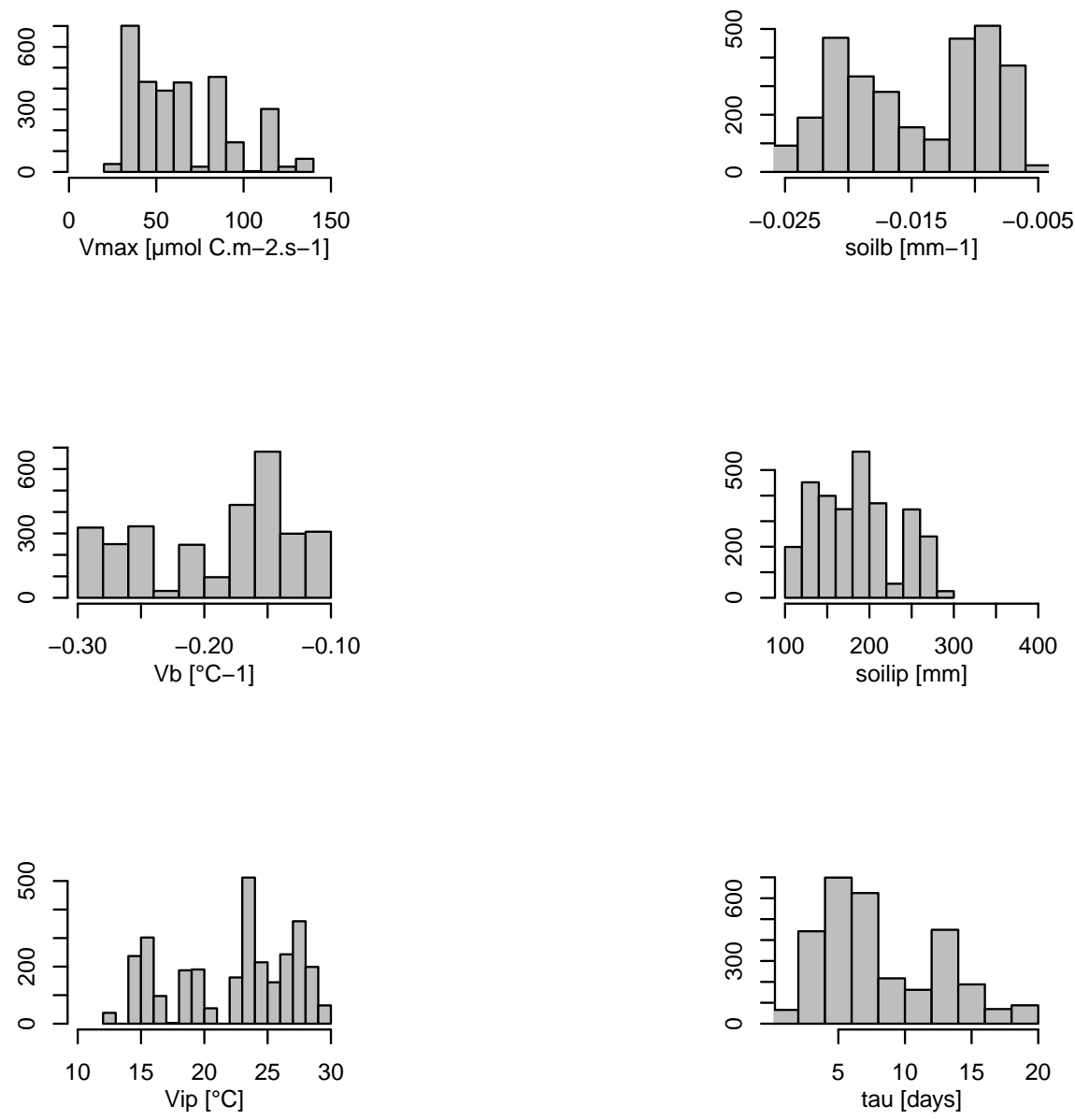

Figure S22. As in Fig. S2 at WL32 site. 


\section{Carbon allocation parameters for WL42}
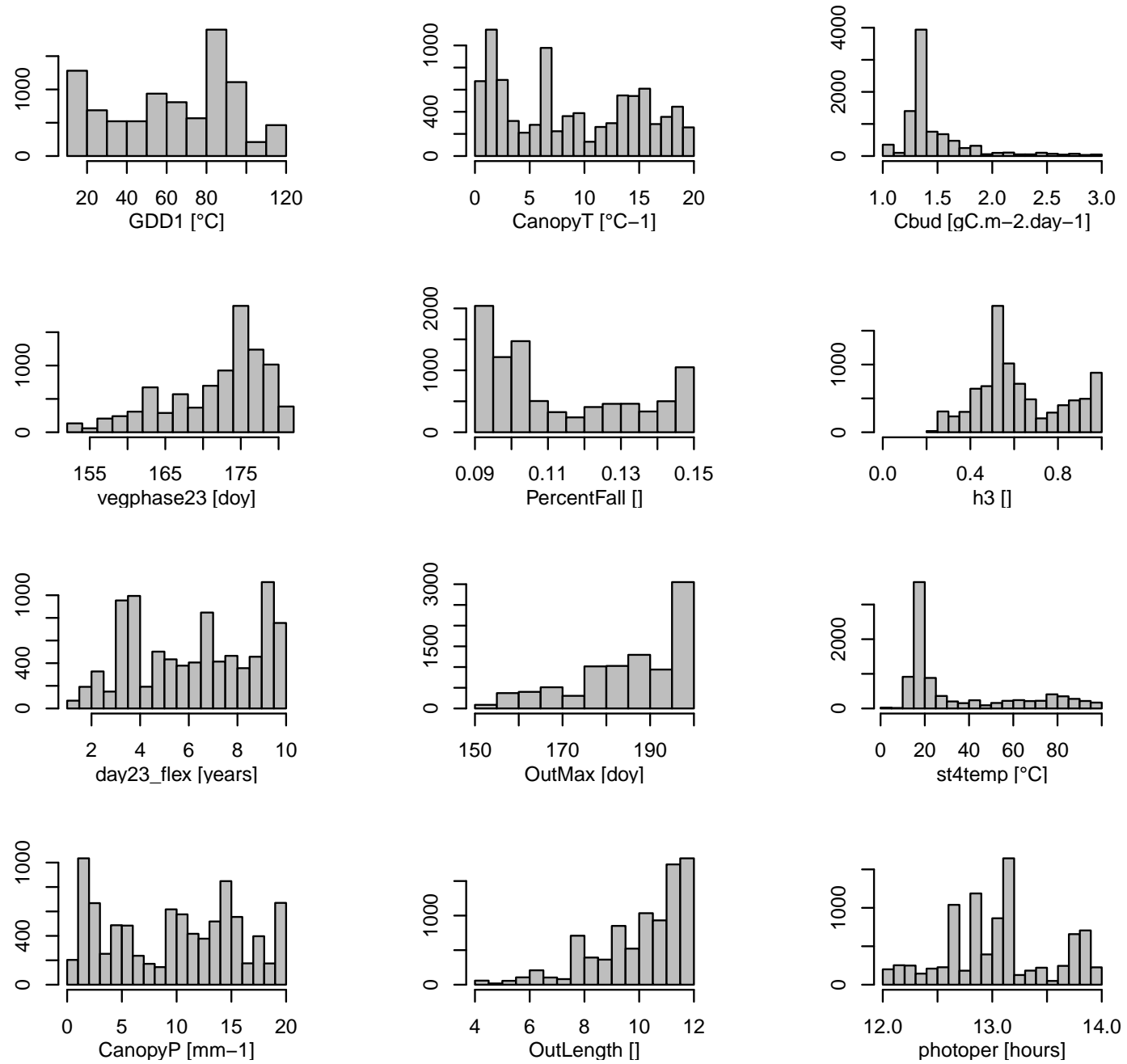

Figure S23. As in Fig. S1 at WL42 site. 
Photosynthesis parameters for WL42
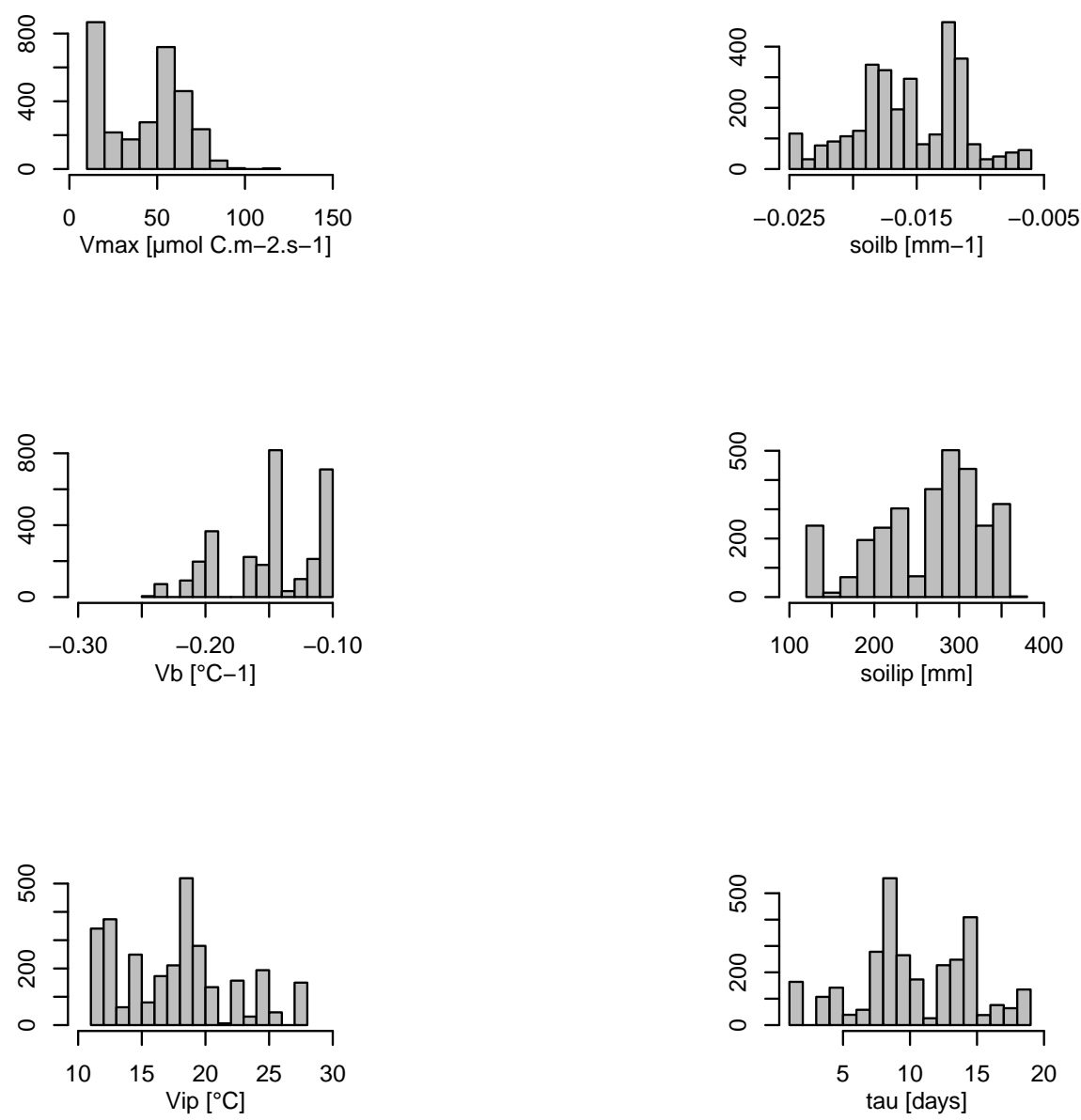

Figure S24. As in Fig. S2 at WL42 site. 
Carbon allocation parameters for WLECA
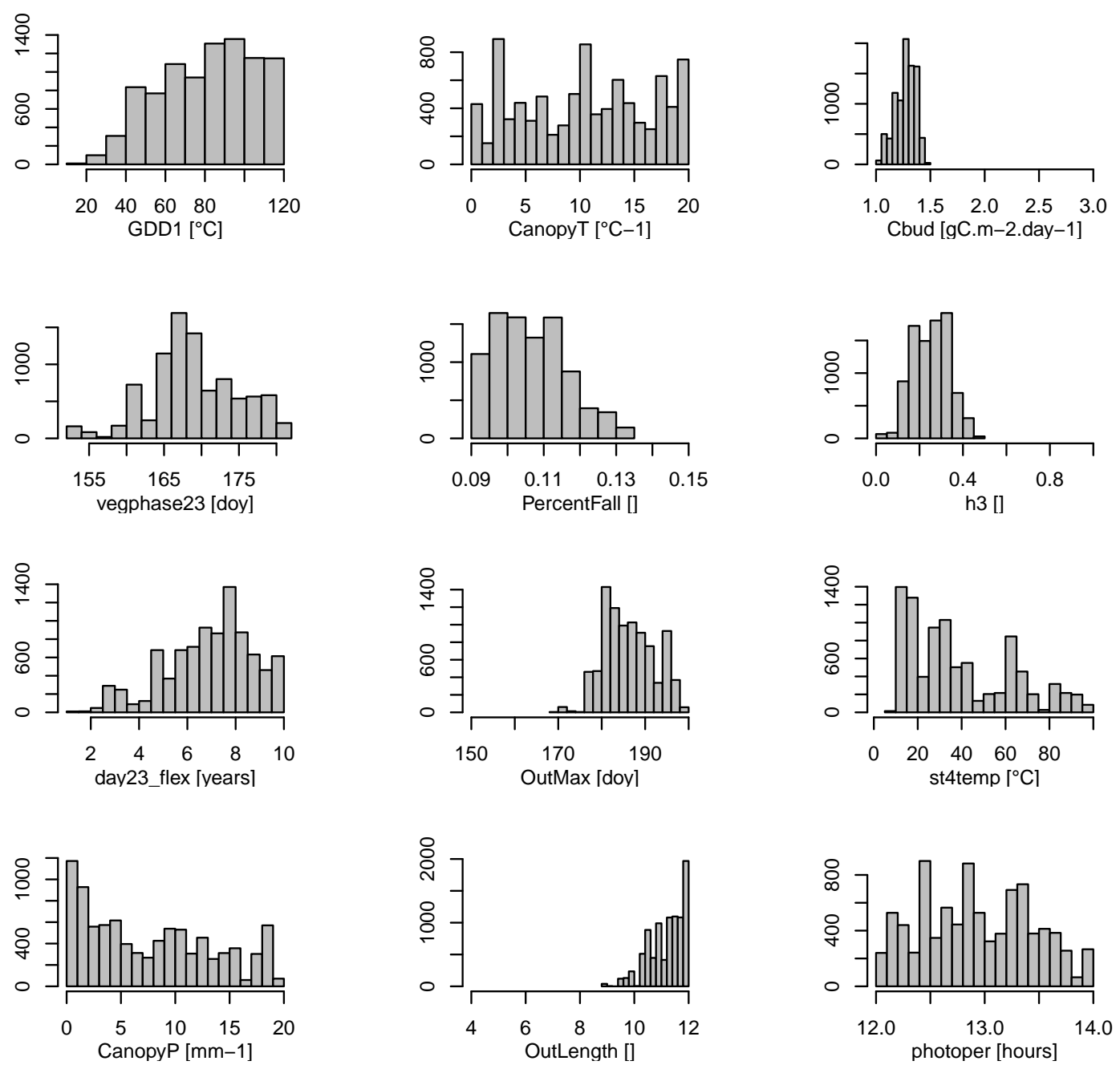

Figure S25. As in Fig. S1 at WLECA site. 
Photosynthesis parameters for WLECA
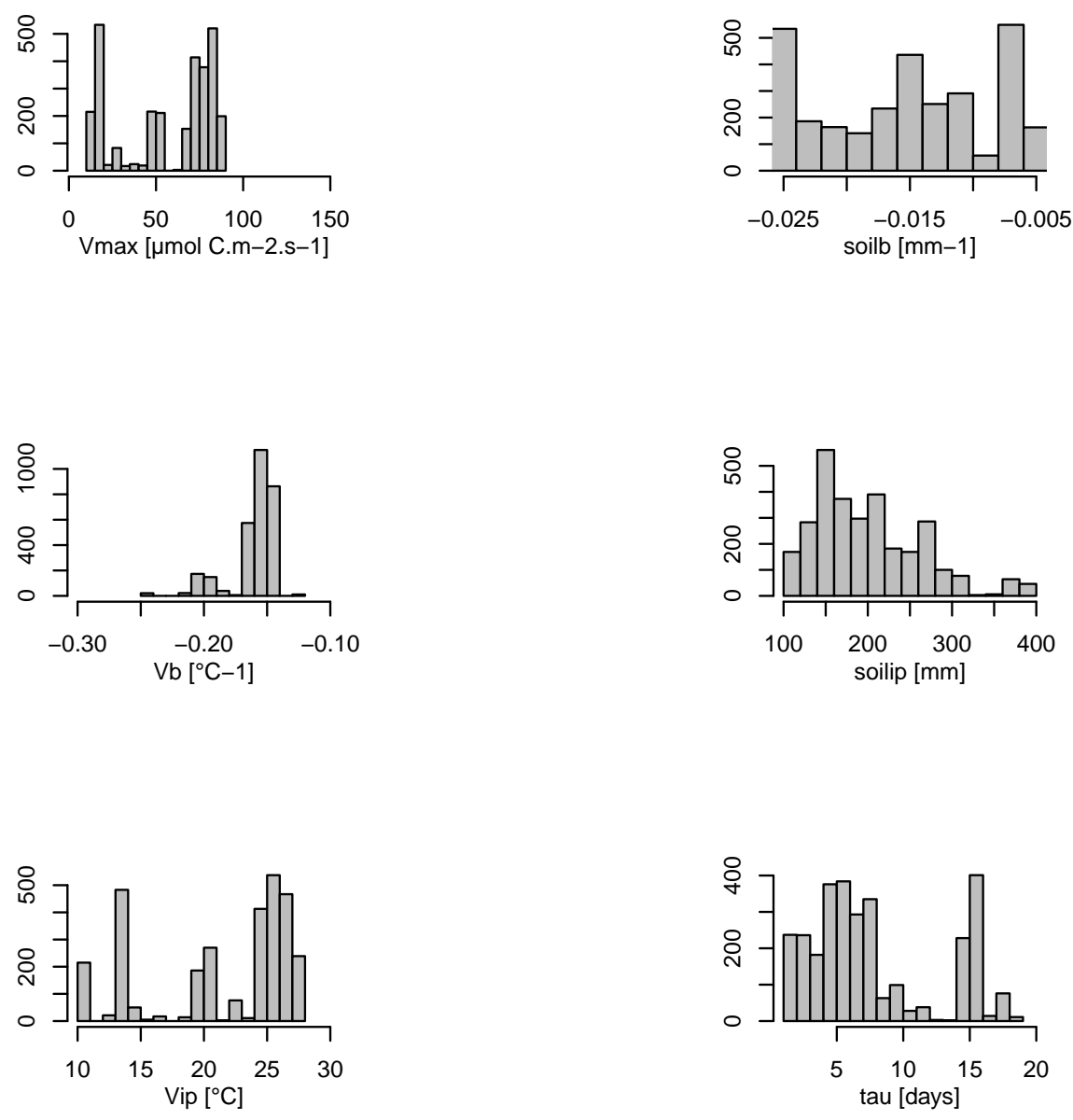

Figure S26. As in Fig. S2 at WLECA site. 
Carbon allocation parameters for WNFL1V
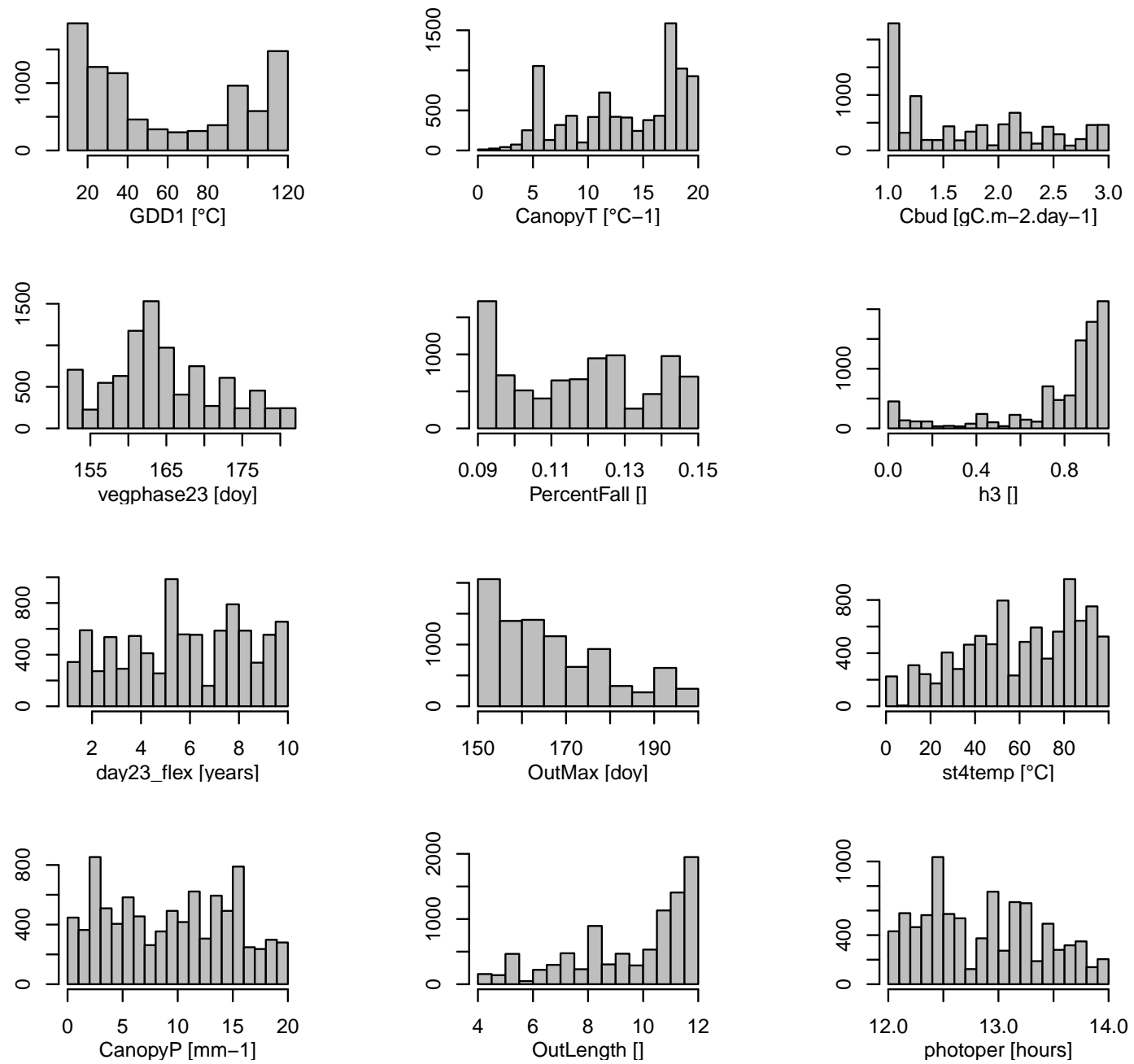

Figure S27. As in Fig. S1 at WNFL1V site. 

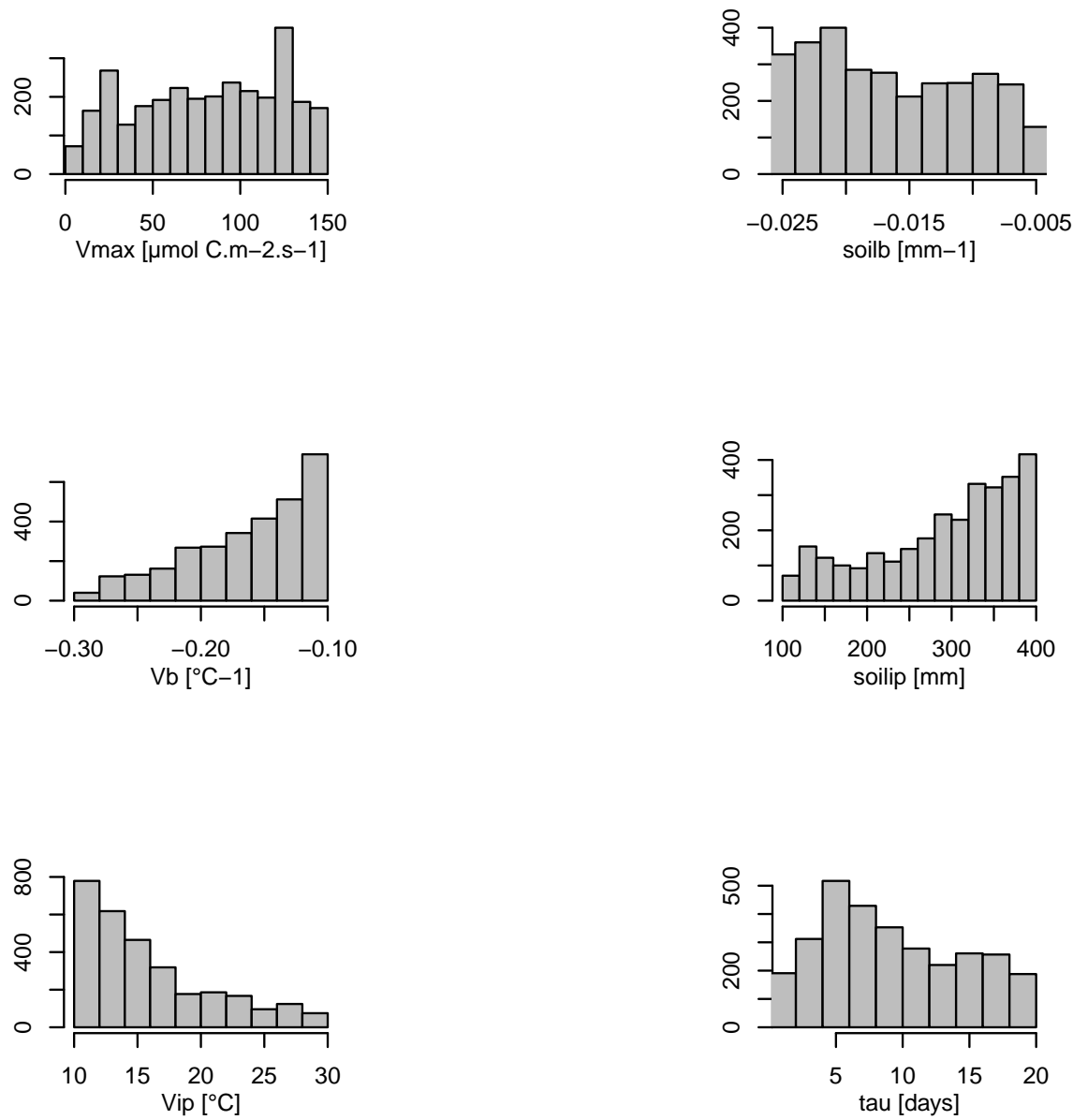

Figure S28. As in Fig. S2 at WNFL1V site. 

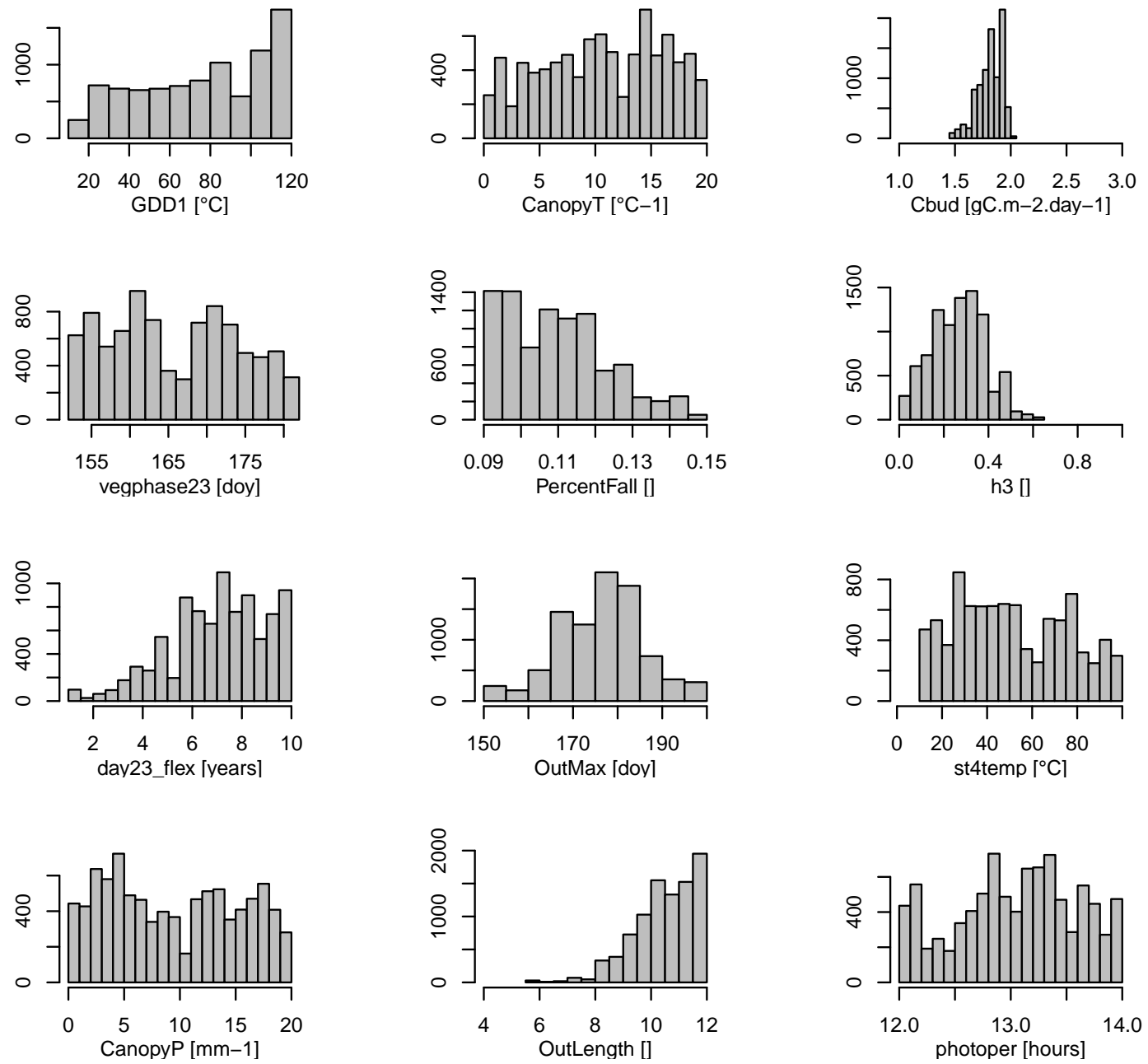

Figure S29. As in Fig. S1 at WNFLR1 site. 

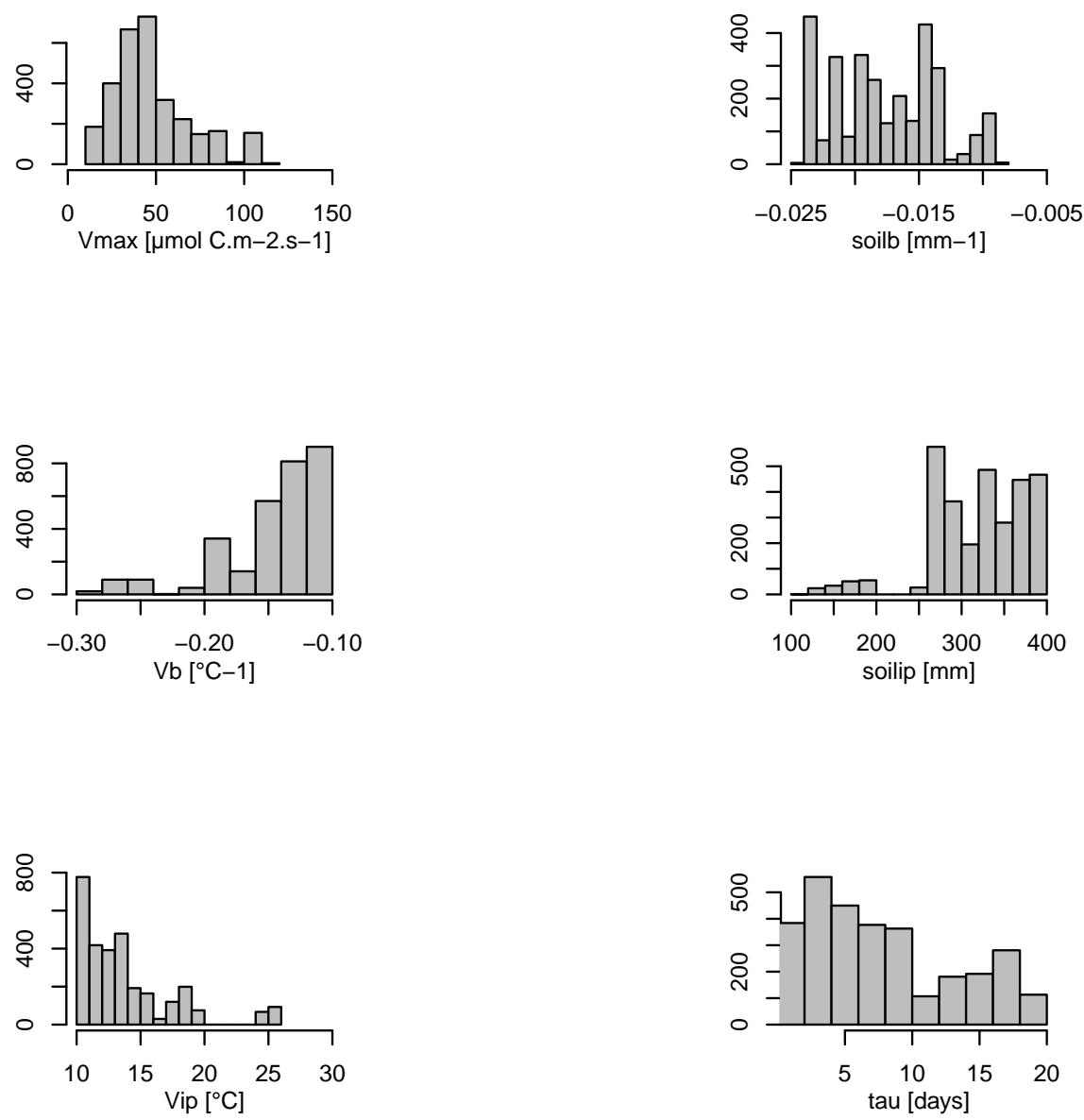

Figure S30. As in Fig. S2 at WNFLR1 site. 


\section{Carbon allocation parameters for WNIT}
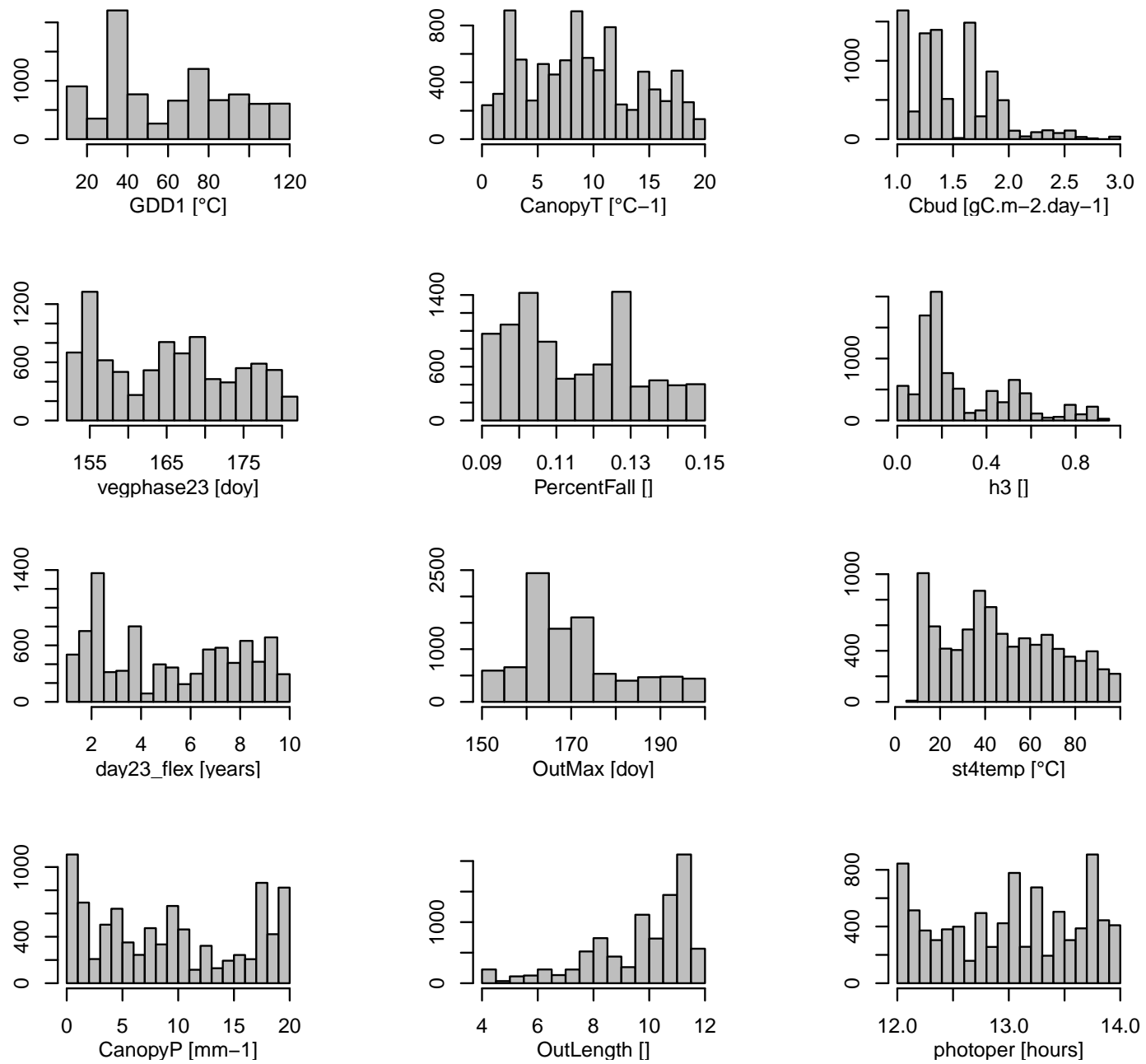

Figure S31. As in Fig. S1 at WNIT site. 

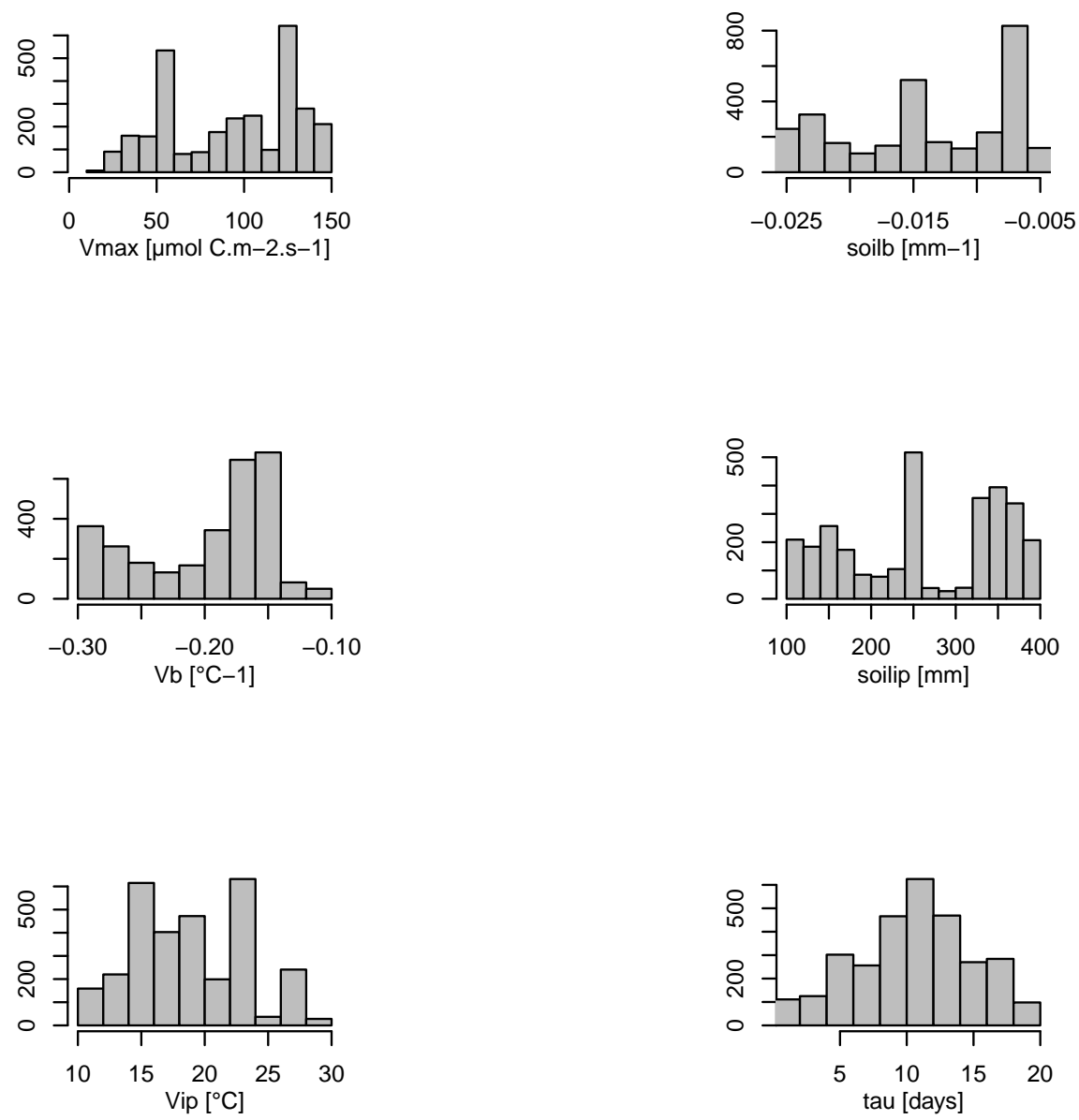

Figure S32. As in Fig. S2 at WNIT site. 
Carbon allocation parameters for WPOOL
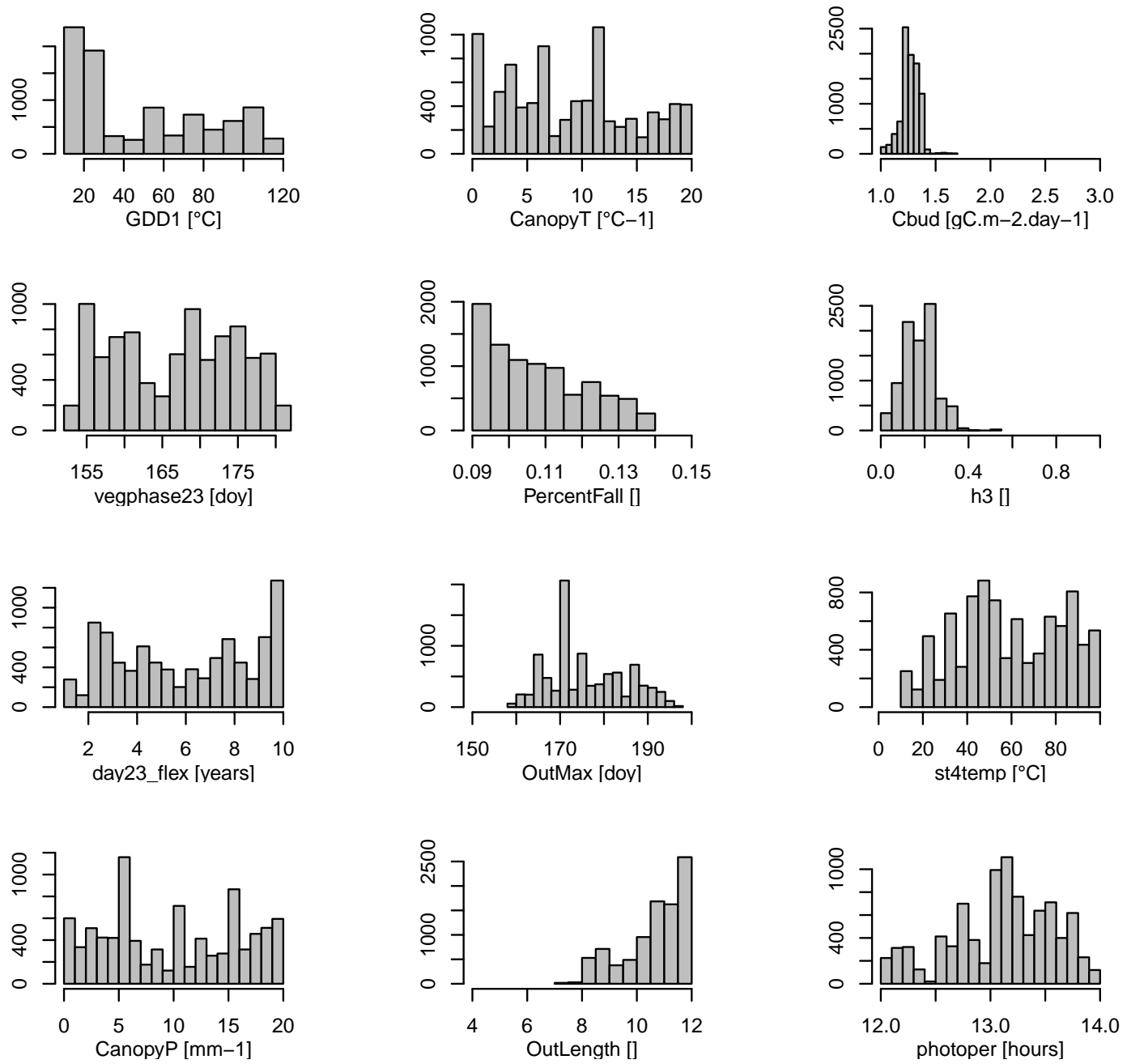

Figure S33. As in Fig. S1 at WPOOL site. 
Photosynthesis parameters for WPOOL
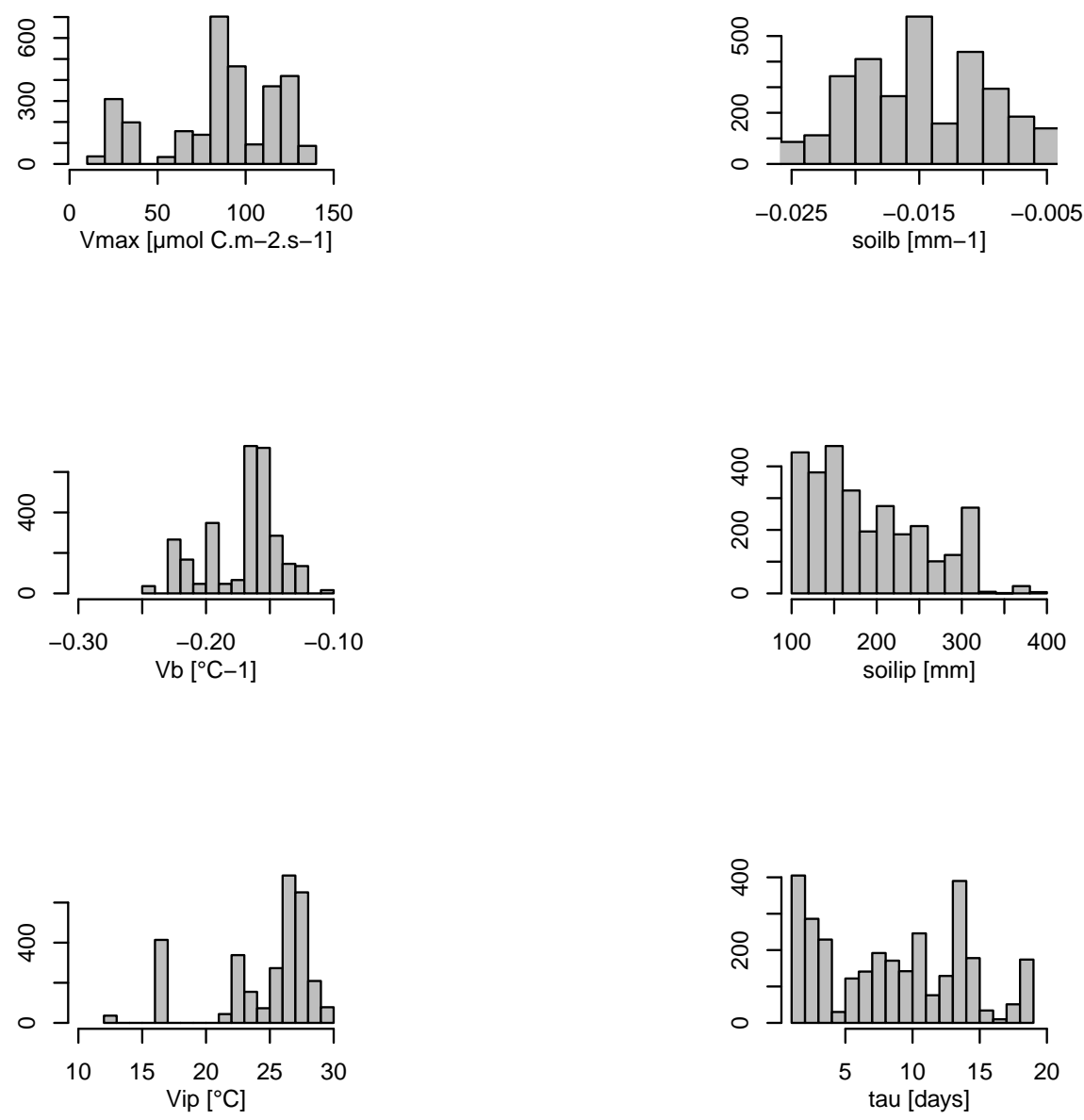

Figure S34. As in Fig. S2 at WPOOL site. 


\section{Carbon allocation parameters for WROZM}
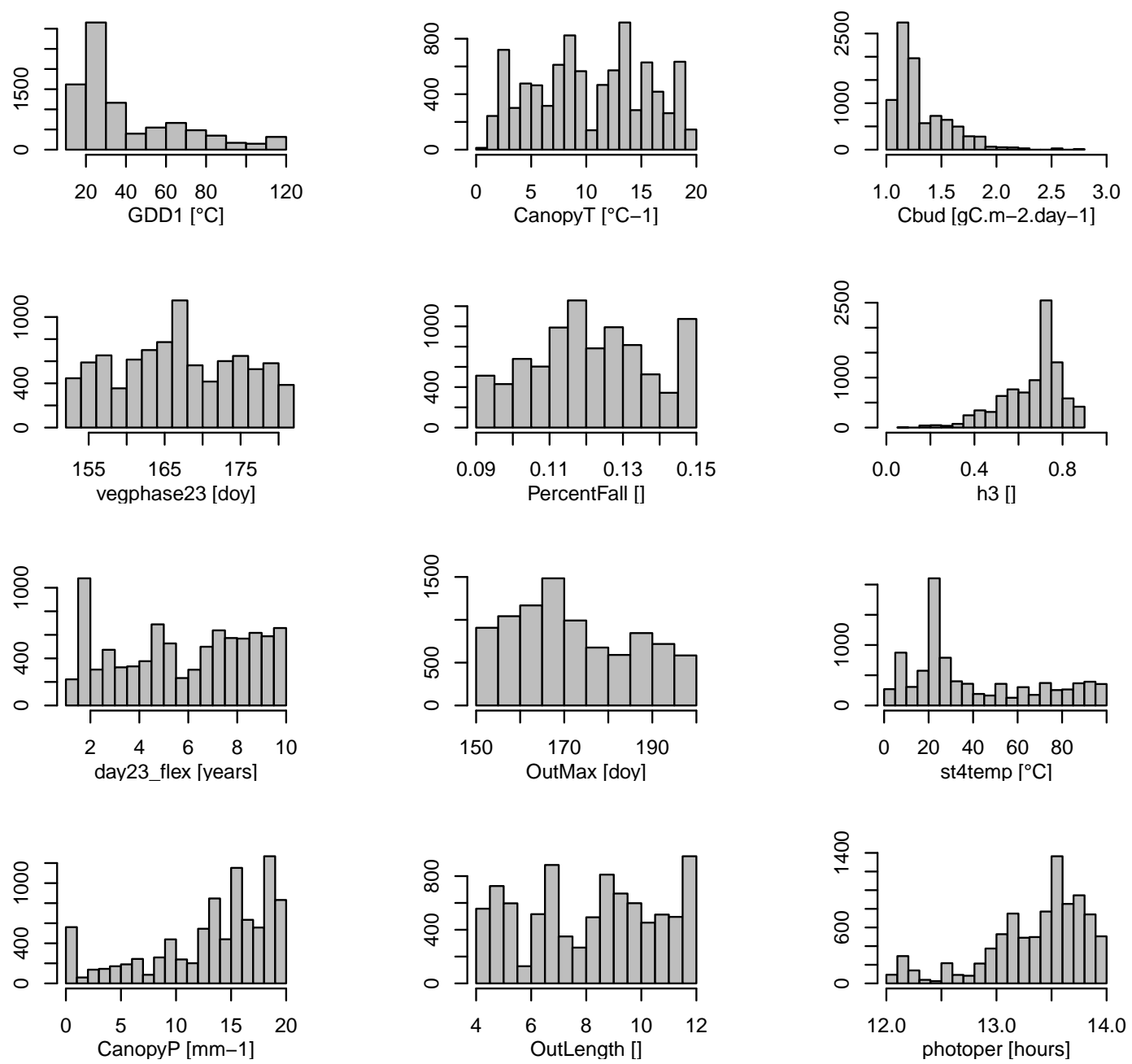

Figure S35. As in Fig. S1 at WROZM site. 

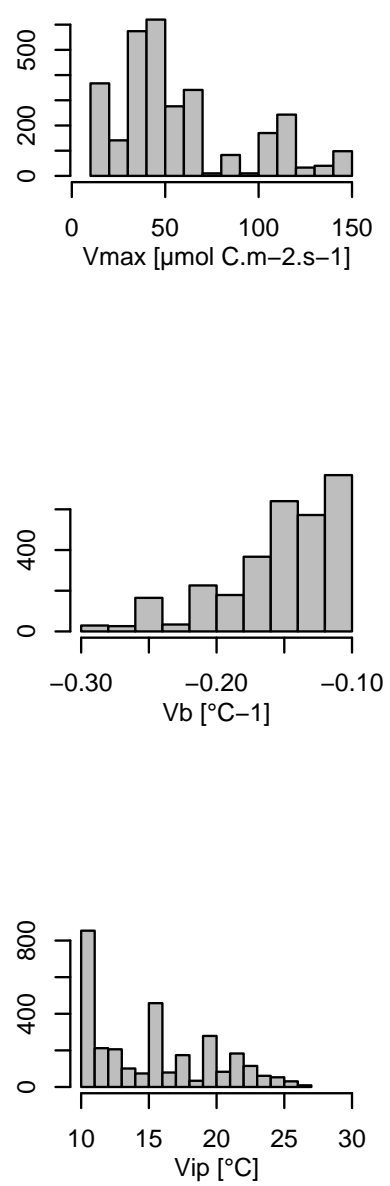
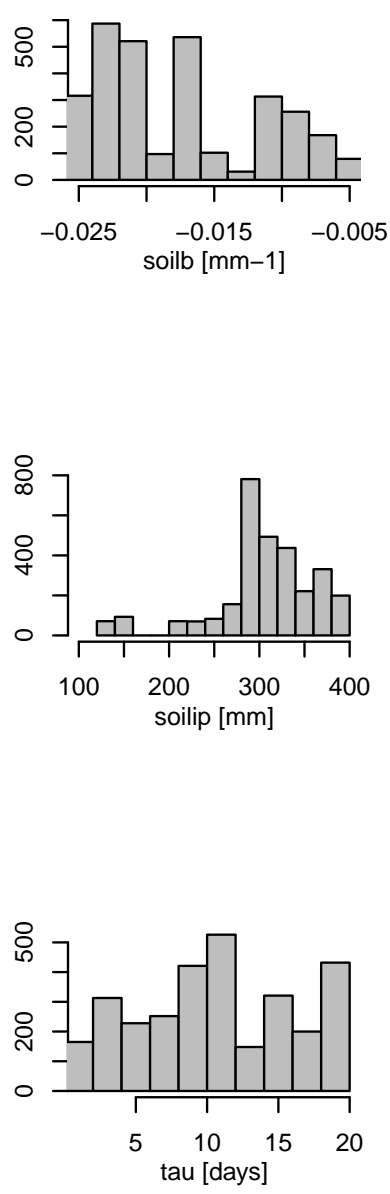

Figure S36. As in Fig. S2 at WROZM site. 

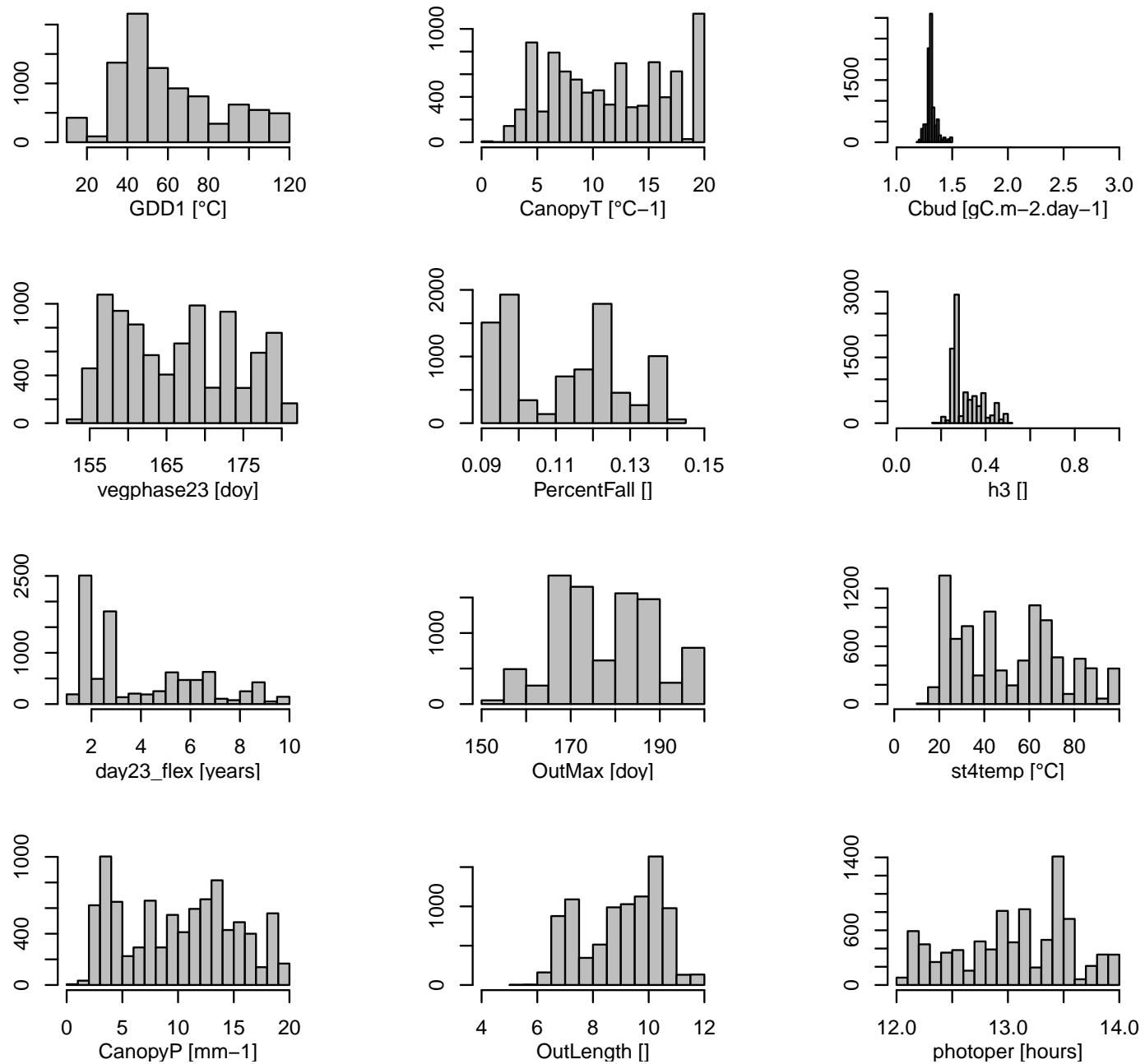

Figure S37. As in Fig. S1 at WROZX site. 

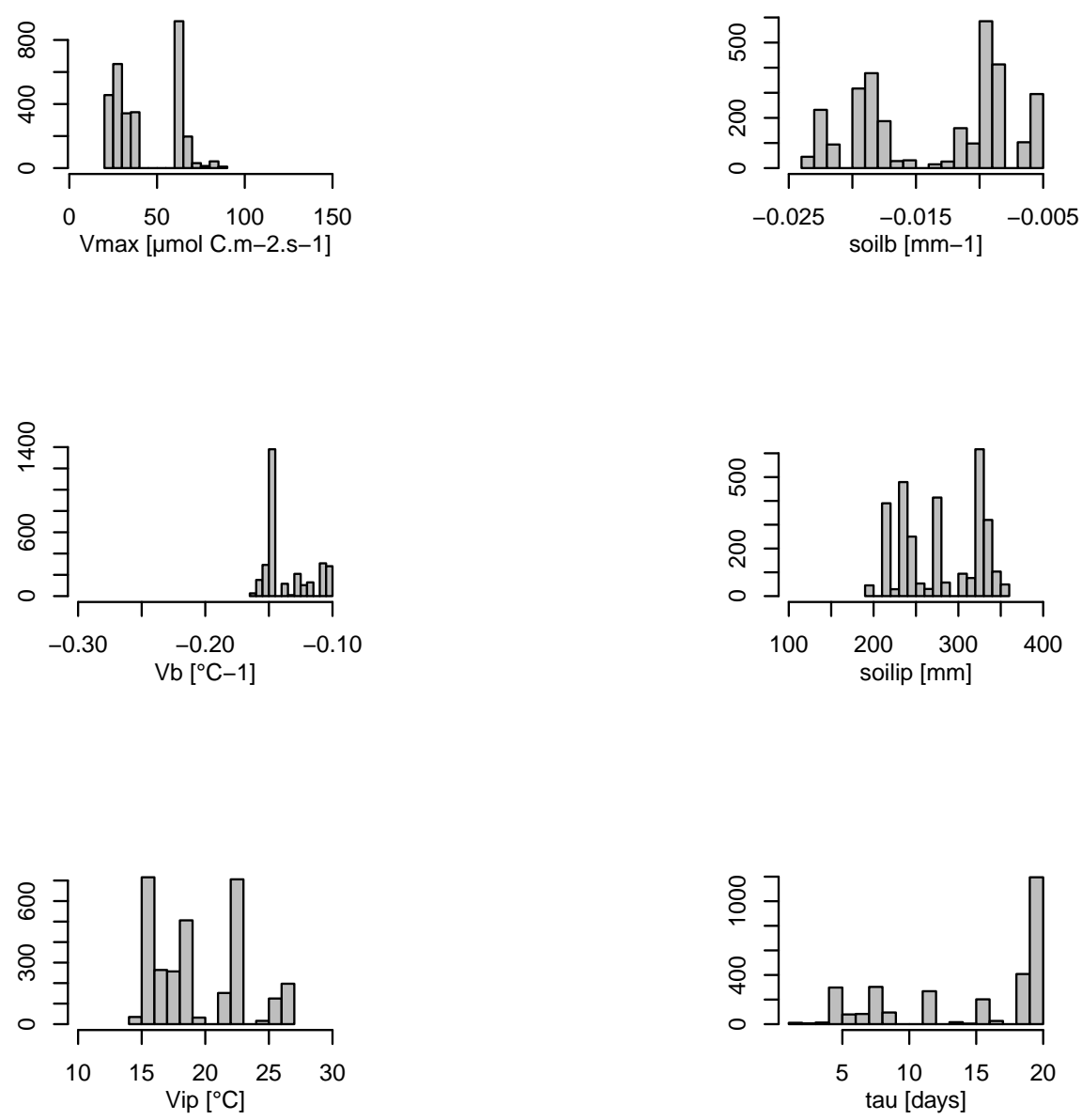

Figure S38. As in Fig. S2 at WROZX site. 


\section{Carbon allocation parameters for WRT485}
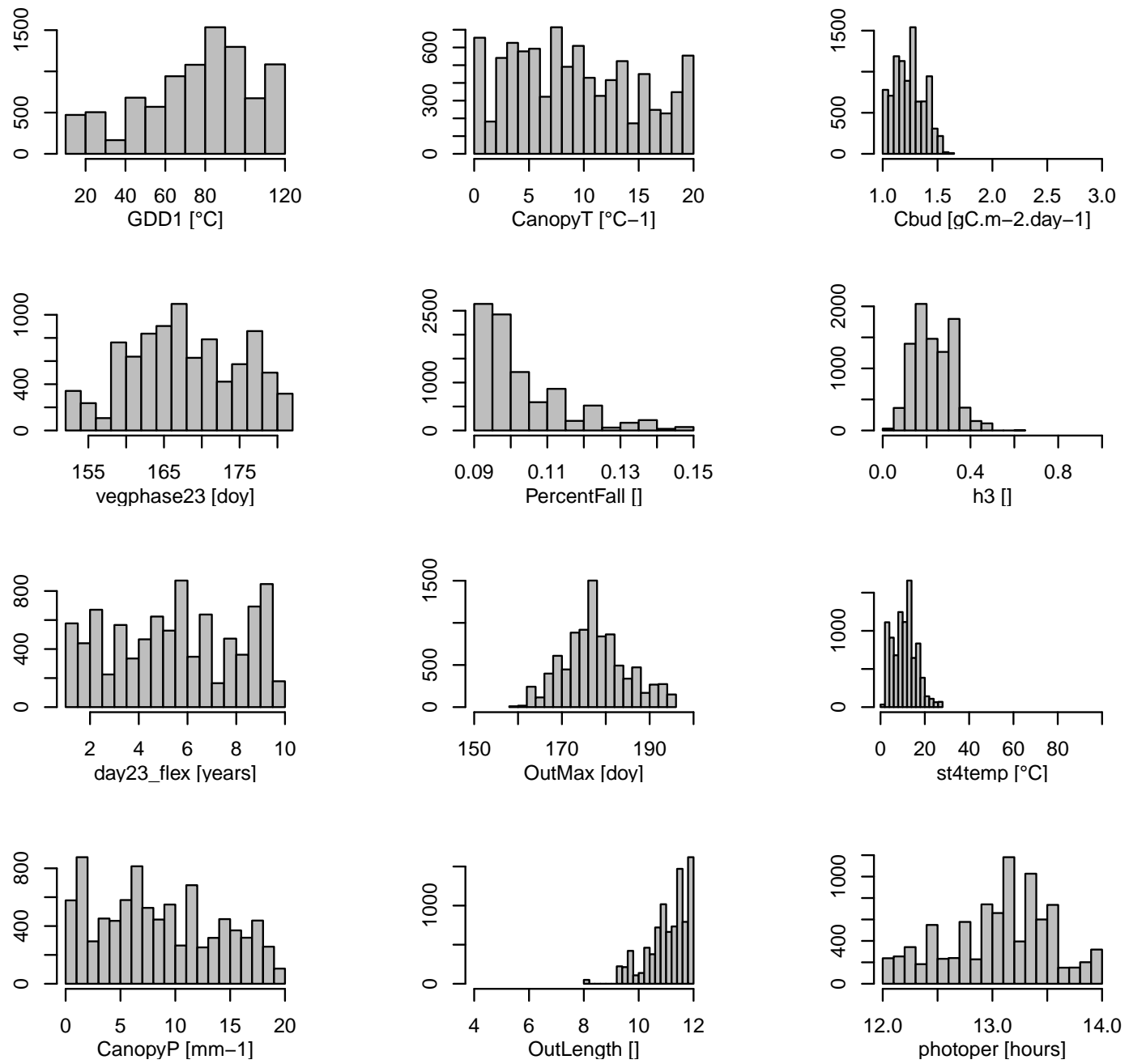

Figure S39. As in Fig. S1 at WRT485 site. 
Photosynthesis parameters for WRT485
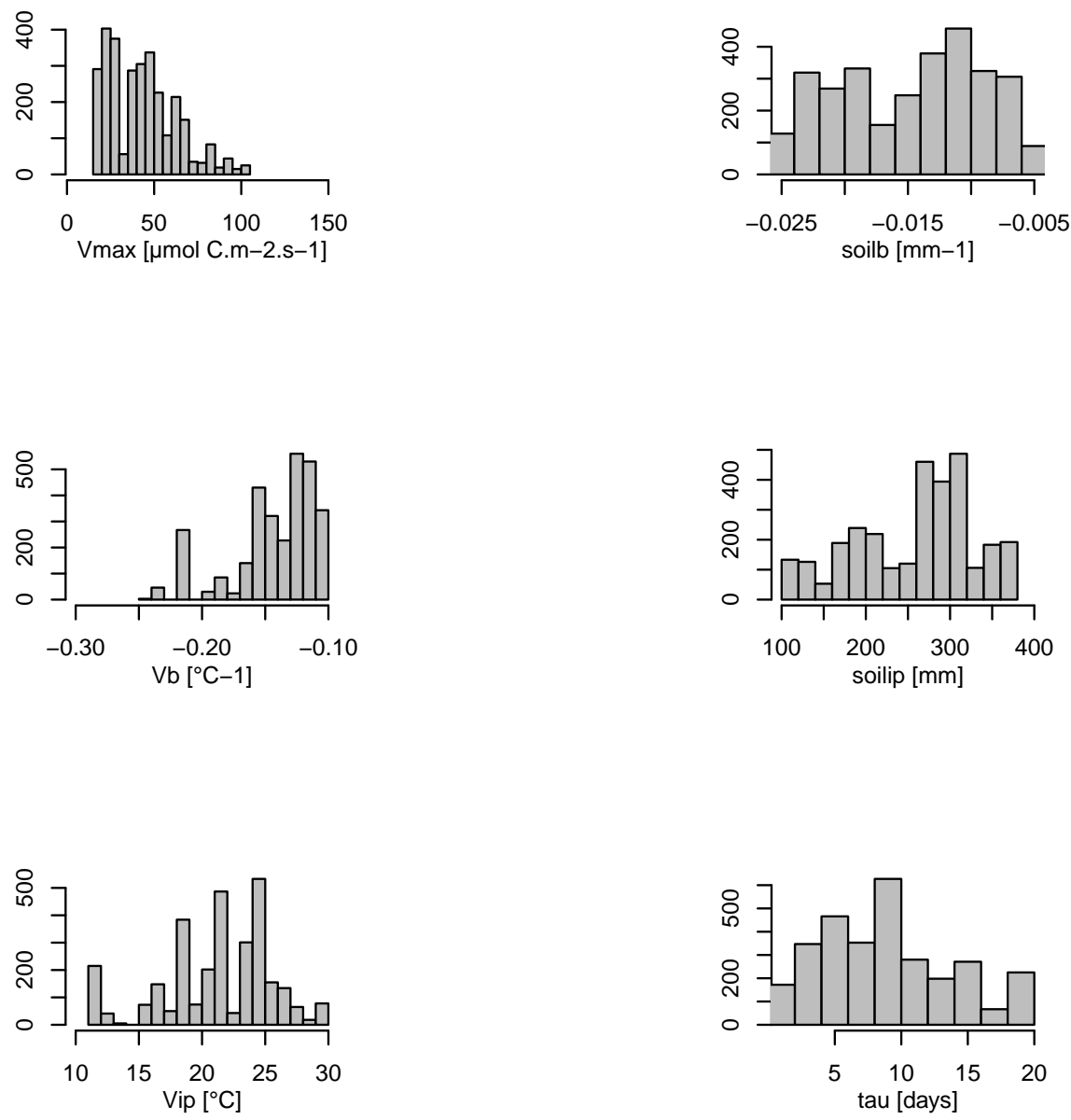

Figure S40. As in Fig. S2 at WRT485 site. 


\section{Carbon allocation parameters for WTHH}
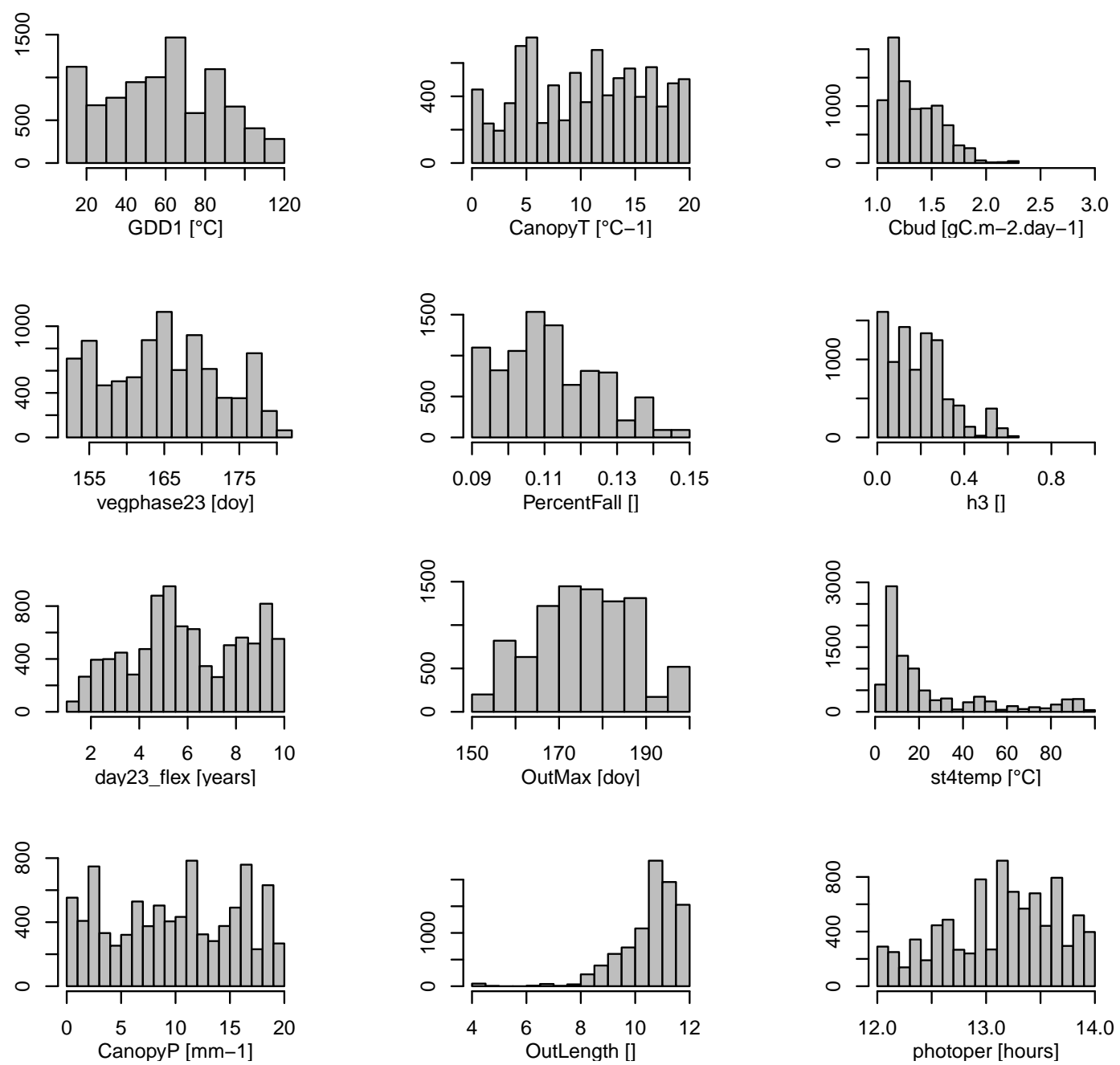

Figure S41. As in Fig. S1 at WTHH site. 

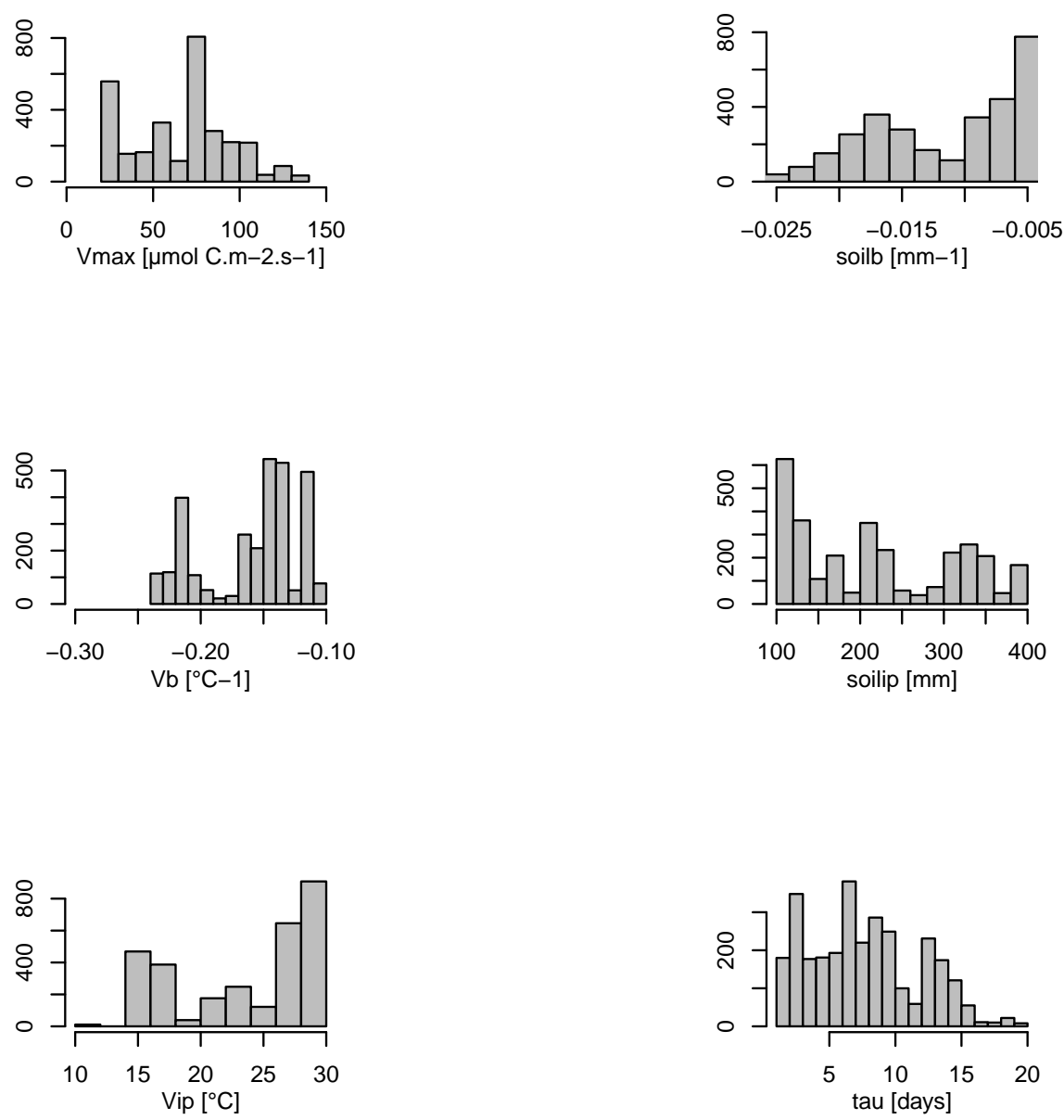

Figure S42. As in Fig. S2 at WTHH site. 


\section{Carbon allocation parameters for WROZ}
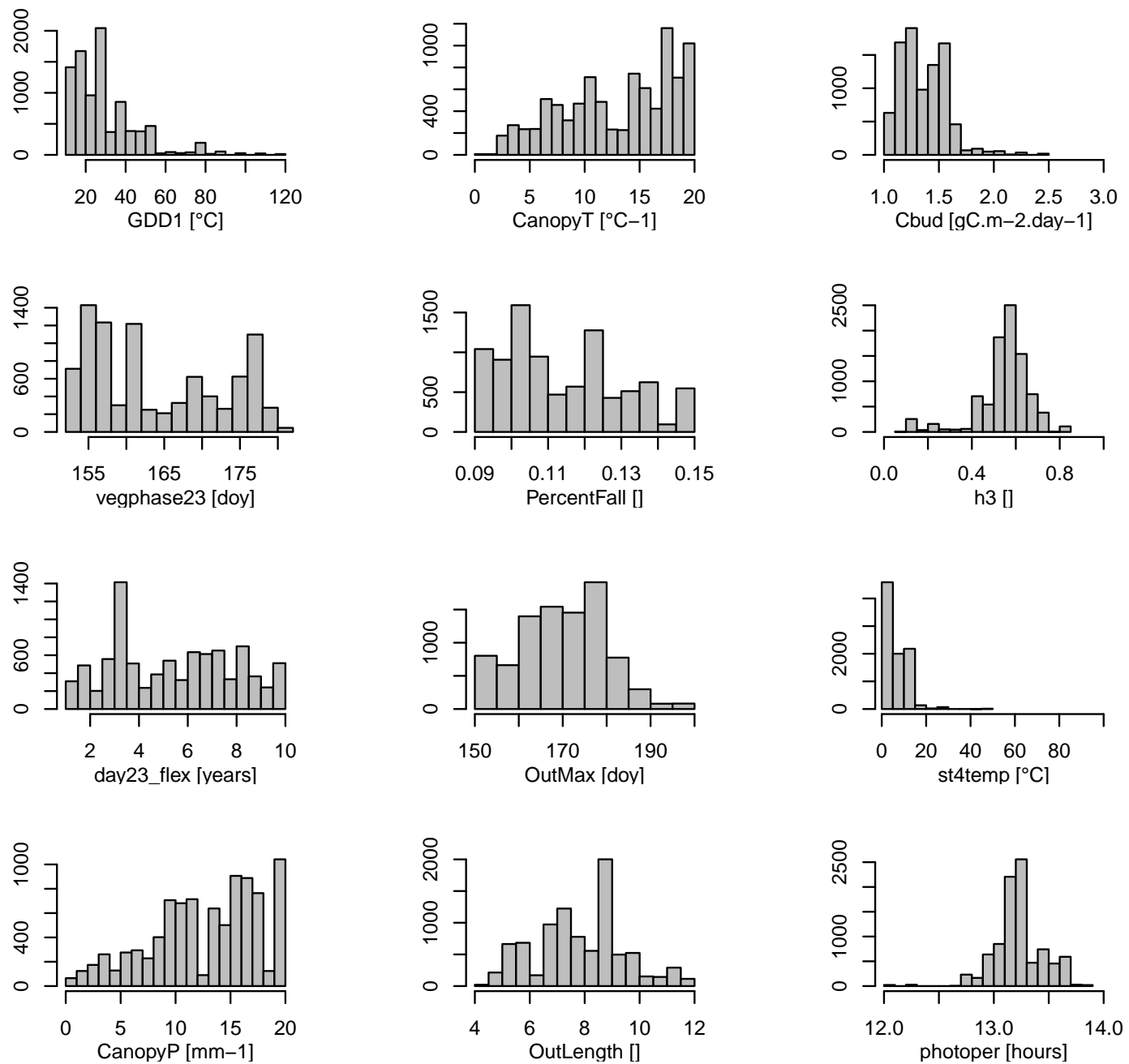

Figure S43. Posterior frequency distributions of carbon allocation parameters (Table S3) at WROZ site (NRCAN (5') climatic dataset) (Fig. 1b, Table 2) for the 1950-2000 calibration period. 

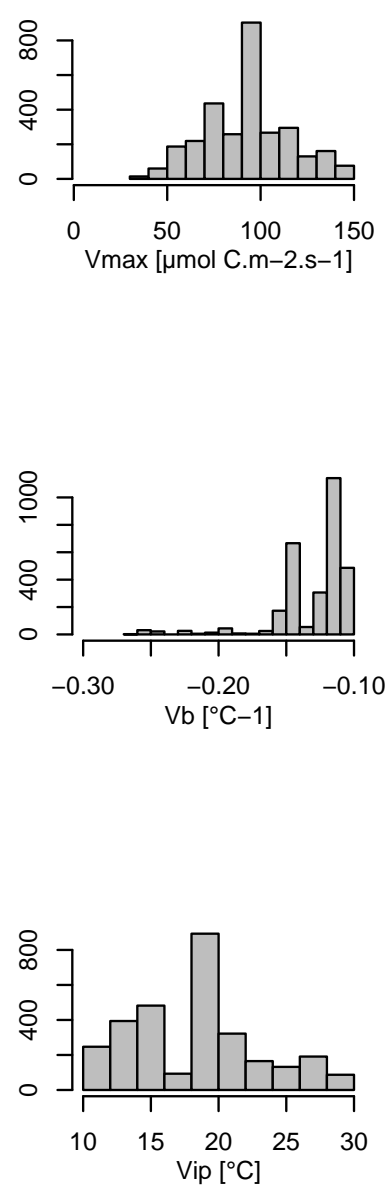
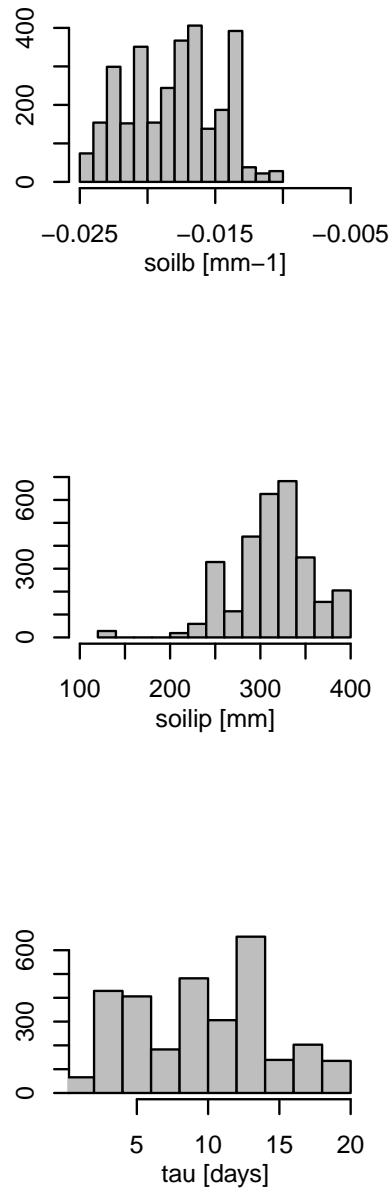

Figure S44. Posterior frequency distributions of photosynthesis parameters (Table S3) at WROZ site (NRCAN (5') climatic dataset) (Fig. 1b, Table 2) for the 1950-2000 calibration period. 


\section{Carbon allocation parameters for WH}
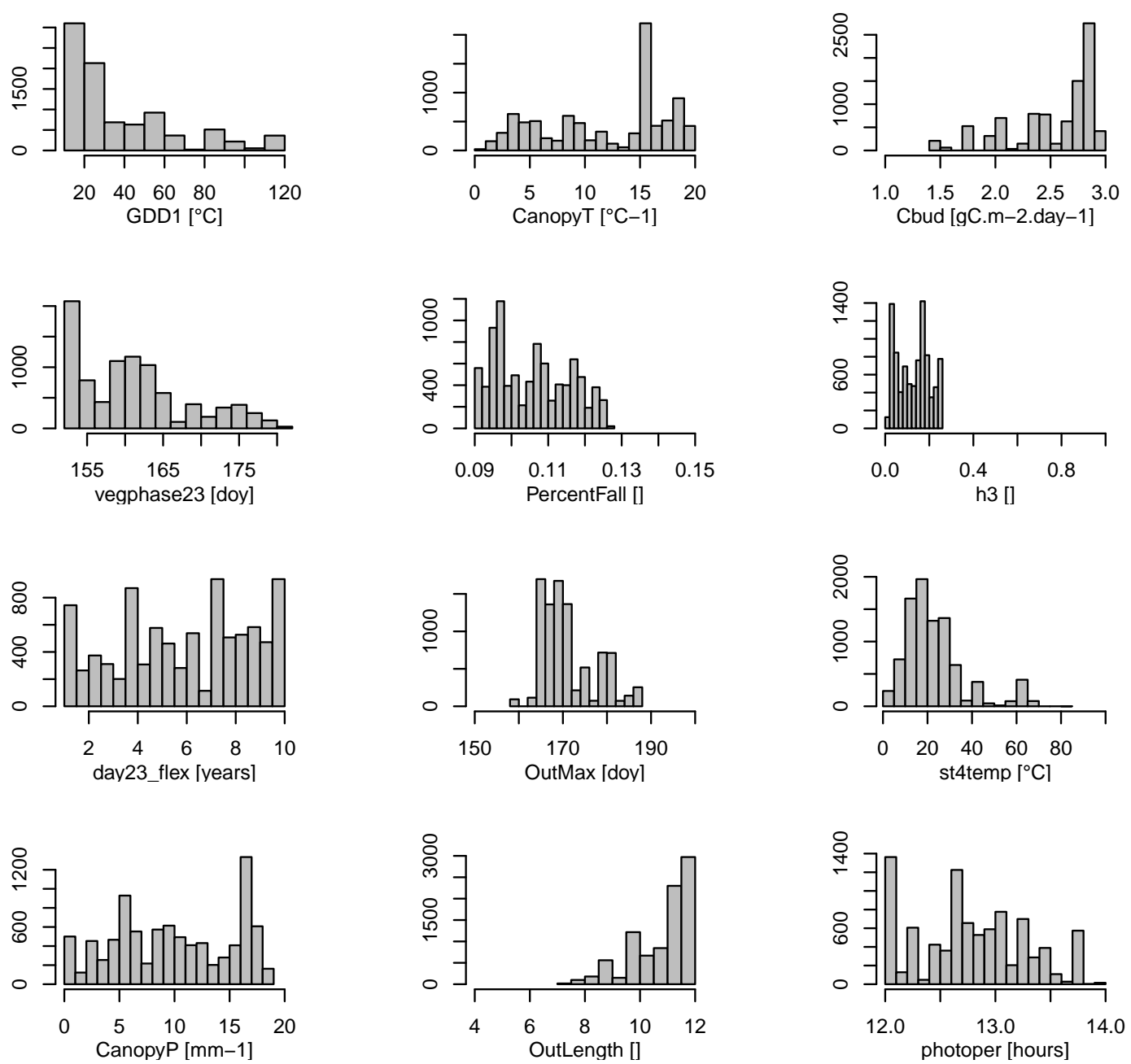

Figure S45. As in Fig. S43 at WH site. 


\section{Photosynthesis parameters for WH}
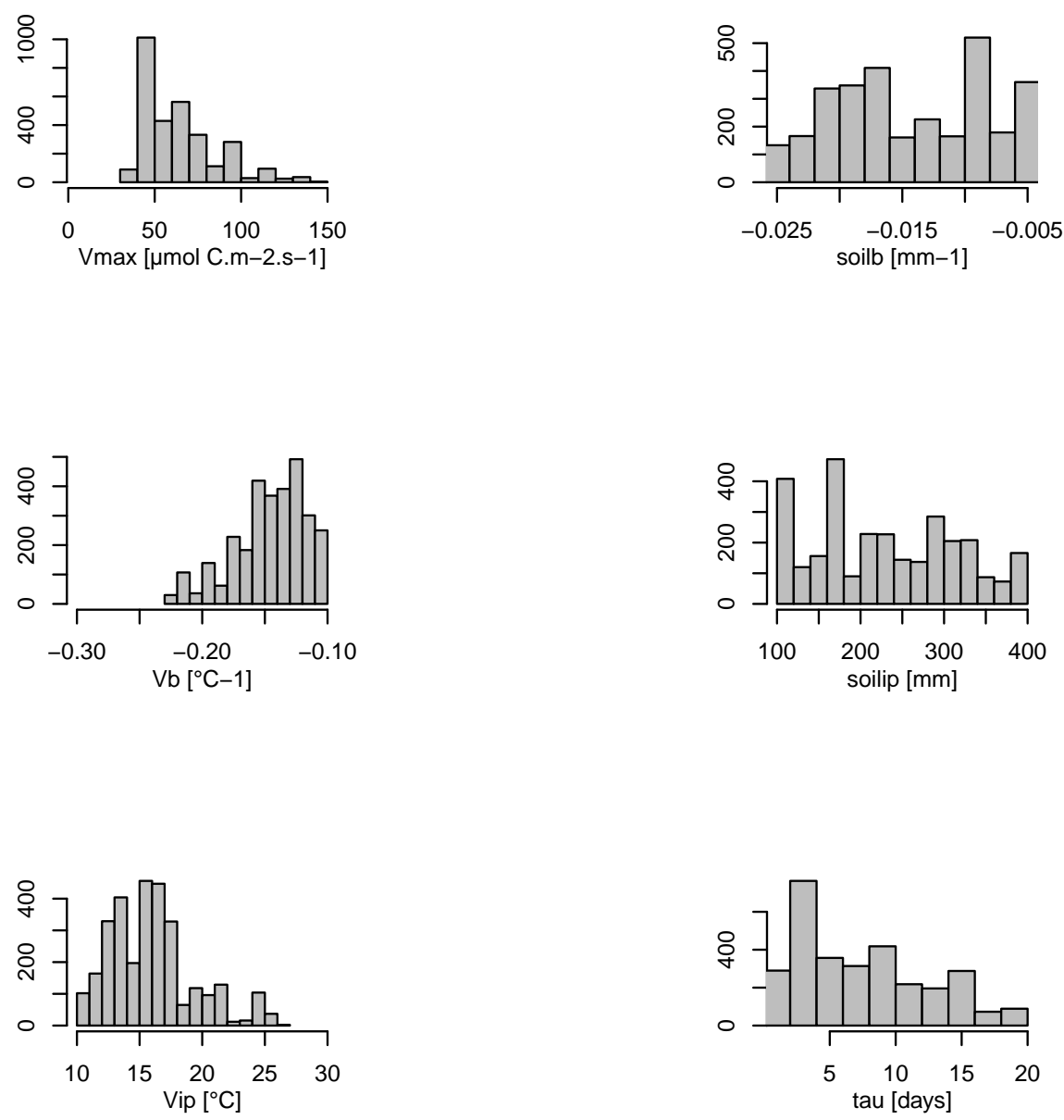

Figure S46. As in Fig. S44 at WH site. 


\section{Carbon allocation parameters for WNFL}
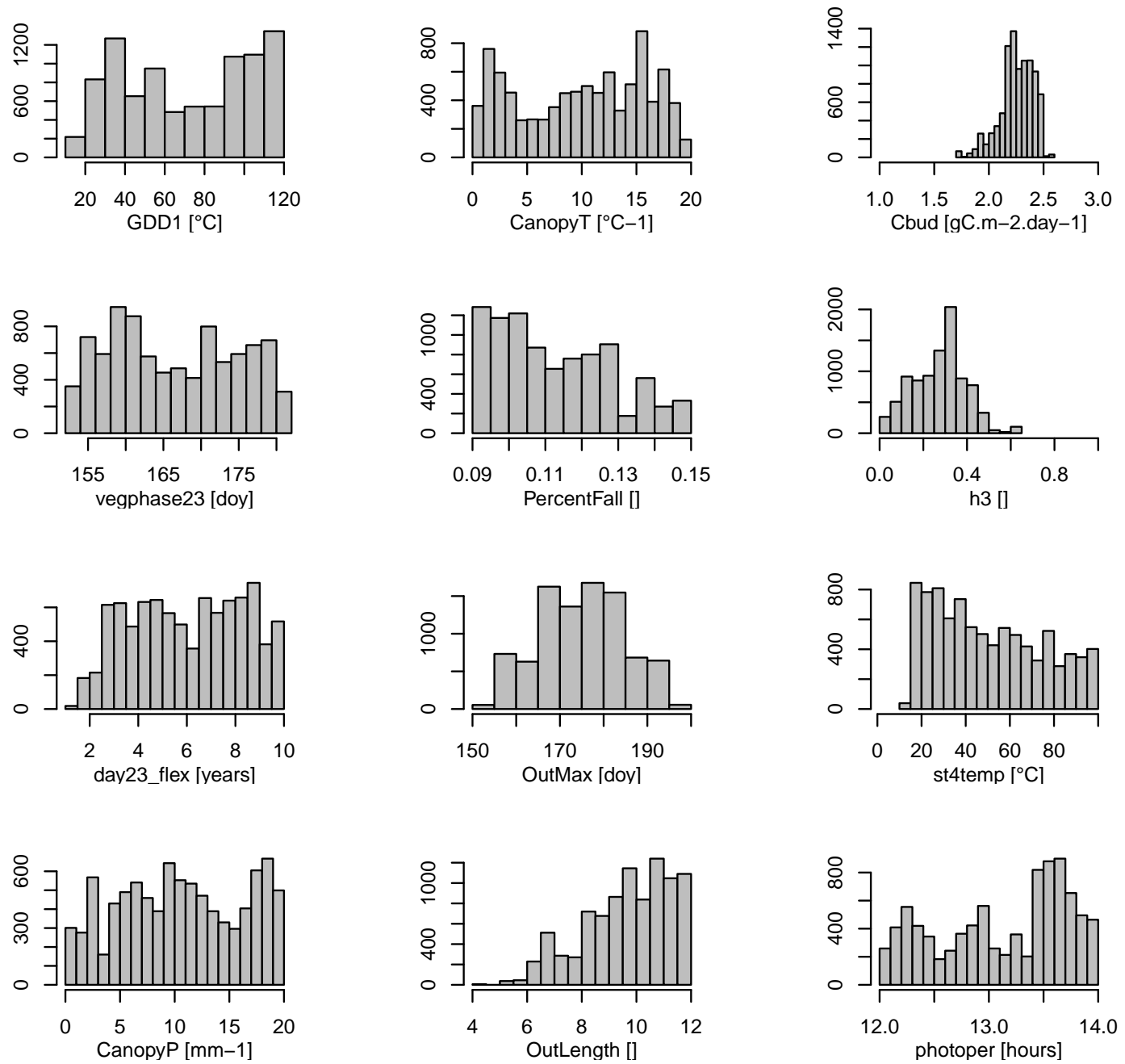

Figure S47. As in Fig. S43 at WNFL site. 
Photosynthesis parameters for WNFL
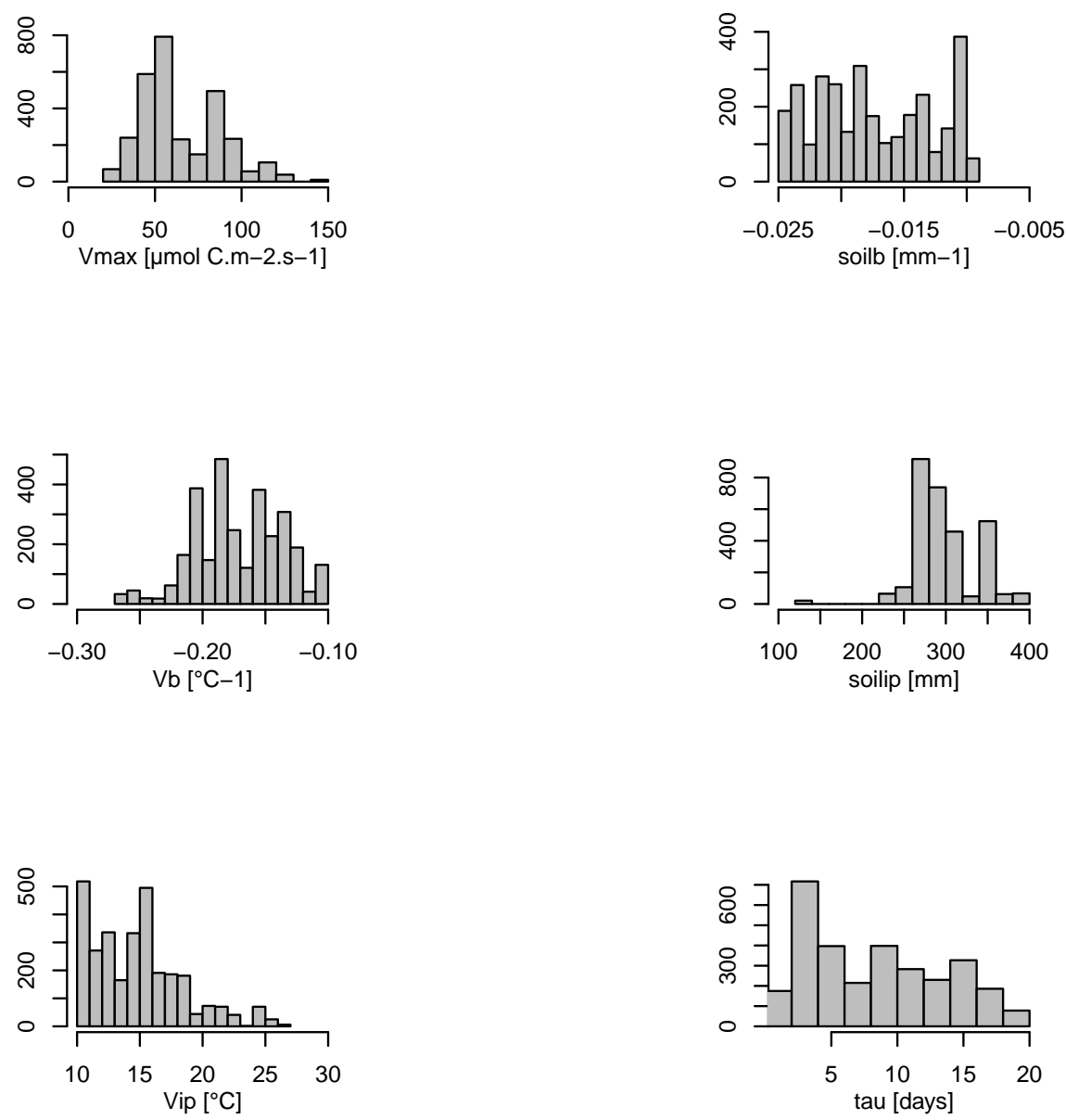

Figure S48. As in Fig. S44 at WNFL site. 


\section{Carbon allocation parameters for WCOR}
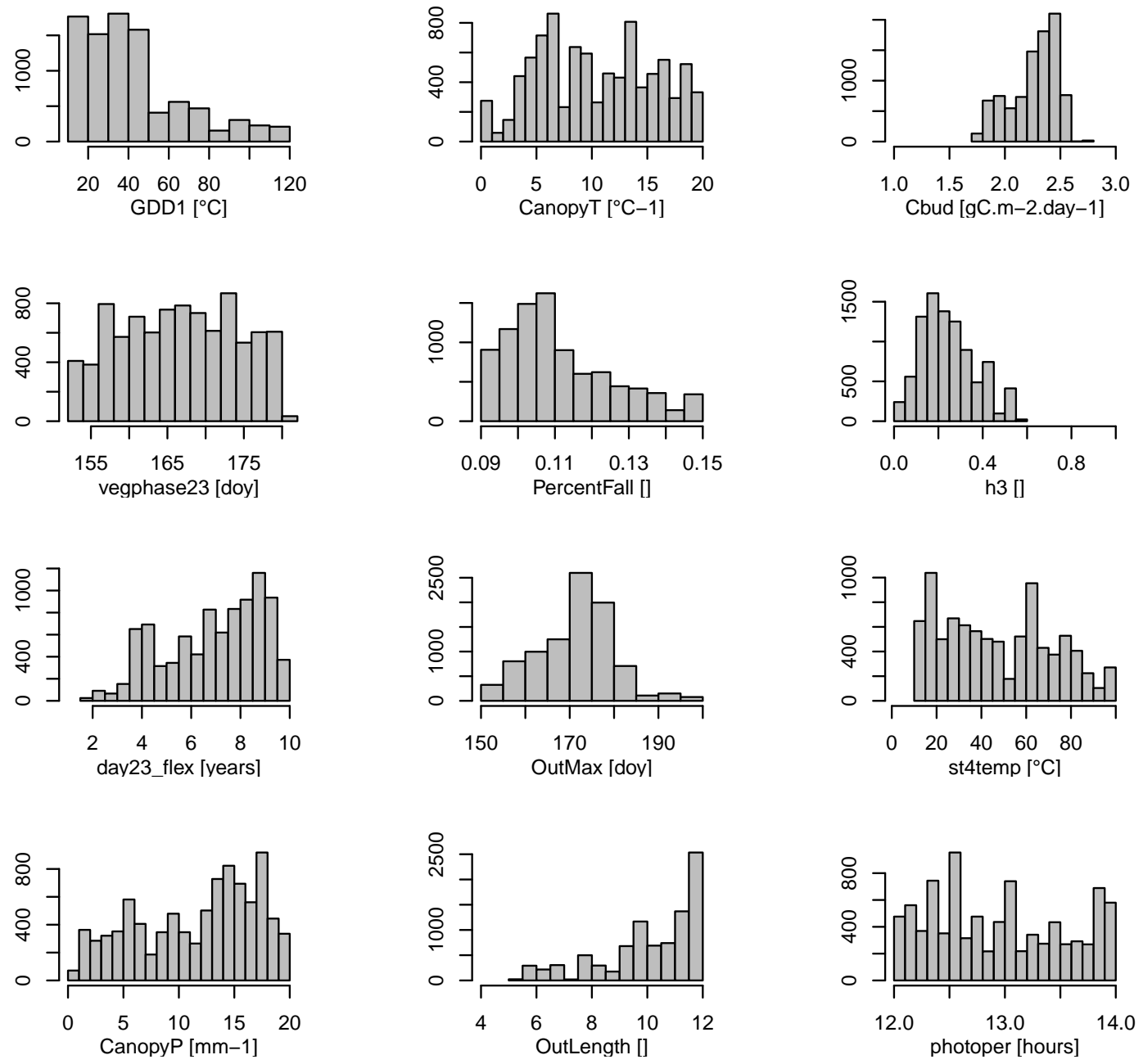

Figure S49. As in Fig. S43 at WCOR site. 

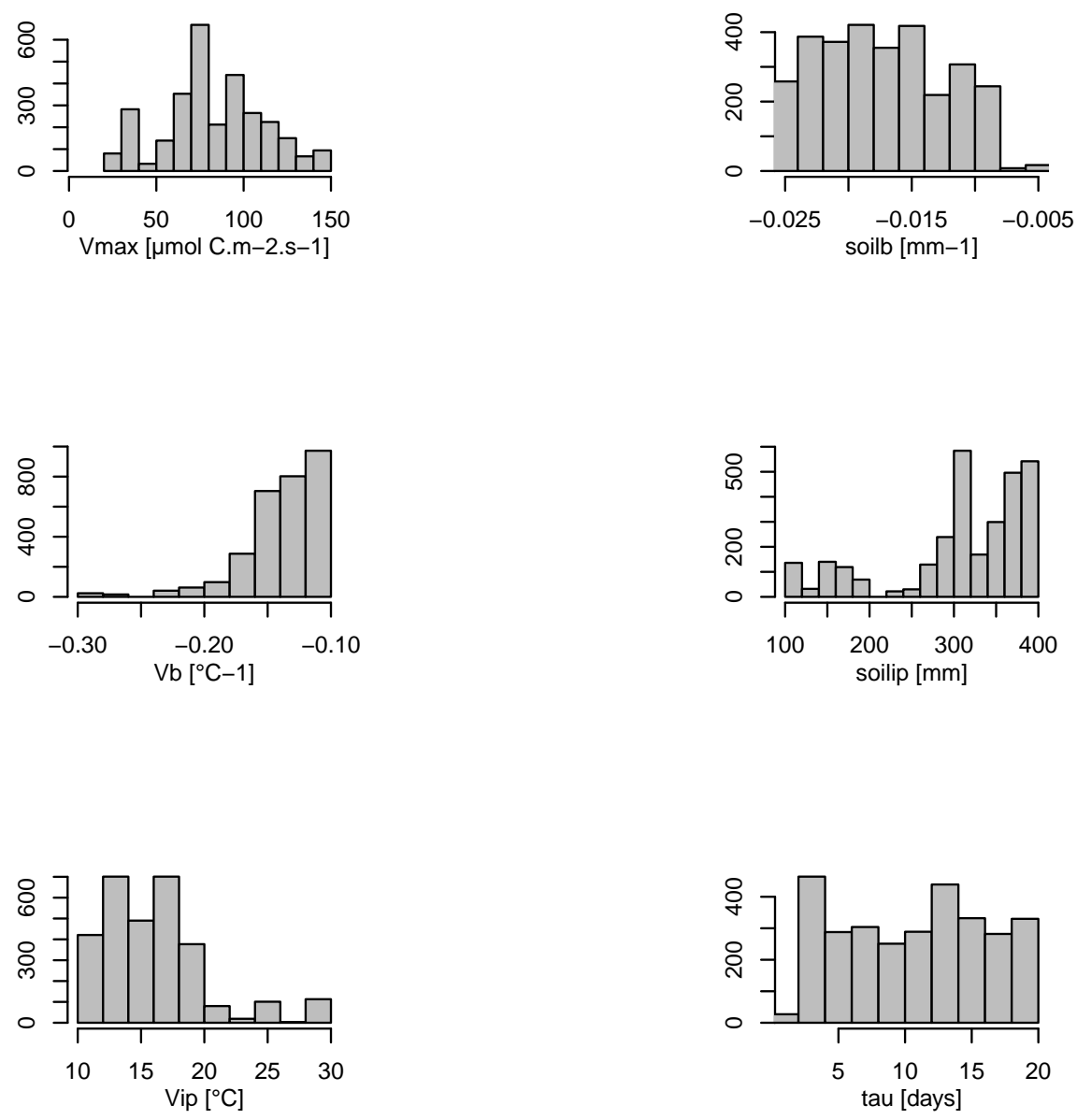

Figure S50. As in Fig. S44 at WCOR site. 


\section{Carbon allocation parameters for WDA1R_WTHH}
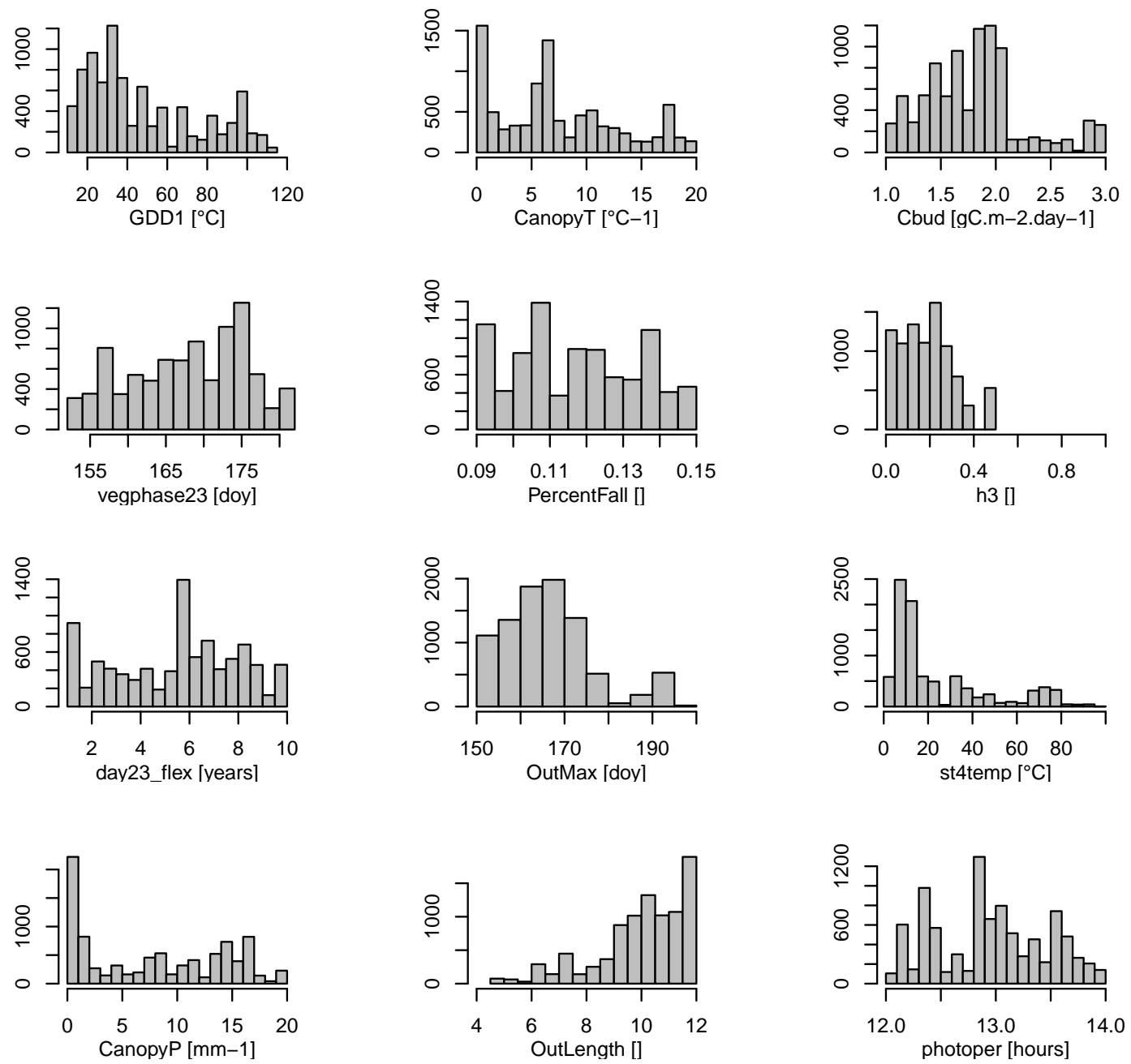

Figure S51. As in Fig. S43 at WDA1R_WTHH site. 

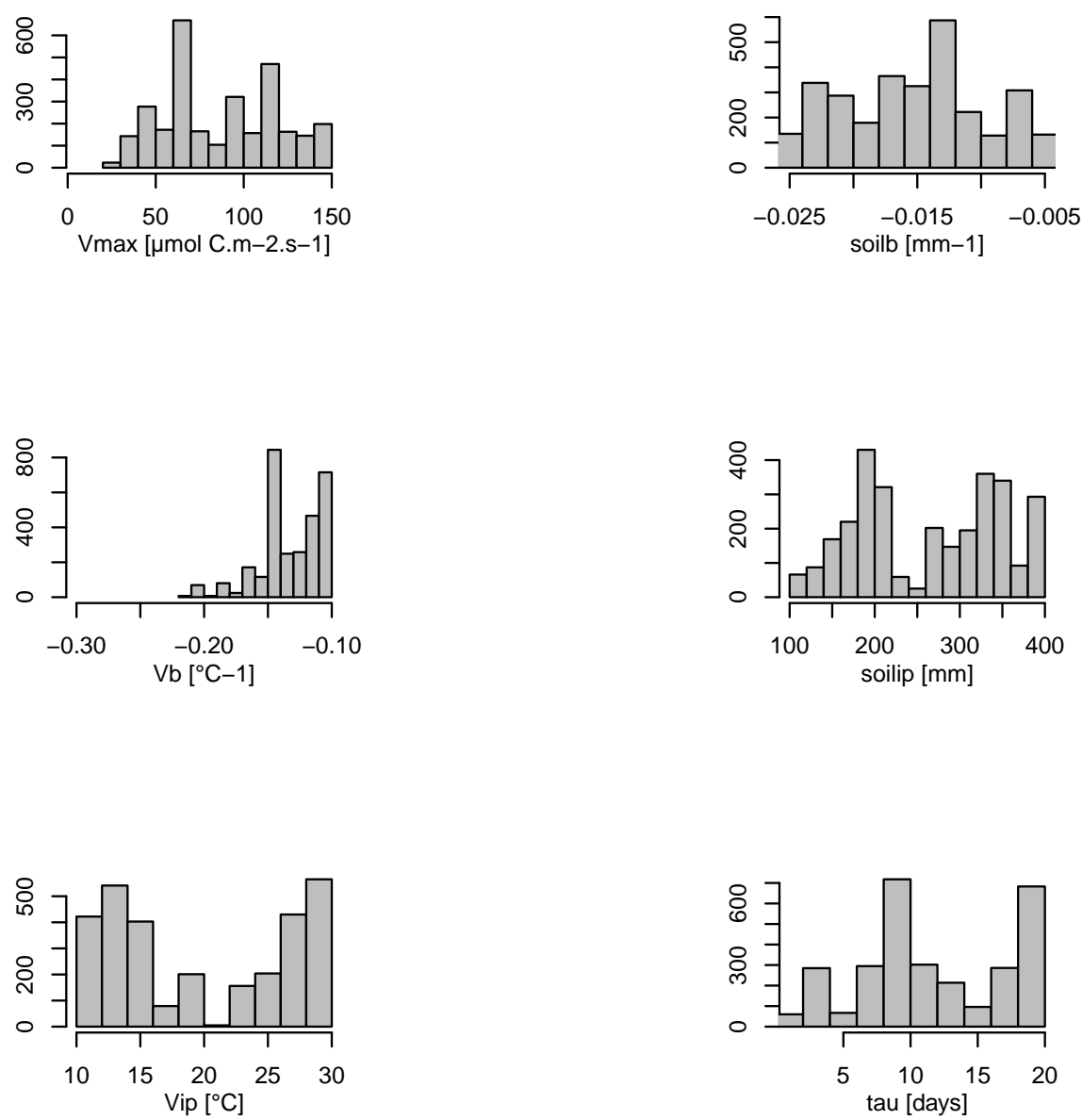

Figure S52. As in Fig. S44 at WDA1R_WTHH site. 


\section{Carbon allocation parameters for EALP}
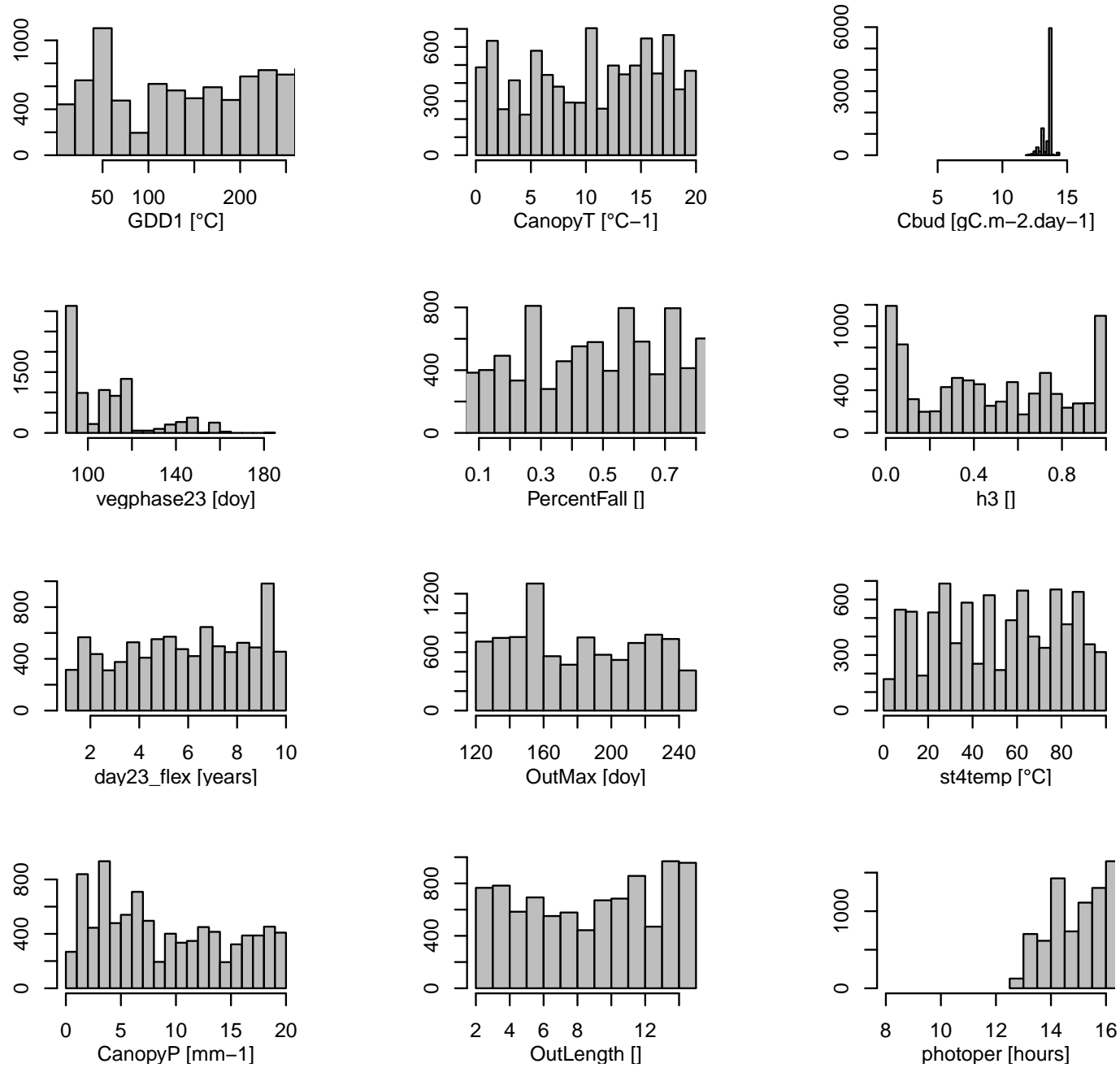

Figure S53. Posterior frequency distributions of carbon allocation parameters (Table S3) at EALP site (GHCN climate data) (Fig. 2, Table 2) for the 1950-2000 calibration period. 

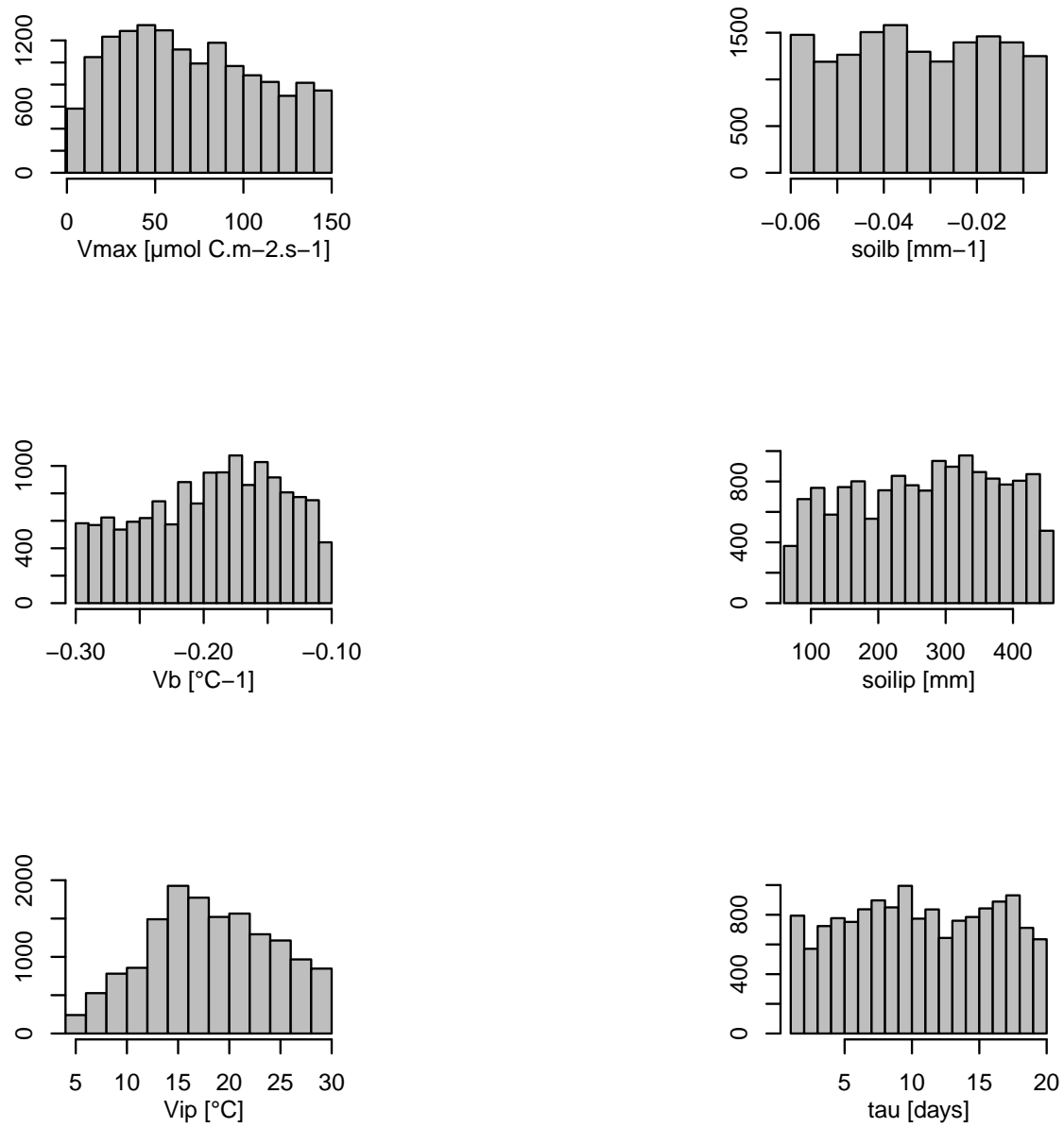

Figure S54. Posterior frequency distributions of photosynthesis parameters (Table S3) at EALP site (GHCN climate data) (Fig. 2, Table 2) for the 1950-2000 calibration period. 
Carbon allocation parameters for SWIT179
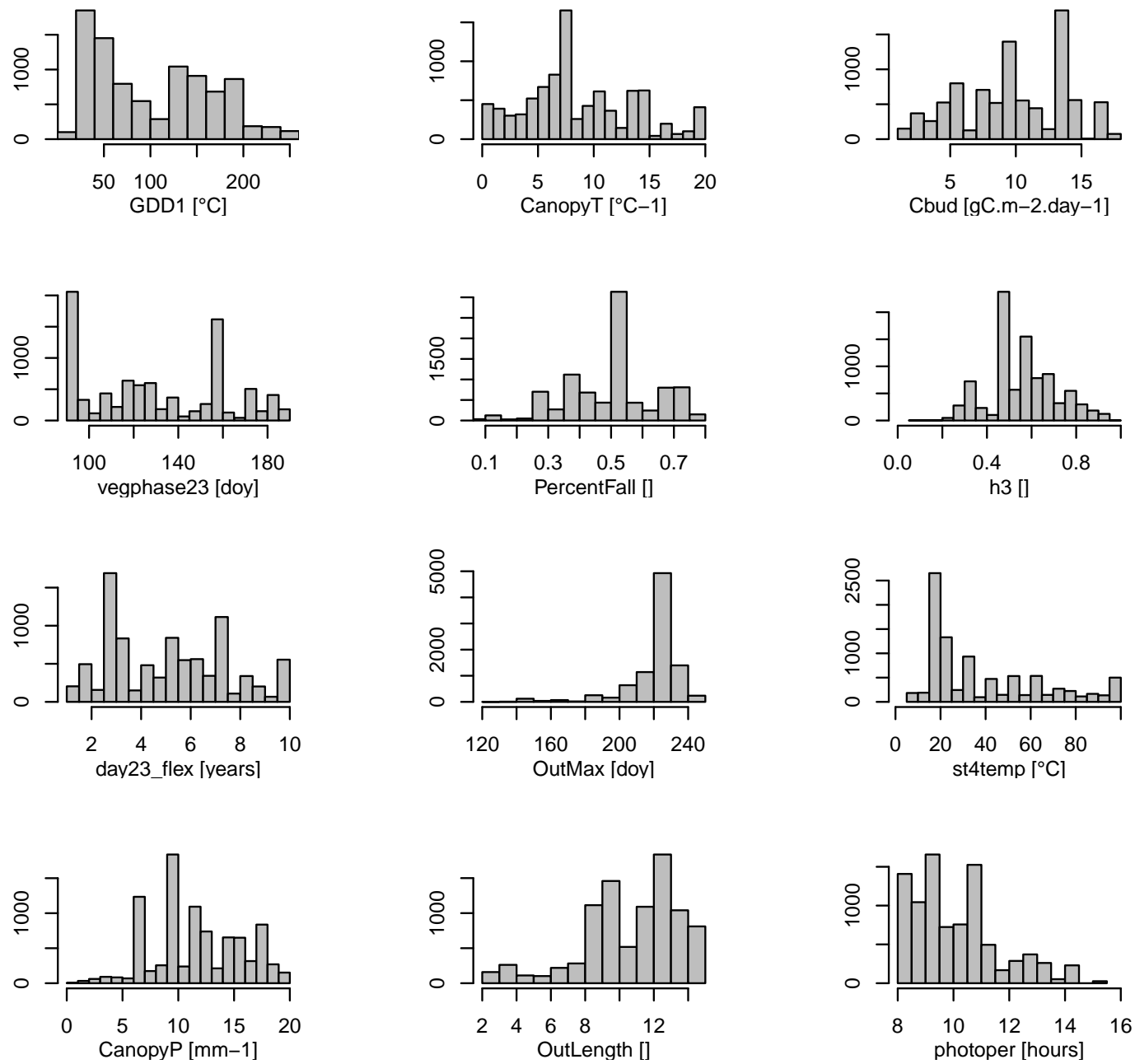

Figure S55. As in Fig. S53 at SWIT179 site. 

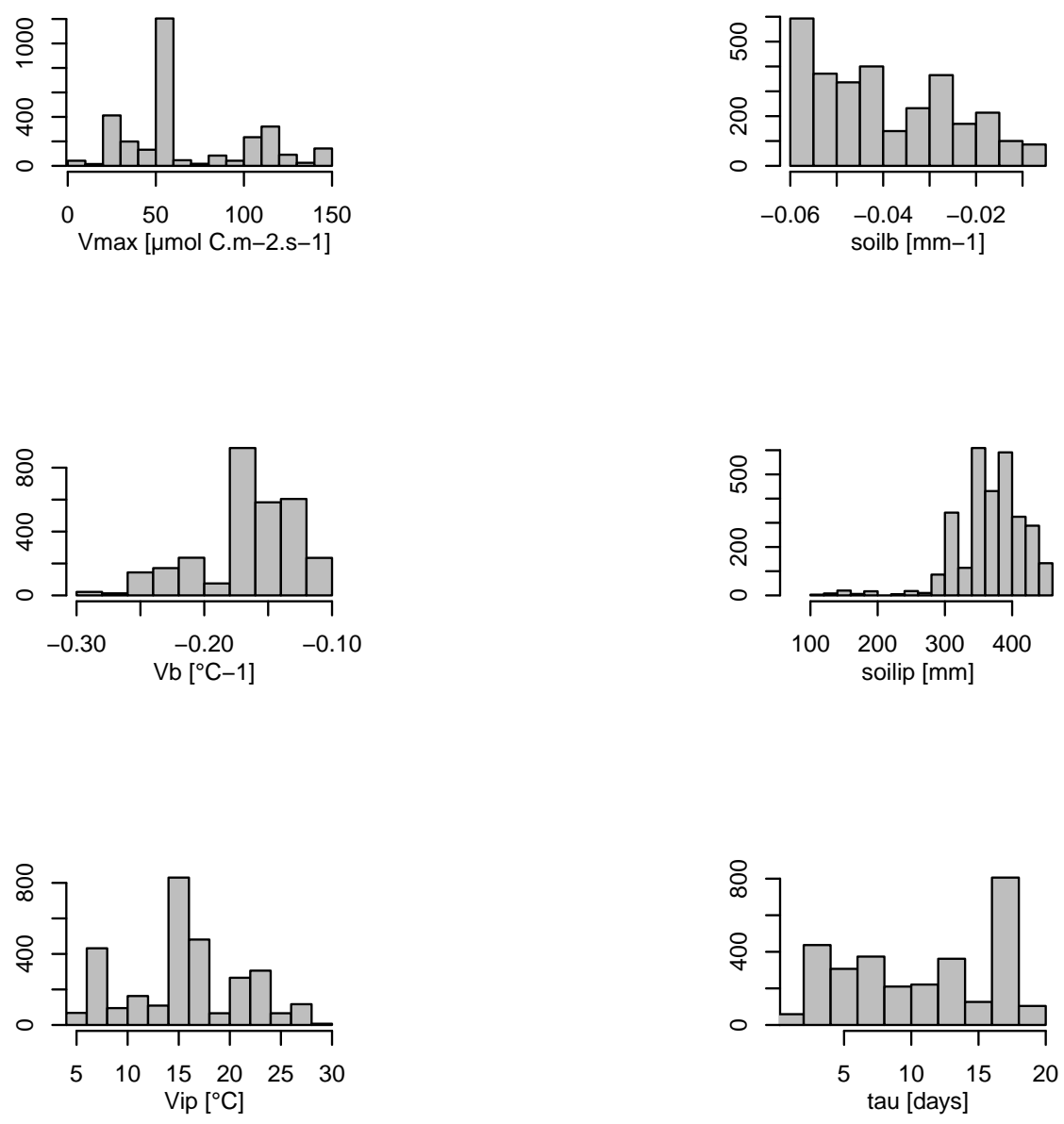

Figure S56. As in Fig. S54 at SWIT179 site. 


\section{Carbon allocation parameters for FINL045}
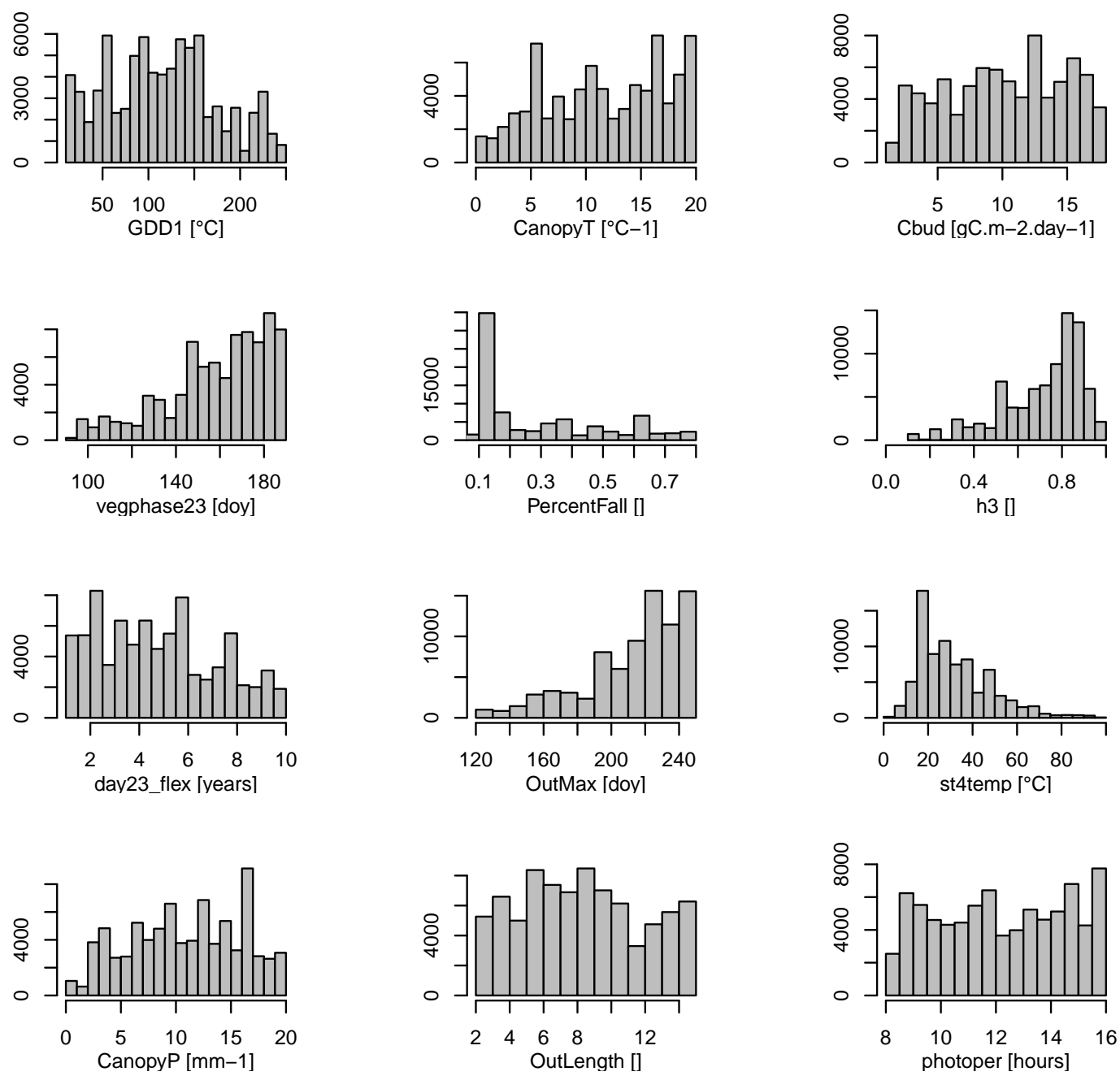

Figure S57. As in Fig. S53 at FINL045 site. 

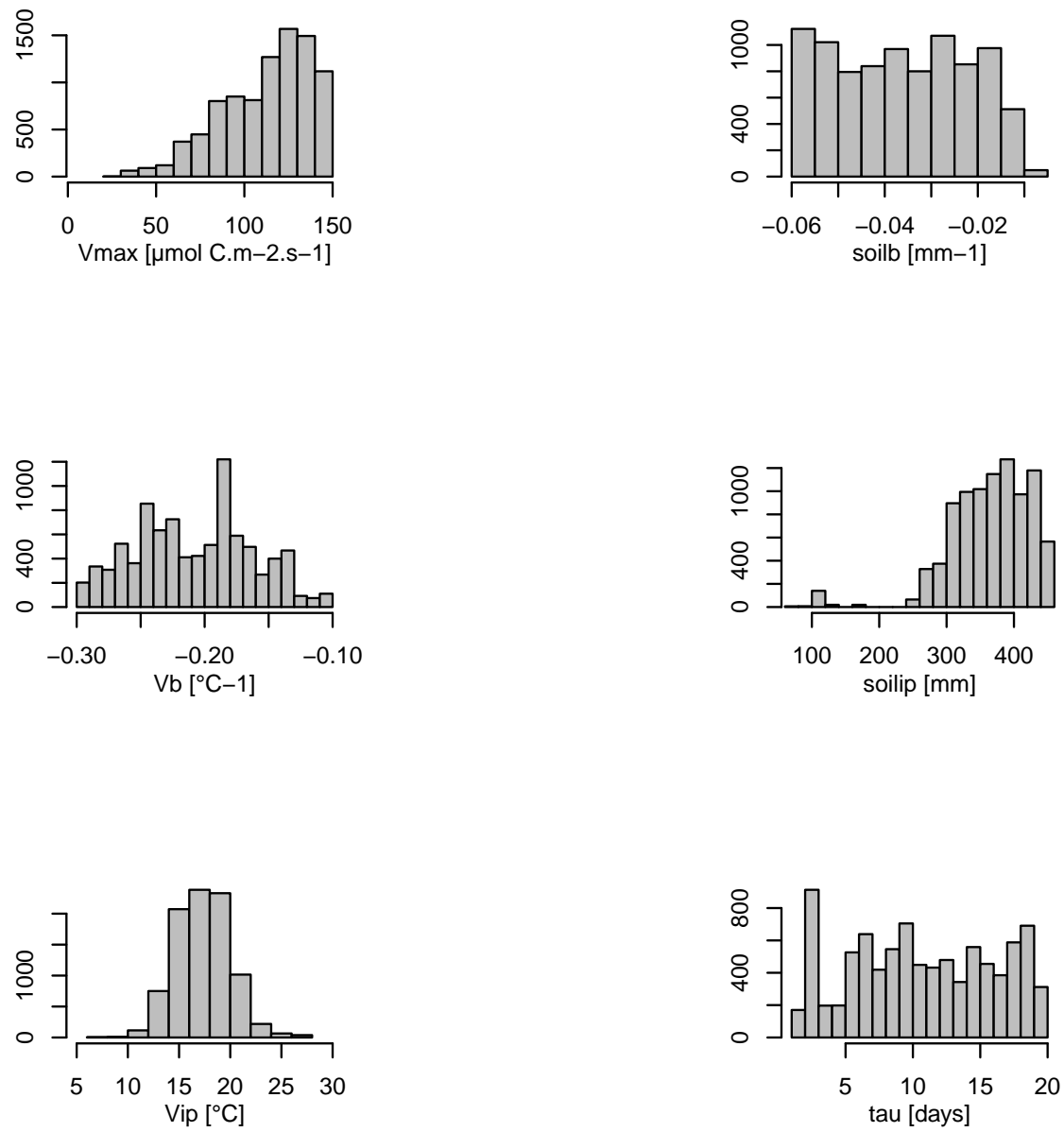

Figure S58. As in Fig. S54 at FINL045 site. 

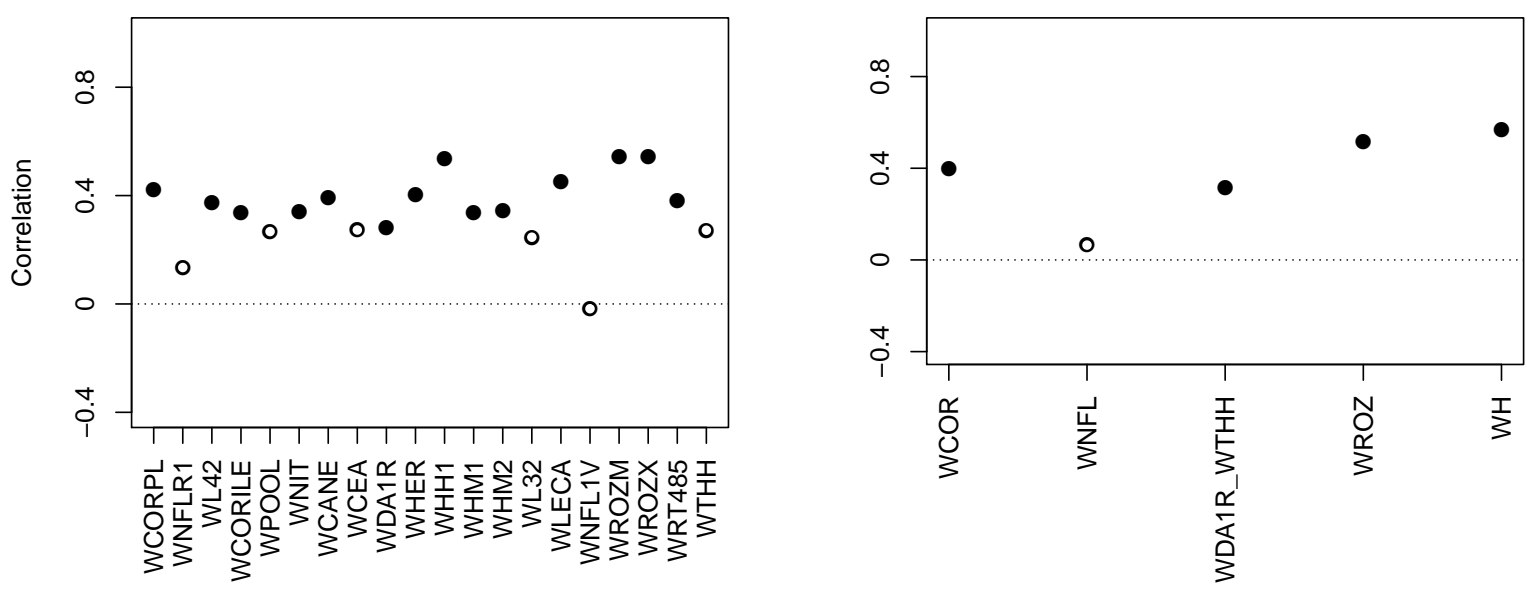

Figure S59. Pearson correlation coefficients between tree growth observations and simulations at the Eastern Canadian taiga sites (Fig. 1) with MAIDEN using NRCAN (5') as climatic inputs (Table 2) for the 1950-2000 period with $Q C \_t a i g a$ calibrated parameters from Gennaretti et al. (2017). Individual (left) and aggregated sites (right). The long-term decadal trends have been removed in observations and simulations. White inner circles stand for non-significant correlations ( $\mathrm{p}$-value $>0.05$ ). Plain circles stand for significant correlations ( $\mathrm{p}$-value $<0.05)$. 

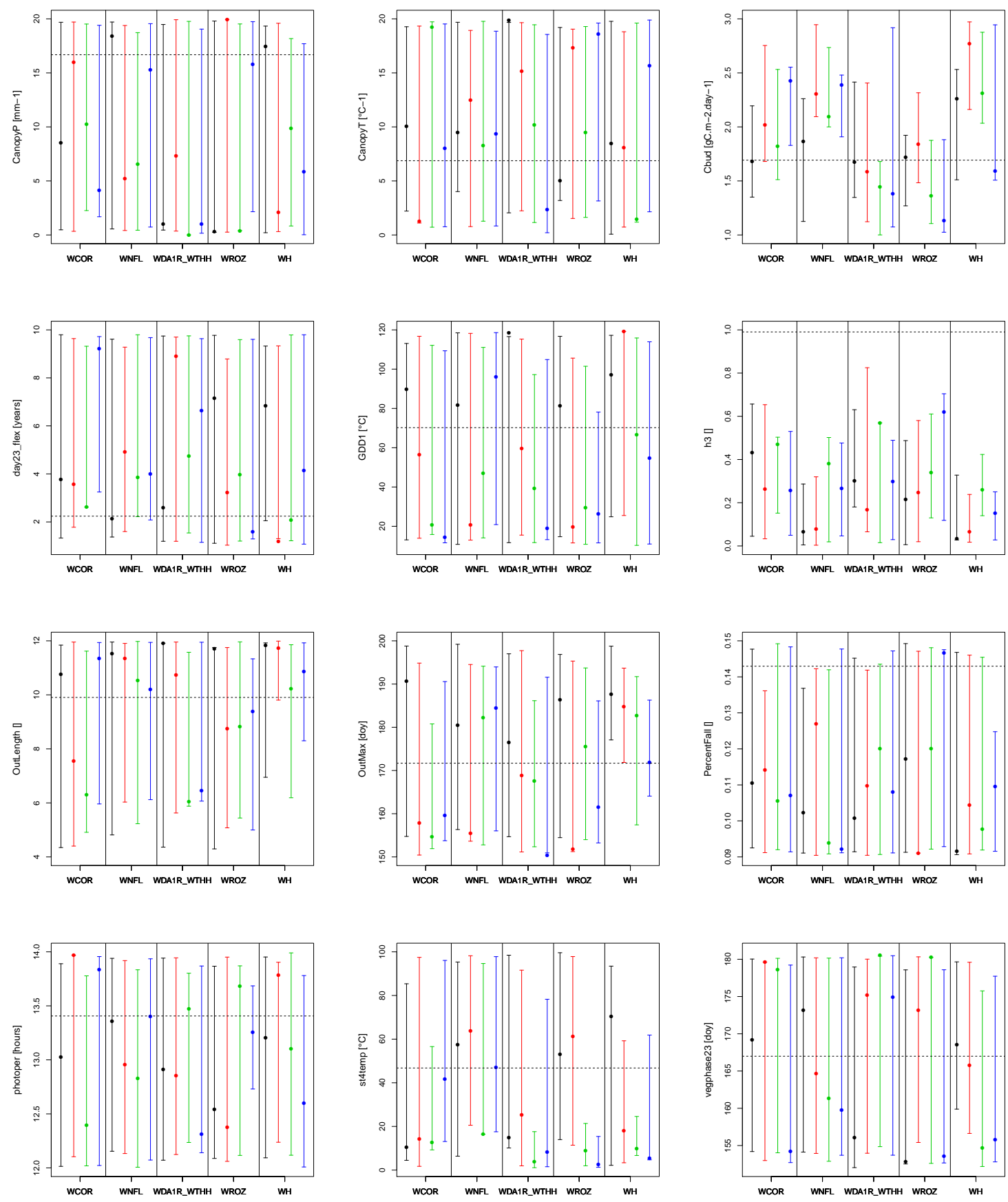

- 20CRv2c - 20CRv2c corr. - GMF - NRCAN

Figure S60. Selected carbon allocation parameters value (Table S3) based on the calibration procedure detailed in Sect. 2.3.1 and 95\% confidence interval of each parameter (computed based on all iterations of the third step of the calibration process, with a five iterations thinning and a burn-in period of 3000 iterations, see Sect. 2.3.1) for the five aggregated Eastern Canadian sites (Fig. 1b) and for all climatic datasets (Table 2) over the 1950-2000 time period. Dashed line corresponds to the parameter value at $Q C \_t a i g a$ site from Gennaretti et al. (2017). 

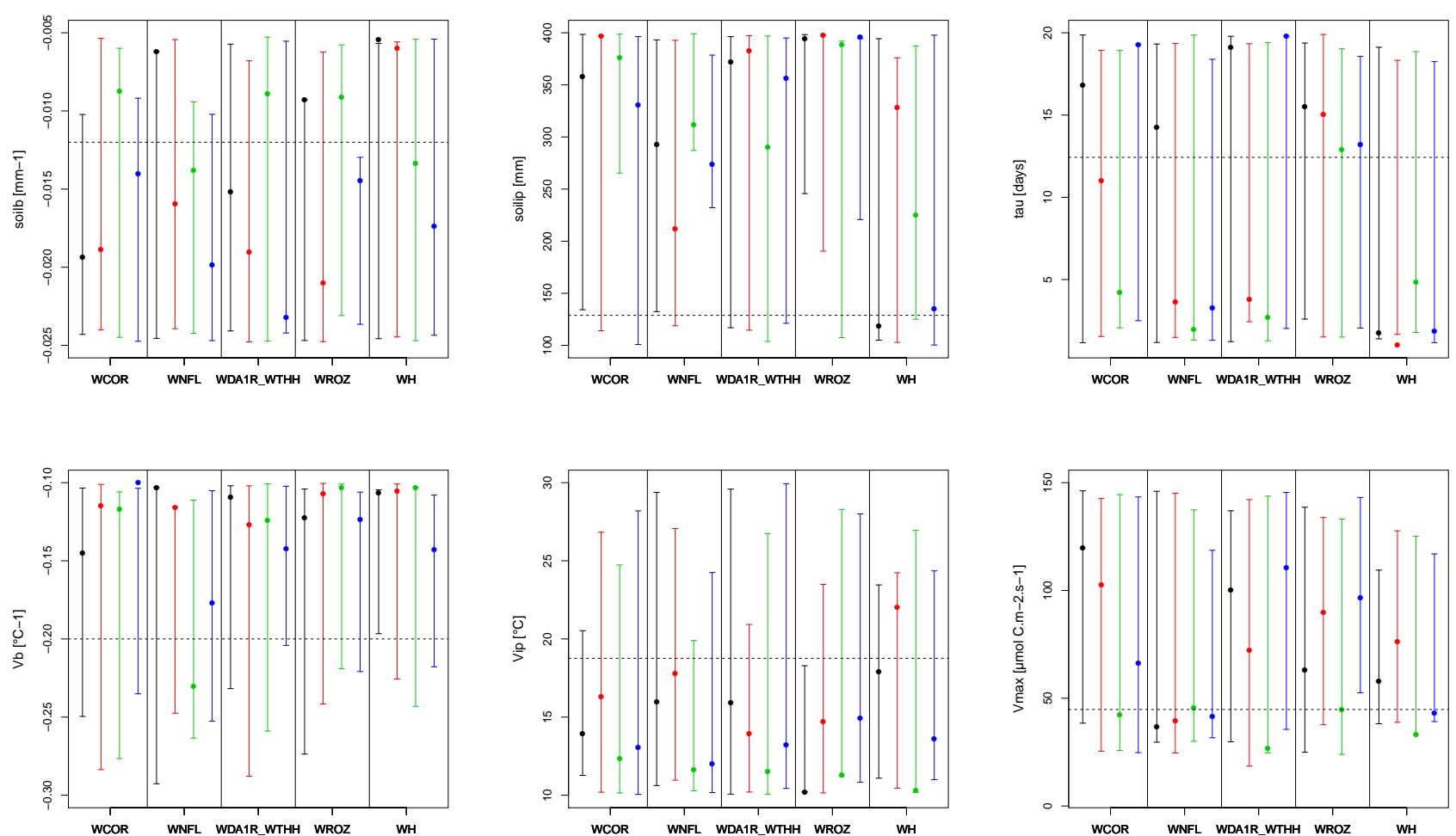

- 20CRv2c - 20CRv2c corr — GMF — NRCAN

Figure S61. Selected photosynthesis parameters value (Table S3) based on the calibration procedure detailed in Sect. 2.3 .1 and $95 \%$ confidence interval of each parameter (computed based on all iterations of the second step of the calibration process, with a five iterations thinning and a burn-in period of 1000 iterations, see Sect. 2.3.1) for the five aggregated Eastern Canadian sites (Fig. 1b) and for all climatic datasets (Table 2) over the 1950-2000 time period. Dashed line corresponds to the parameter value at QC_taiga site from Gennaretti et al. (2017). 

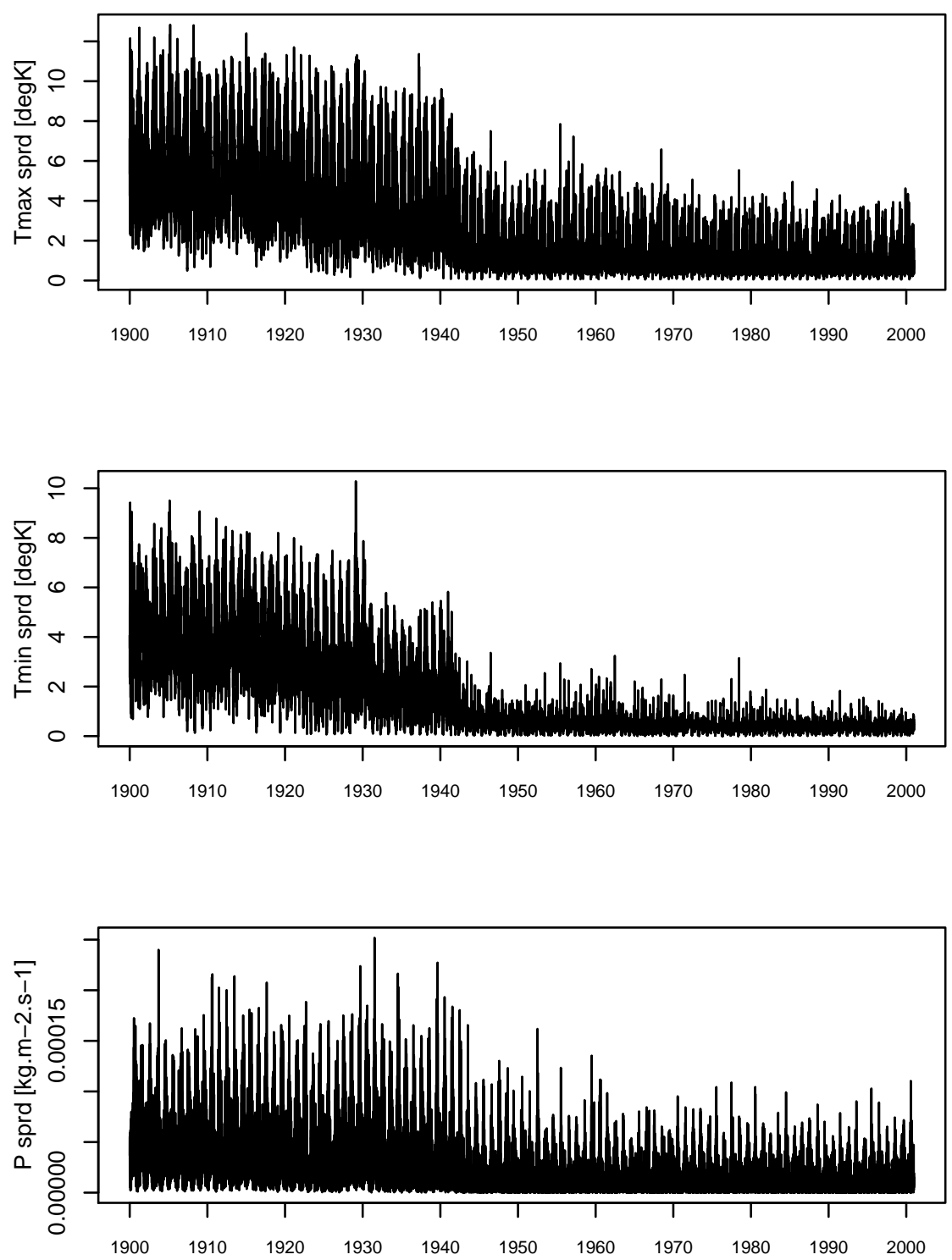

Figure S62. WL42 (Fig. 1a). Ensemble spread of maximum temperature (Tmax sprd), minimum temperature (Tmin sprd) and precipitations (P sprd) for the NOAA-CIRES 20th Century Reanalysis V2c (Table 2) for the 1900-2000 time period. 


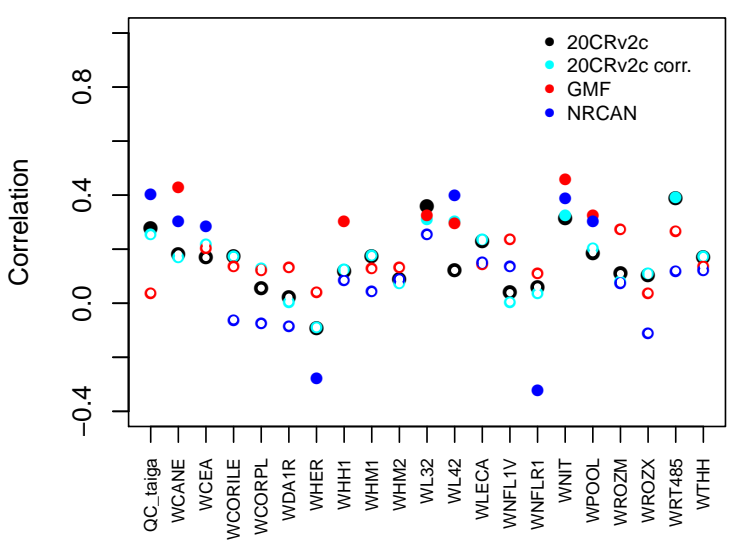

(a) $1950-2000$

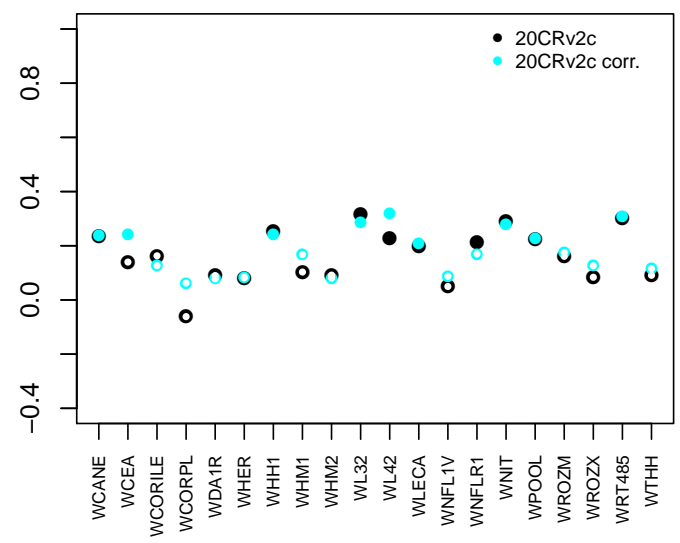

(b) $1900-2000$

Figure S63. Pearson correlation coefficients between tree growth observations and simulations at the Eastern Canadian taiga sites (Fig. 1a) with VS-Lite using the different climatic datasets described in Table 2 for the 1950-2000 (a) and 1900-2000 (b) calibration periods. White inner circles stand for non-significant correlations ( $\mathrm{p}$-value $>0.05$ ). 